KEK-TH-1084

OIQP-06-06

May 2006

\title{
Non-Relativistic AdS Branes and Newton-Hooke Superalgebra
}

\author{
Makoto Sakaguchi $^{a}$ and Kentaroh Yoshida ${ }^{b}$ \\ a Okayama Institute for Quantum Physics \\ Kyoyama 1-9-1, Okayama 700-0015, Japan \\ makoto_sakaguchi@pref .okayama.jp \\ ${ }^{b}$ Theory Division, Institute of Particle and Nuclear Studies, \\ High Energy Accelerator Research Organization (KEK), \\ Tsukuba, Ibaraki 305-0801, Japan. \\ kyoshida@post.kek.jp
}

\begin{abstract}
We examine a non-relativistic limit of D-branes in $\mathrm{AdS}_{5} \times \mathrm{S}^{5}$ and M-branes in $\mathrm{AdS}_{4 / 7} \times \mathrm{S}^{7 / 4}$. First, Newton-Hooke superalgebras for the AdS branes are derived from $\mathrm{AdS} \times \mathrm{S}$ superalgebras as Inönü-Wigner contractions. It is shown that the directions along which the AdS-brane worldvolume extends are restricted by requiring that the isometry on the AdS-brane worldvolume and the Lorentz symmetry in the transverse space naturally extend to the super-isometry. We also derive NewtonHooke superalgebras for pp-wave branes and show that the directions along which a brane worldvolume extends are restricted. Then the Wess-Zumino terms of the AdS branes are derived by using the Chevalley-Eilenberg cohomology on the super$\mathrm{AdS} \times \mathrm{S}$ algebra, and the non-relativistic limit of the AdS-brane actions is considered. We show that the consistent limit is possible for the following branes: $\mathrm{D} p$ (even,even) for $p=1 \bmod 4$ and $\mathrm{D} p$ (odd,odd) for $p=3 \bmod 4$ in $\mathrm{AdS}_{5} \times \mathrm{S}^{5}$, and M2 (0,3), M2 $(2,1)$, M5 $(1,5)$ and M5 $(3,3)$ in $\mathrm{AdS}_{4} \times \mathrm{S}^{7}$ and $\mathrm{S}^{4} \times \mathrm{AdS}_{7}$. We furthermore present non-relativistic actions for the AdS branes.
\end{abstract}




\section{Introduction}

The AdS/CFT conjecture [1] predicts that type IIB superstring theory in $\mathrm{AdS}_{5} \times \mathrm{S}^{5}$ is dual to the four-dimensional $\mathcal{N}=4 \mathrm{SU}(N)$ super Yang-Mills theory in large $N$ limit. Though it is too hard to analyze the full AdS superstring, Berenstein-Maldacena-Nastase (BMN) found a nice way to extract a solvable subsector (referred to as BMN sector) [2]. Taking this subsector corresponds to the so-called Penrose limit for the AdS geometry [3], and the relevant symmetry to the BMN sector is the pp-wave superalgebra, which is obtained as an Inönü-Wigner (IW) contraction [4] of the super- $\mathrm{AdS}_{5} \times \mathrm{S}^{5}$ algebra [5] (see [6] for the eleven-dimensional cases).

A non-relativistic limit of strings in flat spacetime provides another solvable sector [7] (see also [8]). This limit is a truncation of the full theory in the sense that light states satisfying a Galilean invariant dispersion relation are kept and the rest decouples. The relevant symmetry is the Galilean limit of the Poincaré algebra. The non-relativistic flat branes are examined in [9-13]. In $[14,15]$ these studies have been extended to branes in AdS spaces. In particular a Lorentzian F-string in $\mathrm{AdS}_{5} \times \mathrm{S}^{5}$, i.e. $\mathrm{AdS}_{2}$ brane, was examined in [15]. They showed that the F-string theory in $\operatorname{AdS}_{5} \times \mathrm{S}^{5}$ is reduced to a free theory in the non-relativistic limit, and so the resulting theory is exactly solvable. In the non-relativistic limit, the super- $\mathrm{AdS}_{5} \times \mathrm{S}^{5}$ algebra is also contracted to the Newton-Hooke $(\mathrm{NH})$ superalgebra for the F-string. Then the isometry of the $\mathrm{AdS}_{2}$-brane worldvolume, the $\mathrm{AdS}_{2}$ algebra so(1,2), and the Lorentz symmetry in the transverse space, so(3)×so(5), extend to a super-isometry algebra.

In this paper we consider D-branes in $\mathrm{AdS}_{5} \times \mathrm{S}^{5}$ and M-branes in $\mathrm{AdS}_{4 / 7} \times \mathrm{S}^{7 / 4}$. First we examine D-branes in $\mathrm{AdS}_{5} \times \mathrm{S}^{5}$. In addition to $\mathrm{AdS}_{2}$ brane, there exist various AdS branes in $\mathrm{AdS}_{5} \times \mathrm{S}^{5},(m, n)$ branes of which worldvolume extends along $m$ directions in $\mathrm{AdS}_{5}$ and $n$-directions in $\mathrm{S}^{5}$. In our previous works [16-19], we have classified some possible configurations of the D-branes in $\mathrm{AdS}_{5} \times \mathrm{S}^{5}$ by examining the $\kappa$-variation surface terms of an open superstring. Here we will classify possible configurations of D-branes by requiring that the isometry of the AdS brane worldvolume $\operatorname{AdS}_{m} \times \mathrm{S}^{n}\left(\mathrm{H}^{m} \times \mathrm{S}^{n}\right)$ and

the Lorentz symmetry in the transverse space $\mathbb{E}^{5-m} \times \mathbb{E}^{5-n}\left(\mathbb{E}^{4-m, 1} \times \mathbb{E}^{5-n}\right)$, i.e., so $(m-$ $1,2) \times \mathrm{so}(n+1) \times \mathrm{so}(5-m) \times \mathrm{so}(5-n)$ for a Lorentzian brane and $\mathrm{so}(m, 1) \times \mathrm{so}(n+1) \times \mathrm{so}(4-$ $m, 1) \times \operatorname{so}(5-n)$ for a Euclidean brane, naturally extend to the super-isometry. The result surely contains our previous result, but some new configurations are allowed to exist. 
We furthermore derive the NH superalgebras for these branes as IW contractions of the super- $\mathrm{AdS}_{5} \times \mathrm{S}^{5}$ algebra. The similar analyses are applied to branes in IIB pp-wave, and derive the NH superalgebras for these branes as IW contractions of the IIB pp-wave superalgebra.

The Wess-Zumino (WZ) terms for $p$-branes in flat spacetime can be classified [20] as non-trivial elements of the Chevalley-Eilenberg (CE) cohomology [21]. This is generalized to D-branes in $[22,23]$ by introducing an additional two form which corresponds to a modified field strength of the background $B$ field. Here we examine the WZ terms for AdS branes by using the CE cohomology on $\mathfrak{g}$ of the supergroup

$$
G=\operatorname{PSU}(2,2 \mid 4) /(\mathrm{SO}(4,1) \times \mathrm{SO}(5)), \quad \text { i.e. "super- } \mathrm{AdS}_{5} \times \mathrm{S}^{5} \text { " / "Lorentz" . }
$$

We show that the WZ terms of AdS branes can be classified as non-trivial elements of the CE cohomology, except for the WZ term of a string which is a trivial element [24,25].

Expanding the supercurrents with respect to the scaling used in the IW contraction, we obtain the non-relativistic limit of the brane action. In comparison to the Penrose limit in which the leading terms in the expansion contribute to the pp-wave brane actions (see Appendix C), in the non-relativistic limit the leading order terms of the Dirac-Born-Infeld (Nambu-Goto) part and the WZ part cancel out each other, and the next-to-leading order terms contribute to the non-relativistic action. We find that the consistent non-relativistic limit exists only for $\mathrm{D} p$ (even, even) for $p=1 \bmod 4$ and $\mathrm{D} p$ (odd, odd) for $p=3 \bmod 4$ in $\mathrm{AdS}_{5} \times \mathrm{S}^{5}$. We derive the non-relativistic AdS D-brane action and find that it is reduced to a simple action by fixing the $\kappa$-gauge symmetry and the worldvolume reparametrization. While the non-relativistic AdS D-string action is a free field action, the non-relativistic AdS D $p$-brane action $(p>1)$ contains an additional term which originates from the flux contribution in the WZ term. The non-relativistic flat D-brane actions obtained in [12] are reproduced as a flat limit of the non-relativistic AdS D-brane actions.

Next we examine a non-relativistic limit of M-branes in $\mathrm{AdS}_{4 / 7} \times \mathrm{S}^{7 / 4}$. The $\mathrm{NH}$ superalgebra for M-branes are derived as IW contractions of the super- $\mathrm{AdS}_{4 / 7} \times \mathrm{S}^{7 / 4}$ algebras. To achieve this, we show that the directions along which a brane worldvolume extends are restricted by requiring that the isometry of the AdS brane worldvolume and the Lorentz symmetry in the transverse space naturally extend to the super-isometry, and that possible M-branes are classified. As expected, the configurations obtained in [26,27] by examining the $\kappa$-variation surface term of an open supermembrane are contained in the 
above classification. The similar analyses are applied to branes in $\mathrm{M}$ pp-wave, and derive the NH superalgebras for these branes as IW contractions of the M pp-wave superalgebra. We obtain the WZ terms of AdS branes as non-trivial elements of the CE cohomology on $\mathfrak{g}$ of the supergroup

$$
G=\mathrm{OSp}(8 \mid 4) /(\mathrm{SO}(3,1) \times \mathrm{SO}(7)) \text { or } \mathrm{OSp}\left(8^{*} \mid 4\right) /(\mathrm{SO}(4) \times \mathrm{SO}(6,1)) \text {. }
$$

We find that the non-relativistic limit exists for M2 (0,3), M2 (2,1), M5 (1,5) and M5 $(3,3)$ in $\mathrm{AdS}_{4} \times \mathrm{S}^{7}$ and $\mathrm{S}^{4} \times \mathrm{AdS}_{7}$. By taking the non-relativistic limit of these AdS brane actions, we derive the non-relativistic M-brane actions in $\mathrm{AdS}_{4 / 7} \times \mathrm{S}^{7 / 4}$. It is shown that by fixing the $\kappa$-gauge symmetry and the reparametrization the non-relativistic action for AdS M2- and AdS M5-branes is reduced to a simple action which contains an additional term originating from the flux contribution of the WZ term. The non-relativistic flat M2-brane action given in [11] is reproduced as a flat limit of the non-relativistic AdS M2-brane action.

This paper is divided into the two parts. Sections 2-5 are devoted to studies of AdS branes in ten-dimensions, and those in eleven-dimensions are examined in sections 6-9. In section 2, $\mathrm{NH}$ superalgebras for branes in $\mathrm{AdS}_{5} \times \mathrm{S}^{5}$ are derived as IW contractions of the super- $\mathrm{AdS}_{5} \times \mathrm{S}^{5}$ algebra. It is shown that the directions along which the AdS brane worldvolume extends are restricted by requiring that the isometry on the AdS brane worldvolume and the Lorentz symmetry in the transverse space naturally extend to the super-isometry. The similar analyses are applied to branes in IIB pp-wave in section 3. WZ terms of AdS branes are derived by using the CE cohomology on the $\mathrm{AdS} \times \mathrm{S}$ superalgebra in section 4. Examining a non-relativistic limit of AdS brane actions, we obtain non-relativistic AdS brane actions in section 5. From section 6, M-theory in $\mathrm{AdS}_{4 / 7} \times \mathrm{S}^{7 / 4}$ is examined. We derive $\mathrm{NH}$ superalgebras for M-branes as IW contractions of the super- $\mathrm{AdS}_{4 / 7} \times \mathrm{S}^{7 / 4}$ algebras in section 6 . The similar analyses are applied to branes in $\mathrm{M}$ pp-wave in section 7. After deriving WZ terms of AdS M-branes by using the CE cohomology on the $\mathrm{AdS}_{4 / 7} \times \mathrm{S}^{7 / 4}$ superalgebras in section 8, we examine the non-relativistic limit of AdS M-brane actions in section 9. The last section is devoted to a summary and discussions.

The supervielbeins and the super spin-connections are given in Appendix A. In Appendix $[$, the $\kappa$-symmetry of Euclidean/Lorentzian brane actions is derived. Our construction of brane actions is applicable to branes in a pp-wave by taking the Penrose 
limit instead of non-relativistic limit. In fact, we derive brane actions in the pp-wave in Appendix C.

\section{NH Superalgebra of Branes in $\mathbf{A d S}_{5} \times \mathbf{S}^{5}$}

The super- $\mathrm{AdS}_{5} \times \mathrm{S}^{5}$ algebra, $\operatorname{psu}(2,2 \mid 4)$, is generated by translation $P_{A}=\left(P_{a}, P_{a^{\prime}}\right)$, Lorentz rotation $J_{A B}=\left(J_{a b}, J_{a^{\prime} b^{\prime}}\right)$ and Majorana-Weyl supercharges $Q_{I}(I=1,2)$ as

$$
\begin{aligned}
& {\left[P_{a}, P_{b}\right]=\lambda^{2} J_{a b}, \quad\left[P_{a^{\prime}}, P_{b^{\prime}}\right]=-\lambda^{2} J_{a^{\prime} b^{\prime}}} \\
& {\left[P_{a}, J_{b c}\right]=\eta_{a b} P_{c}-\eta_{a c} P_{b}, \quad\left[P_{a^{\prime}}, J_{b^{\prime} c^{\prime}}\right]=\eta_{a^{\prime} b^{\prime}} P_{c^{\prime}}-\eta_{a^{\prime} c^{\prime}} P_{b^{\prime}},} \\
& {\left[J_{a b}, J_{c d}\right]=\eta_{b c} J_{a d}+3 \text {-terms }, \quad\left[J_{a^{\prime} b^{\prime}}, J_{c^{\prime} d^{\prime}}\right]=\eta_{b^{\prime} c^{\prime}} J_{a^{\prime} d^{\prime}}+3 \text {-terms }} \\
& {\left[Q_{I}, P_{A}\right]=-\frac{\lambda}{2} Q_{J}\left(i \sigma_{2}\right)_{J I} \widehat{\Gamma}_{A}, \quad\left[Q_{I}, J_{A B}\right]=-\frac{1}{2} Q_{I} \Gamma_{A B},} \\
& \left\{Q_{I}, Q_{J}\right\}=2 i \mathcal{C} \Gamma^{A} \delta_{I J} h_{+} P_{A}-i \lambda \mathcal{C} \widehat{\Gamma}^{A B}\left(i \sigma_{2}\right)_{I J} h_{+} J_{A B}
\end{aligned}
$$

where $a=0, \cdots, 4$ and $a^{\prime}=5, \cdots, 9$ are vector indices of $\mathrm{AdS}_{5}$ and $\mathrm{S}^{5}$ respectively. The gamma matrix $\Gamma^{A} \in \operatorname{Spin}(1,9)$ satisfies

$$
\left\{\Gamma^{A}, \Gamma^{B}\right\}=2 \eta^{A B}, \quad\left(\Gamma^{A}\right)^{T}=-\mathcal{C} \Gamma^{A} \mathcal{C}^{-1}, \quad \mathcal{C}^{T}=-\mathcal{C}
$$

where $\mathcal{C}$ is the charge conjugation matrix. We use almost positive Minkowski metric $\eta_{A B}$ and define

$$
\begin{aligned}
& \widehat{\Gamma}_{A}=\left(-\Gamma_{a} \mathcal{I}, \Gamma_{a^{\prime}} \mathcal{J}\right), \quad \widehat{\Gamma}_{A B}=\left(-\Gamma_{a b} \mathcal{I}, \Gamma_{a^{\prime} b^{\prime}} \mathcal{J}\right), \quad \mathcal{I}=\Gamma^{01234}, \quad \mathcal{J}=\Gamma^{56789}, \\
& Q_{I} h_{+}=Q_{I}, \quad h_{+}=\frac{1}{2}\left(1+\Gamma_{11}\right), \quad \Gamma_{11}=\Gamma_{01 \cdots 9}
\end{aligned}
$$

and $\lambda=1 / R$ where $R$ is the radii of $\mathrm{AdS}_{5}$ and $\mathrm{S}^{5}$.

By using an element $g \in \operatorname{PSU}(2,2 \mid 4)$, a left-invariant (LI) Cartan one-form is defined as

$$
\Omega=g^{-1} d g \equiv \mathbf{L}^{A} P_{A}+\frac{1}{2} \mathbf{L}^{A B} J_{A B}+Q_{I} L^{I}
$$

Then the Maurer-Cartan (MC) equation, which is satisfied by LI Cartan one-forms

$$
d L^{\hat{A}}=\frac{1}{2} L^{\hat{B}} L^{\hat{C}} f_{\hat{C} \hat{B}}^{\hat{A}}, \quad \Omega=L^{\hat{A}} T_{\hat{A}}
$$


is equivalent to the superalgebra $\left[T_{\hat{A}}, T_{\hat{B}}\right\}=f_{\hat{A} \hat{B}} \hat{C} T_{\hat{C}}$. The Jacobi identities $f_{[\hat{A} \hat{B}} f_{|\hat{D}| \hat{C})}{ }^{\hat{E}}=$ 0 of the commutation relation of the superalgebra is stated as the nilpotency of the differential, $d^{2}=0$. Thus (2.1) is equivalent to

$$
\begin{aligned}
d \mathbf{L}^{A} & =-\eta_{B C} \mathbf{L}^{A B} \mathbf{L}^{C}+i \bar{L} \Gamma^{A} L \\
d \mathbf{L}^{a b} & =-\lambda^{2} \mathbf{L}^{a} \mathbf{L}^{b}-\eta_{c d} \mathbf{L}^{c a} \mathbf{L}^{b d}+i \lambda \bar{L} \Gamma^{a b} \mathcal{I} i \sigma_{2} L, \\
d \mathbf{L}^{a^{\prime} b^{\prime}} & =+\lambda^{2} \mathbf{L}^{a^{\prime}} \mathbf{L}^{b^{\prime}}-\eta_{c^{\prime} d^{\prime}} \mathbf{L}^{c^{\prime} a^{\prime}} \mathbf{L}^{b^{\prime} d^{\prime}}-i \lambda \bar{L} \Gamma^{a^{\prime} b^{\prime}} \mathcal{J} i \sigma_{2} L, \\
d L^{\alpha} & =-\frac{\lambda}{2} \mathbf{L}^{A} \widehat{\Gamma}_{A} i \sigma_{2} L-\frac{1}{4} \mathbf{L}^{A B} \Gamma_{A B} L .
\end{aligned}
$$

We derive NH superalgebras for AdS branes as IW contractions of the super- $\mathrm{AdS}_{5} \times \mathrm{S}^{5}$ algebra.

First we consider the bosonic subalgebra. Let us introduce the following coordinates:

$$
\bar{A}=A_{0}, \cdots, A_{p}, \quad \underline{A}=A_{p+1}, \cdots, A_{9},
$$

where $\bar{A}=\left(\bar{a}, \bar{a}^{\prime}\right)$ represent the worldvolume directions of the AdS brane. When the worldvolume extends along $m$ directions in $\mathrm{AdS}_{5}$ and $n$ directions in $\mathrm{S}^{5}$, we call it an $(m, n)$-brane. We rescale the generators as follow:

$$
P_{\underline{A}} \rightarrow \frac{1}{\Omega} P_{\underline{A}}, \quad J_{\bar{A} \underline{B}} \rightarrow \frac{1}{\Omega} J_{\bar{A} \underline{B}} .
$$

The limit $\Omega \rightarrow 0$ leads to the NH algebra for the AdS brane

$$
\begin{aligned}
& {\left[P_{\bar{a}}, P_{\bar{b}}\right]=\lambda^{2} J_{\bar{a} \bar{b}}, \quad\left[P_{\bar{a}^{\prime}}, P_{\bar{b}^{\prime}}\right]=-\lambda^{2} J_{\bar{a}^{\prime} \bar{b}^{\prime}},} \\
& {\left[P_{\bar{a}}, P_{\underline{b}}\right]=\lambda^{2} J_{\bar{a} \underline{b}}, \quad\left[P_{\bar{a}^{\prime}}, P_{\underline{b}^{\prime}}\right]=-\lambda^{2} J_{\bar{a}^{\prime} \underline{b^{\prime}}},} \\
& {\left[P_{\bar{A}}, J_{\bar{B} \bar{C}}\right]=\eta_{\bar{A} \bar{B}} P_{\bar{C}}-\eta_{\bar{A} \bar{C}} P_{\bar{B}}, \quad\left[P_{\underline{A}}, J_{\underline{B C}}\right]=\eta_{\underline{A B}} P_{\underline{C}}-\eta_{\underline{A C}} P_{\underline{B}},} \\
& {\left[P_{\bar{A}}, J_{\bar{B} \underline{C}}\right]=\eta_{\bar{A} \bar{B}} P_{\underline{C}},} \\
& {\left[J_{\bar{A} \bar{B}}, J_{\bar{C} \bar{D}}\right]=\eta_{\bar{A} \bar{D}} J_{\bar{B} \bar{C}}+3 \text {-terms }, \quad\left[J_{\underline{A B}}, J_{\underline{C D}}\right]=\eta_{\underline{A D}} J_{\underline{B C}}+3 \text {-terms },} \\
& {\left[J_{\bar{A} \bar{B}}, J_{\bar{C} \underline{D}}\right]=\eta_{\bar{B} \bar{C}} J_{\bar{A} \underline{D}}-\eta_{\bar{A} \bar{C}} J_{\bar{B} \underline{D}}, \quad\left[J_{\underline{A B}}, J_{\bar{C} \underline{D}}\right]=\eta_{\underline{B D}} J_{\bar{C} \underline{A}}-\eta_{\underline{A D}} J_{\bar{C} \underline{B}} .}
\end{aligned}
$$

This is the NH algebra of a brane given in [14] (see also [28]). The NH algebra contains two subalgebras. One is the isometry of $(m, n)$-brane worldvolume generated by $\left\{P_{\bar{A}}, J_{\bar{A} \bar{B}}\right\}$, the $\mathrm{AdS}_{m} \times \mathrm{S}^{n}$ algebra $\mathrm{so}(m-1,2) \times \mathrm{so}(n+1)$ for a Lorentzian brane and the $H^{m} \times \mathrm{S}^{n}$ algebra so $(m, 1) \times \operatorname{so}(n+1)$ for an Euclidean brane. The other is the Poincaré algebra, iso $(5-m) \times \operatorname{iso}(5-n)$ for a Lorentzian brane and iso $(4-m, 1) \times \operatorname{iso}(5-n)$ for a Euclidean 
brane, generated by $\left\{P_{\underline{A}}, J_{\underline{A B}}\right\}$ which is the isometry of the transverse space $\mathbb{E}^{5-m} \times \mathbb{E}^{5-n}$ and $\mathbb{E}^{4-m, 1} \times \mathbb{E}^{5-n}$ respectively.

Next, we consider the fermionic part. Let us introduce a condition

$$
\theta=M \theta \quad \text { with } \quad M=\ell \Gamma^{\bar{A}_{0} \cdots \bar{A}_{p}} \otimes \rho
$$

where $\ell^{2}(-1)^{\left[\frac{p+1}{2}\right]} \rho^{2}=1$ for $M^{2}=1$. The $2 \times 2$ matrix $\rho$ is determined below. As $\theta=h_{+} \theta,\left[M, h_{+}\right]=0$ is required so that $p=\operatorname{odd}$. We demand that $M$ satisfies following relations

$$
\begin{aligned}
M^{\prime} \Gamma^{\bar{A}} & =\Gamma^{\bar{A}} M, \\
M^{\prime} \widehat{\Gamma}^{\bar{A} \bar{B}} i \sigma_{2} & =\widehat{\Gamma}^{\bar{A} \bar{B}} i \sigma_{2} M,
\end{aligned}
$$

where $M^{\prime}=C^{-1} M^{T} C$. If these are satisfied, the isometry of the AdS brane worldvolume and the Lorentz symmetry in the transverse space, $\operatorname{so}(m-1,2) \times \operatorname{so}(n+1) \times \operatorname{so}(5-$ $m) \times \operatorname{so}(5-n)$ for a Lorentzian brane and $\operatorname{so}(m, 1) \times \operatorname{so}(n+1) \times \operatorname{so}(4-m, 1) \times \operatorname{so}(5-n)$ for a Euclidean brane, naturally extend to the super-isometry as will be seen below. It is straightforward to see that the first condition is satisfied by $\rho^{T}=\rho$ for $p=1 \bmod 4$ and by $\rho^{T}=-\rho$ for $p=3 \bmod 4$. The second condition restricts the direction along which branes extend. Since, for $\rho=1(p=1 \bmod 4)$ and $\rho=i \sigma_{2}(p=3 \bmod 4)$, we derive

$$
M^{\prime} \widehat{\Gamma}^{\bar{A} \bar{B}} i \sigma_{2}=(-1)^{\mathrm{d}} \widehat{\Gamma}^{\bar{A} \bar{B}} i \sigma_{2} M
$$

we have (odd,odd)-branes. d denotes the number of Dirichlet directions contained in $\mathrm{AdS}_{5}$. On the other hand, for $\rho=\sigma_{1}, \sigma_{3}(p=1 \bmod 4)$, since

$$
M^{\prime} \widehat{\Gamma}^{\bar{A} \bar{B}} i \sigma_{2}=-(-1)^{\mathrm{d}} \widehat{\Gamma}^{\bar{A} \bar{B}} i \sigma_{2} M
$$

(even,even)-branes are allowed. In both cases, we have $\ell=\sqrt{-s}$ and

$$
M^{\prime}=-M
$$

We summarize branes in Table 1. The 9-brane is nothing but $\mathrm{AdS}_{5} \times \mathrm{S}^{5}$ itself as $M=h_{+}$ in this case. The (even,even)-branes $(p=1 \bmod 4)$ and (odd,odd)-branes $(p=3 \bmod 4)$ are 1/2 BPS Dirichlet branes of $\mathrm{F}$ - and D-strings in $\mathrm{AdS}_{5} \times \mathrm{S}^{5}$ derived in [16-18 $]^{1}$. In the presence of gauge field condensates, see [19]. As will be seen in section 4, we find consistent non-relativistic limits for these AdS branes.

\footnotetext{
${ }^{1}$ The brane probe analysis [29] is also consistent with this result.
} 


\begin{tabular}{|c|c|c|c|c|c|}
\hline$\rho$ & 1-brane & 3-brane & 5-brane & 7-brane & 9-brane \\
\hline$\sigma_{1}, \sigma_{3}$ & $(2,0),(0,2)$ & & $(4,2),(2,4)$ & & \\
$i \sigma_{2}$ & & $(3,1),(1,3)$ & & $(5,3),(3,5)$ & \\
1 & $(1,1)$ & & $(5,1),(3,3),(1,5)$ & & $(5,5)$ \\
\hline
\end{tabular}

Table 1: Branes in $\mathrm{AdS}_{5} \times \mathrm{S}^{5}$

Let us decompose $Q^{\alpha}$ with the projection operator

$$
\mathcal{P}_{ \pm}=\frac{1}{2}(1 \pm M) \quad \text { as } \quad Q=Q_{+}+Q_{-}, \quad Q_{ \pm} \mathcal{P}_{ \pm}=Q_{ \pm},
$$

and rescale fermionic generators as

$$
Q_{+} \rightarrow Q_{+}, \quad Q_{-} \rightarrow \frac{1}{\Omega} Q_{-} .
$$

Taking $\Omega \rightarrow 0$ leads to (anti-)commutation relations

$$
\begin{aligned}
& {\left[P_{\bar{A}}, Q_{+}\right]=\frac{\lambda}{2} Q_{+} \widehat{\Gamma}_{\bar{A}} i \sigma_{2}, \quad\left[P_{\underline{A}}, Q_{+}\right]=\frac{\lambda}{2} Q_{-} \widehat{\Gamma}_{\underline{A}} i \sigma_{2}, \quad\left[P_{\bar{A}}, Q_{-}\right]=\frac{\lambda}{2} Q_{-} \widehat{\Gamma}_{\bar{A}} i \sigma_{2},} \\
& {\left[J_{\bar{A} \bar{B}}, Q_{ \pm}\right]=\frac{1}{2} Q_{ \pm} \Gamma_{\bar{A} \bar{B}}, \quad\left[J_{\underline{A B}}, Q_{ \pm}\right]=\frac{1}{2} Q_{ \pm} \Gamma_{\underline{A B}}, \quad\left[J_{\bar{A} \underline{B}}, Q_{+}\right]=\frac{1}{2} Q_{-} \Gamma_{\bar{A} \underline{B}}} \\
& \left\{Q_{+}, Q_{+}\right\}=2 i \mathcal{C} \Gamma^{\bar{A}} h_{+} \mathcal{P}_{+} P_{\bar{A}}-i \lambda \mathcal{C} \widehat{\Gamma}^{\bar{A} \bar{B}} i \sigma_{2} h_{+} \mathcal{P}_{+} J_{\bar{A} \bar{B}}-i \lambda \mathcal{C} \widehat{\Gamma}^{A B} i \sigma_{2} h_{+} \mathcal{P}_{+} J_{\underline{A B}} \\
& \left\{Q_{+}, Q_{-}\right\}=2 i \mathcal{C} \Gamma^{\underline{A}} h_{+} \mathcal{P}_{-} P_{\underline{A}}-2 i \lambda \mathcal{C} \widehat{\Gamma}^{\bar{A}} \underline{B} i \sigma_{2} h_{+} \mathcal{P}_{-} J_{\bar{A} \underline{B}} .
\end{aligned}
$$

In summary, we have derived the NH superalgebra for AdS brane, (2.9) and (2.18), as an IW contraction of $\mathrm{psu}(2,2 \mid 4)$. The NH superalgebra for an F-string [15] is contained as the $p=1$ case.

We note that generators $P_{\bar{A}}, J_{\bar{A} \bar{B}}, J_{\underline{A B}}$ and $Q_{+}$form a super-subalgebra

$$
\begin{aligned}
& {\left[P_{\bar{a}}, P_{\bar{b}}\right]=\lambda^{2} J_{\bar{a} \bar{b}}, \quad\left[P_{\bar{a}^{\prime}}, P_{\bar{b}^{\prime}}\right]=-\lambda^{2} J_{\bar{a}^{\prime} \bar{b}^{\prime}}, \quad\left[P_{\bar{A}}, J_{\bar{B} \bar{C}}\right]=\eta_{\bar{A} \bar{B}} P_{\bar{C}}-\eta_{\bar{A} \bar{C}} P_{\bar{B}},} \\
& {\left[J_{\bar{A} \bar{B}}, J_{\bar{C} \bar{D} \bar{D}}\right]=\eta_{\bar{A} \bar{D}} J_{\bar{B} \bar{C}}+3 \text {-terms }, \quad\left[J_{\underline{A B}}, J_{\underline{C D}}\right]=\eta_{\underline{A D}} J_{\underline{B C}}+3 \text {-terms },} \\
& {\left[P_{\bar{A}}, Q_{+}\right]=\frac{\lambda}{2} Q_{+} \widehat{\Gamma}_{\bar{A}} i \sigma_{2}, \quad\left[J_{\bar{A} \bar{B}}, Q_{+}\right]=\frac{1}{2} Q_{+} \Gamma_{\bar{A} \bar{B}}, \quad\left[J_{\underline{A B}}, Q_{+}\right]=\frac{1}{2} Q_{+} \Gamma_{\underline{A B}},} \\
& \left\{Q_{+}, Q_{+}\right\}=2 i \mathcal{C} \Gamma^{\bar{A}} h_{+} \mathcal{P}_{+} P_{\bar{A}}-i \lambda \mathcal{C} \widehat{\Gamma}^{\bar{A} \bar{B}} i \sigma_{2} h_{+} \mathcal{P}_{+} J_{\bar{A} \bar{B}}-i \lambda \mathcal{C} \widehat{\Gamma}^{\underline{A B}} i \sigma_{2} h_{+} \mathcal{P}_{+} J_{\underline{A B}},
\end{aligned}
$$

which is a supersymmetrization of $\operatorname{so}(m-1,2) \times \operatorname{so}(n+1) \times \operatorname{so}(5-m) \times \operatorname{so}(5-n)$ for a Lorentzian brane and $\mathrm{so}(m, 1) \times \operatorname{so}(n+1) \times \operatorname{so}(4-m, 1) \times \operatorname{so}(5-n)$ for a Euclidean brane. The superalgebra for the $(5,5)$-brane is $\mathrm{psu}(2,2 \mid 4)$. Since the dimension of the bosonic subalgebra is 14 for $(1,1)$-, $(3,1)$-, $(1,3)$ - and (3,3)-branes, 16 for $(2,0)$-, $(0,2)$-, $(4,2)$-, 
(2,4)-branes, and 22 for (5,1)-, (1,5)-, (5,3)- and (3,5)-branes, one may guess the corresponding superalgebras as those including variants of $\operatorname{su}(2 \mid 2) \times \operatorname{su}(2 \mid 2), \operatorname{osp}(4 \mid 4)$ and $\operatorname{osp}(6 \mid 2) \times \operatorname{psu}(2 \mid 1)$, respectively. The existence of these superalgebras is ensured by (2.11) and (2.12).

It is straightforward to derive $\mathrm{MC}$ equations for the AdS brane $\mathrm{NH}$ superalgebra (2.9) and (2.18)

$$
\begin{aligned}
& d \mathbf{L}^{\bar{A}}=-\eta_{\bar{B} \bar{C}} \mathbf{L}^{\bar{A} \bar{B}} \mathbf{L}^{\bar{C}}+i \bar{L}_{+} \Gamma^{\bar{A}} L_{+}, \\
& d \mathbf{L}^{\underline{A}}=-\eta_{\bar{B} \bar{C}} \mathbf{L}^{\underline{A}} \bar{B} \mathbf{L}^{\bar{C}}-\eta_{\underline{B C}} \mathbf{L}^{\underline{A B}} \mathbf{L}^{\underline{C}}+i \bar{L}_{+} \Gamma^{\underline{A}} L_{-}+i \bar{L}_{-} \Gamma^{\underline{A}} L_{+}, \\
& d \mathbf{L}^{\bar{a} \bar{b}}=-\lambda^{2} \mathbf{L}^{\bar{a}} \mathbf{L}^{\bar{b}}-\eta_{\bar{c} \bar{d}} \mathbf{L}^{\bar{c} \bar{a}} \mathbf{L}^{\bar{b} \bar{d}}-i \lambda \bar{L}_{+} \widehat{\Gamma}^{\bar{a} \bar{b}} i \sigma_{2} L_{+}, \\
& d \mathbf{L}^{\bar{a}^{\prime} \bar{b}^{\prime}}=+\lambda^{2} \mathbf{L}^{\bar{a}^{\prime}} \mathbf{L}^{\bar{b}^{\prime}}-\eta_{\bar{c}^{\prime}} \bar{d}^{\prime} \mathbf{L}^{\bar{c}^{\prime} \bar{a}^{\prime}} \mathbf{L}^{\bar{b}^{\prime} \bar{d}^{\prime}}-i \lambda \bar{L}_{+} \widehat{\Gamma}^{\bar{a}^{\prime} \bar{b}^{\prime}} i \sigma_{2} L_{+}, \\
& d \mathbf{L} \underline{A B}=-\eta_{\underline{C D}} \mathbf{L} \frac{C A}{L} \mathbf{L} \underline{B D}-i \lambda \bar{L}_{+} \widehat{\Gamma} \underline{A B} i \sigma_{2} L_{+}, \\
& d \mathbf{L}^{\bar{a} \underline{b}}=-\lambda^{2} \mathbf{L}^{\bar{a}} \mathbf{L}^{\underline{b}}-\eta_{\bar{c} \bar{d}} \mathbf{L}^{\bar{c} \bar{a}} \mathbf{L}^{\underline{b} \bar{d}}-\eta_{\underline{c} \underline{ }} \mathbf{L}^{\underline{c} \bar{a}} \mathbf{L}^{\underline{b d}} \\
& -i \lambda \bar{L}_{+} \widehat{\Gamma}^{\bar{a}} \underline{b} i \sigma_{2} L_{-}-i \lambda \bar{L}_{-} \widehat{\Gamma}^{\bar{a}} \underline{b} i \sigma_{2} L_{+}, \\
& d \mathbf{L}^{\bar{a}^{\prime} \underline{\underline{b}}^{\prime}}=+\lambda^{2} \mathbf{L}^{\bar{a}^{\prime}} \mathbf{L}^{\underline{b^{\prime}}}-\eta_{\bar{c}^{\prime} \overline{d^{\prime}}} \mathbf{L}^{\bar{c}^{\prime} \bar{a}^{\prime}} \mathbf{L}^{\underline{b}^{\prime} \bar{d}^{\prime}}-\eta_{\underline{c^{\prime}} \underline{d}^{\prime}} \mathbf{L}^{\underline{c}^{\prime} \bar{a}^{\prime}} \mathbf{L}^{\underline{b}^{\prime} \underline{d}^{\prime}} \\
& -i \lambda \bar{L}_{+} \widehat{\Gamma}^{\bar{a}^{\prime} \underline{b}^{\prime}} i \sigma_{2} L_{-}-i \lambda \bar{L}_{-} \widehat{\Gamma}^{\bar{a}^{\prime} \underline{b}^{\prime}} i \sigma_{2} L_{+}, \\
& d L_{+}=-\frac{\lambda}{2} \mathbf{L}^{\bar{A}} \widehat{\Gamma}_{\bar{A}} i \sigma_{2} L_{+}-\frac{1}{4} \mathbf{L}^{\bar{A} \bar{B}} \Gamma_{\bar{A} \bar{B}} L_{+}-\frac{1}{4} \mathbf{L} \underline{A B} \Gamma_{\underline{A B}} L_{+}, \\
& d L_{-}=-\frac{\lambda}{2} \mathbf{L}^{\bar{A}} \widehat{\Gamma}_{\bar{A}} i \sigma_{2} L_{-}-\frac{\lambda}{2} \mathbf{L} \underline{\underline{A}} \widehat{\Gamma}_{\underline{A}} i \sigma_{2} L_{+} \\
& -\frac{1}{4} \mathbf{L}^{\bar{A} \bar{B}} \Gamma_{\bar{A} \bar{B}} L_{-}-\frac{1}{4} \mathbf{L}^{\underline{A B}} \Gamma_{\underline{A B}} L_{-}-\frac{1}{2} \mathbf{L}^{\bar{A}} \underline{\underline{B}} \Gamma_{\bar{A} \underline{B}} L_{+} .
\end{aligned}
$$

An alternative way to derive these $\mathrm{MC}$ equations is to rescale the Cartan one-forms in the MC equation (2.6) as

$$
\mathbf{L}^{\underline{A}} \rightarrow \Omega \mathbf{L}^{\underline{A}}, \quad \mathbf{L}^{\bar{A} \underline{B}} \rightarrow \Omega \mathbf{L}^{\bar{A} \underline{B}}, \quad L_{-} \rightarrow \Omega L_{-}
$$

and take the limit $\Omega \rightarrow 0$. This provides the leading order terms of the expansion considered in the non-relativistic limit in section 4.

Finally, let us consider an alternative scaling

$$
\lambda \rightarrow \frac{1}{\omega} \lambda, \quad P_{\bar{A}} \rightarrow \frac{1}{\omega} P_{\bar{A}}, \quad J_{\bar{A} \underline{B}} \rightarrow \omega J_{\bar{A} \underline{B}}, \quad Q_{+} \rightarrow \frac{1}{\sqrt{\omega}} Q_{+}, \quad Q_{-} \rightarrow \sqrt{\omega} Q_{-} .
$$

Since $\lambda$ is absorbed as

$$
P_{\bar{A}} \rightarrow \frac{1}{\lambda} P_{\bar{A}}, \quad P_{\underline{A}} \rightarrow \frac{1}{\lambda} P_{\underline{A}}, \quad Q_{ \pm} \rightarrow \frac{1}{\sqrt{\lambda}} Q_{ \pm},
$$

this is equivalent to (2.8) and (2.17) with $\Omega=1 / \omega$. In this paper, we use (2.8) and (2.17) instead of (2.30), though both limits lead to the same results. 


\section{NH Superalgebra of Branes in IIB PP-Wave}

Type IIB PP-wave superalgebra is obtained as an IW contraction of the super- $\mathrm{AdS}_{5} \times \mathrm{S}^{5}$ algebra. First of all, let us introduce the following quantities for later convenience,

$$
\begin{aligned}
& P_{ \pm}=\frac{1}{\sqrt{2}}\left(P_{9} \pm P_{0}\right), \quad P_{\hat{i}}^{*}=\left(P_{i}^{*}=J_{0 i}, P_{i^{\prime}}^{*}=J_{9 i^{\prime}}\right) \\
& Q=Q^{(+)}+Q^{(-)}, \quad Q^{( \pm)}=Q^{( \pm)} \ell_{ \pm}, \quad \ell_{ \pm}=\frac{1}{2} \Gamma_{ \pm} \Gamma_{\mp}, \quad \Gamma_{ \pm}=\frac{1}{\sqrt{2}}\left(\Gamma_{9} \pm \Gamma_{0}\right)
\end{aligned}
$$

where $i=1,2,3,4$ and $i^{\prime}=5,6,7,8$. The IW contraction is performed in [5] by scaling generators in the super- $\mathrm{AdS}_{5} \times \mathrm{S}^{5}$ algebra as

$$
P_{+} \rightarrow \frac{1}{\Lambda^{2}} P_{+}, \quad P_{\hat{i}} \rightarrow \frac{1}{\Lambda} P_{\hat{i}}, \quad P_{\hat{i}}^{*} \rightarrow \frac{1}{\Lambda} P_{\hat{i}}^{*}, \quad Q^{(+)} \rightarrow \frac{1}{\Lambda} Q^{(+)},
$$

and then taking the limit $\Lambda \rightarrow 0$. After the contraction, the super- $\operatorname{AdS}_{5} \times \mathrm{S}^{5}$ algebra is reduced to the IIB pp-wave superalgebra

$$
\begin{aligned}
& {\left[P_{-}, P_{\hat{i}}\right]=-\frac{\lambda^{2}}{\sqrt{2}} P_{\hat{i}}^{*}, \quad\left[P_{-}, P_{\hat{i}}^{*}\right]=\frac{1}{\sqrt{2}} P_{\hat{i}}, \quad\left[P_{\hat{i}}, P_{\hat{j}}^{*}\right]=-\frac{1}{\sqrt{2}} \eta_{\hat{i} \hat{j}} P_{+}} \\
& {\left[P_{\hat{i}}, J_{\hat{j} \hat{k}}\right]=\eta_{\hat{i} \hat{j}} P_{\hat{k}}-\eta_{\hat{i} \hat{k}} P_{\hat{j}}, \quad\left[P_{\hat{i}}^{*}, J_{\hat{j} \hat{k}}\right]=\eta_{\hat{i} \hat{j}} P_{\hat{k}}^{*}-\eta_{\hat{i} \hat{k}} P_{\hat{j}}^{*}, \quad\left[J_{\hat{i} \hat{j}}, J_{\hat{k} \hat{l}}\right]=\eta_{\hat{j} \hat{k}} J_{\hat{i} \hat{l}}+3 \text {-terms }} \\
& {\left[Q^{(-)}, P_{\hat{i}}\right]=\frac{1}{2} Q^{(+)} \Gamma_{\hat{i}} \mathcal{I}_{i} \sigma_{2}, \quad\left[Q^{(+)}, P_{-}\right]=-\frac{1}{2} Q^{(+)} \Gamma_{+} \mathcal{I}_{i} \sigma_{2}} \\
& {\left[Q^{(-)}, P_{\hat{i}}^{*}\right]=\frac{1}{2 \sqrt{2}} Q^{(+)} \Gamma_{+} \Gamma_{\hat{i}}, \quad\left[Q^{( \pm)}, J_{\hat{i} \hat{j}}\right]=-\frac{1}{2} Q^{( \pm)} \Gamma_{\hat{i} \hat{j}}} \\
& \left\{Q^{(+)}, Q^{(+)}\right\}=2 i \mathcal{C} \Gamma_{-} P_{+}, \\
& \left\{Q^{(-)}, Q^{(-)}\right\}=2 i \mathcal{C} \Gamma_{+} P_{-}-i \frac{\lambda}{\sqrt{2}} \mathcal{C} \widehat{\Gamma}^{\hat{i} \hat{j}} i \sigma_{2} J_{\hat{i} \hat{j}} \\
& \left\{Q^{( \pm)}, Q^{(\mp)}\right\}=2 i \mathcal{C} \Gamma^{\hat{i}} \ell_{\mp} P_{\hat{i}}+i \lambda \mathcal{C} \widehat{\Gamma}^{\hat{i}} \ell_{\mp} i \sigma_{2} P_{\hat{i}}^{*}
\end{aligned}
$$

where $\widehat{\Gamma}^{\hat{i} j}=\left(-\Gamma^{i j} \Gamma_{+} f, \Gamma^{i^{\prime} j^{\prime}} \Gamma_{+} g\right), \widehat{\Gamma}^{\hat{i}}=\left(\Gamma^{i} f, \Gamma^{i^{\prime}} g\right), f=\Gamma^{1234}$ and $g=\Gamma^{5678}$. The bosonic subalgebra, the pp-wave algebra, is the semi-direct product of the Heisenberg algebra generated by $\left\{P_{\hat{i}}, P_{\hat{i}}^{*}\right\}$ with an outer automorphism $P_{-}$and the Lorentz algebra generated by $J_{\hat{i} \hat{j}}$.

\subsection{Lorentzian branes}

Here we consider the case that $(+,-)$ are contained in the Neumann directions. Let us denote the Neumann and the Dirichlet directions, respectively, as

$$
\bar{A}=(+,-, \overline{\hat{i}}), \quad \underline{A}=\underline{\hat{i}} .
$$


We derive the NH superalgebra of a Lorentzian pp-wave brane as an IW contraction of the pp-wave superalgebra.

Let us first consider the bosonic subalgebra. We rescale generators in the pp-wave algebra as

$$
P_{\underline{A}} \rightarrow \frac{1}{\Omega} P_{\underline{A}}, \quad J_{\underline{\hat{i}} \hat{j}} \rightarrow \frac{1}{\Omega} J_{\underline{\hat{i}} \underline{\hat{j}}}, \quad P_{\underline{\hat{i}}}^{*} \rightarrow \frac{1}{\Omega} P_{\underline{\hat{i}}}^{*},
$$

and then take the limit $\Omega \rightarrow 0$. The resulting algebra is the $\mathrm{NH}$ algebra of a pp-wave brane

$$
\begin{aligned}
& {\left[P_{-}, P_{\hat{i}}\right]=-\frac{\lambda^{2}}{\sqrt{2}} P_{\hat{\hat{i}}}^{*}, \quad\left[P_{-}, P_{\underline{\hat{i}}}\right]=-\frac{\lambda^{2}}{\sqrt{2}} P_{\underline{\hat{i}}}^{*}, \quad\left[P_{-}, P_{\hat{\hat{i}}}^{*}\right]=\frac{1}{\sqrt{2}} P_{\hat{\hat{i}}}, \quad\left[P_{-}, P_{\underline{\hat{i}}}^{*}\right]=\frac{1}{\sqrt{2}} P_{\underline{\hat{i}}},} \\
& {\left[P_{\overline{\hat{i}}}, P_{\hat{\hat{i}}}^{*}\right]=-\frac{1}{\sqrt{2}} \eta_{\overline{\hat{i}} \overline{\hat{j}}} P_{+}, \quad\left[P_{\hat{\hat{i}}}, J_{\overline{\hat{j}} \underline{\hat{k}}}\right]=\eta_{\overline{\hat{i}} \overline{\hat{j}}} P_{\underline{\hat{k}}}, \quad\left[J_{\overline{\hat{i}} \underline{\hat{j}}}, P_{\hat{\hat{k}}}^{*}\right]=-\eta_{\overline{\hat{i}} \overline{\hat{k}}} P_{\underline{\hat{j}}}^{*},}
\end{aligned}
$$

and

$$
\begin{aligned}
& {\left[P_{\hat{\hat{i}}}, J_{\overline{\hat{j}} \overline{\hat{k}}}\right]=\eta_{\overline{\hat{i} \hat{j}}} P_{\hat{\hat{k}}}-\eta_{\overline{\hat{i}} \overline{\hat{k}}} P_{\overline{\hat{j}}}, \quad\left[P_{\underline{\hat{i}}}, J_{\underline{\hat{j}} \underline{\hat{k}}}\right]=\eta_{\underline{\hat{i}} \underline{\hat{j}}} P_{\hat{\hat{k}}}-\eta_{\underline{\hat{i}} \underline{\hat{k}}} P_{\hat{\hat{j}}},}
\end{aligned}
$$

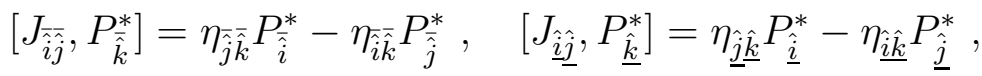

$$
\begin{aligned}
& {\left[J_{\overline{\hat{i} \bar{j}}}, J_{\overline{\hat{k}} \overline{\hat{l}}}\right]=\eta_{\overline{\bar{j}} \overline{\hat{k}}} J_{\overline{\hat{i}} \overline{\hat{l}}}+3 \text {-terms }, \quad\left[J_{\underline{\hat{i}} \underline{\hat{j}}}, J_{\underline{\hat{k} \hat{l}}}\right]=\eta_{\underline{\hat{j}} \underline{\hat{k}}} J_{\underline{\hat{i} \hat{l}}}+3 \text {-terms },}
\end{aligned}
$$

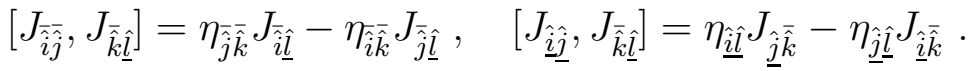

Next we consider the fermionic part. We introduce a matrix $M$

$$
M=\ell \Gamma^{+-\bar{A}_{1} \cdots \bar{A}_{p-1} \rho}
$$

where $\rho$ is a $2 \times 2$ matrix. Then $Q^{( \pm)}$are decomposed into the two parts as follows:

$$
Q_{ \pm}^{(\bullet)}= \pm Q_{ \pm}^{(\bullet)} M
$$

The chirality of $Q^{(\bullet)}$ is preserved only when $p=$ odd. In addition, requiring that $M^{2}=1$, we obtain the following condition,

$$
\ell^{2}(-1)^{\left[\frac{p-1}{2}\right]} \rho^{2}=1
$$

Then we demand that

$$
\begin{gathered}
M^{\prime} \Gamma^{\bar{A}}=\Gamma^{\bar{A}} M, \\
M^{\prime} \widehat{\Gamma}^{\overline{\hat{i}} \overline{\hat{j}}} i \sigma_{2}=\widehat{\Gamma}^{\bar{i} \overline{\hat{j}}} i \sigma_{2} M
\end{gathered}
$$


where

$$
M^{\prime}=\mathcal{C}^{-1} M^{T} \mathcal{C}= \pm(-1)^{p+\left[\frac{p-1}{2}\right]} M, \quad \rho^{T}= \pm \rho
$$

Since

$$
M^{\prime} \Gamma^{\bar{A}}= \pm(-1)^{\left[\frac{p-1}{2}\right]} \Gamma^{\bar{A}} M, \quad \rho^{T}= \pm \rho
$$

the first condition is satisfied by

$$
\pm(-1)^{\left[\frac{p-1}{2}\right]}=1, \quad \rho^{T}= \pm \rho
$$

This implies that $\rho^{T}=\rho$ for $p=1 \bmod 4$ and $\rho^{T}=-\rho$ for $p=3 \bmod 4$, and that $M^{\prime}=-M$ and $\ell=1$. The second condition is rewritten as

$$
\pm(-1)^{\mathrm{n}}=1, \quad \rho^{T}=\left\{\begin{array}{c}
1, i \sigma_{2} \\
\sigma_{1}, \sigma_{3}
\end{array}\right.
$$

where $\mathrm{n}$ is the number of the Neumann directions contained in $\{1,2,3,4\}$ and $\{5,6,7,8\}$ so that the directions along which a pp-wave brane worldvolume extends are restricted. We summarize the results in Table 2 (+,-;odd,odd)-branes with $p=1 \bmod 4$ and

\begin{tabular}{|c|c|c|c|c|c|}
\hline$\rho$ & 1-brane & 3-brane & 5-brane & 7-brane & 9-brane \\
\hline$\sigma_{1}, \sigma_{3}$ & & & $\begin{array}{c}(+,-; 1,3) \\
(+,-; 3,1)\end{array}$ & & \\
& & & & $(+,-; 4,2)$ & \\
\hline$\sigma_{2}$ & & $\begin{array}{r}(+,-; 0,2) \\
(+,-; 2,0)\end{array}$ & & $(+,-; 2,4)$ & \\
\hline 1 & $(+,-)$ & & $\begin{array}{c}(+,-; 0,4) \\
(+,-; 2,2) \\
(+,-; 4,0)\end{array}$ & & $(+,-; 4,4)$ \\
& & & & & \\
\hline
\end{tabular}

Table 2: Lorentzian pp-wave branes.

(+,-;even,even)-branes with $p=3 \bmod 4$ are $1 / 2$ BPS D-branes of an open pp-wave superstring $[16,30]$. Our results are consistent with those obtained in the brane probe analysis [29], the supergravity analysis [31] and the CFT analysis in the light-cone gauge [32-35]. 
Scaling $Q_{ \pm}^{(\bullet)}$ as

$$
Q_{+}^{(\bullet)} \rightarrow Q_{+}^{(\bullet)}, \quad Q_{-}^{(\bullet)} \rightarrow \frac{1}{\Omega} Q_{-}^{(\bullet)}
$$

and taking the limit $\Omega \rightarrow 0$, we obtain the fermionic part of the NH superalgebra

$$
\begin{aligned}
& {\left[Q_{ \pm}^{(-)}, P_{\hat{i}}\right]=-\frac{1}{2 \sqrt{2}} Q_{ \pm}^{(+)} \Gamma_{\hat{\hat{i}}} \Gamma_{+} f i \sigma_{2}, \quad\left[Q_{+}^{(-)}, P_{\underline{\hat{i}}}\right]=-\frac{1}{2 \sqrt{2}} Q_{-}^{(+)} \Gamma_{\underline{\hat{i}}} \Gamma_{+} f i \sigma_{2},} \\
& {\left[Q_{ \pm}^{(+)}, P_{-}\right]=-\frac{1}{\sqrt{2}} Q_{ \pm}^{(+)} f i \sigma_{2}, \quad\left[Q_{ \pm}^{(-)}, P_{\hat{i}}^{*}\right]=\frac{1}{2 \sqrt{2}} Q_{ \pm}^{(+)} \Gamma_{+} \Gamma_{\overline{\hat{i}}},} \\
& {\left[Q_{+}^{(-)}, P_{\underline{\hat{i}}}^{*}\right]=\frac{1}{2 \sqrt{2}} Q_{-}^{(+)} \Gamma_{+} \Gamma_{\underline{\hat{i}}}, \quad\left[Q_{ \pm}^{(\bullet)}, J_{\overline{\hat{i}} \overline{\hat{j}}}\right]=-\frac{1}{2} Q_{ \pm}^{(\bullet)} \Gamma_{\overline{\hat{i}} \overline{\hat{j}}}, \quad\left[Q_{ \pm}^{(\bullet)}, J_{\underline{\hat{i}} \underline{\hat{j}}}\right]=-\frac{1}{2} Q_{ \pm}^{(\bullet)} \Gamma_{\underline{\hat{i}} \underline{\hat{j}}},} \\
& {\left[Q_{+}^{( \pm)}, J_{\underline{\hat{i}} \underline{\hat{j}}}\right]=-\frac{1}{2} Q_{-}^{( \pm)} \Gamma_{\underline{\hat{i}} \underline{\hat{j}}}, \quad\left\{Q_{+}^{(+)}, Q_{+}^{(+)}\right\}=2 i \mathcal{C} \Gamma_{-} \mathcal{P}_{+} P_{+},} \\
& \left\{Q_{+}^{(-)}, Q_{+}^{(-)}\right\}=2 i \mathcal{C} \Gamma_{+} \mathcal{P}_{-} P_{+}-i \frac{\lambda}{\sqrt{2}} \mathcal{C} \widehat{\Gamma}^{\overline{\hat{i}} \overline{\hat{j}}} i \sigma_{2} \ell_{-} \mathcal{P}_{+} J_{\overline{\hat{i}} \overline{\hat{j}}}-i \frac{\lambda}{\sqrt{2}} \mathcal{C} \widehat{\Gamma}^{\hat{\hat{i}} \underline{\hat{j}}} i \sigma_{2} \ell_{-} \mathcal{P}_{+} J_{\underline{\underline{\hat{j}}} \hat{\hat{j}}}, \\
& \left\{Q_{ \pm}^{(-)}, Q_{\mp}^{(-)}\right\}=-2 i \frac{\lambda}{\sqrt{2}} \mathcal{C} \widehat{\Gamma}^{\overline{\hat{i}} \hat{\hat{j}}} i \sigma_{2} \ell_{-} \mathcal{P}_{\mp} J_{\widehat{\hat{i}} \hat{\hat{j}}}, \\
& \left\{Q_{+}^{(+)}, Q_{+}^{(-)}\right\}=2 i \mathcal{C} \Gamma^{\overline{\hat{i}}} \ell_{-} \mathcal{P}_{+} P_{\hat{i}}+i \lambda \mathcal{C} \widehat{\Gamma}^{\hat{i}} i \sigma_{2} \ell_{-} \mathcal{P}_{+} P_{\hat{i}}^{*}, \\
& \left\{Q_{ \pm}^{(+)}, Q_{\mp}^{(-)}\right\}=2 i \mathcal{C} \Gamma^{\underline{\hat{i}}} \ell_{-} \mathcal{P}_{\mp} P_{\underline{\hat{i}}}+i \lambda \mathcal{C} \widehat{\Gamma}^{\hat{\hat{i}}} i \sigma_{2} \ell_{-} \mathcal{P}_{\mp} P_{\underline{\hat{i}}}^{*} \text {. }
\end{aligned}
$$

In summary we have obtained the $\mathrm{NH}$ superalgebra of a pp-wave brane as (3.6), (3.7) and (3.18). This superalgebra can be derived from the NH superalgebra of an AdS brane (2.9) and (2.18) by an IW contraction.

We note that the NH superalgebra of a pp-wave brane contains a super-subalgebra generated by $P_{ \pm}, P_{\hat{i}}, P_{\hat{i}}^{*}, J_{\hat{\hat{i}} \overline{\hat{j}}}, J_{\hat{i} \hat{\hat{j}} \underline{ }}$ and $Q_{+}^{( \pm)}$

$$
\begin{aligned}
& {\left[P_{-}, P_{\hat{i}}\right]=-\frac{\lambda^{2}}{\sqrt{2}} P_{\hat{i}}^{*}, \quad\left[P_{-}, P_{\hat{i}}^{*}\right]=\frac{1}{\sqrt{2}} P_{\hat{i}}, \quad\left[P_{\hat{i}}, P_{\hat{i}}^{*}\right]=-\frac{1}{\sqrt{2}} \eta_{\bar{i} \overline{\hat{j}}} P_{+},} \\
& {\left[P_{P_{\hat{i}}}, J_{\overline{\hat{j}} \overline{\hat{k}}}\right]=\eta_{\overline{\hat{i}} \overline{\hat{j}}} P_{\hat{\hat{k}}}-\eta_{\overline{\hat{i}} \hat{\bar{k}}} P_{\overline{\hat{j}}}, \quad\left[J_{\overline{\hat{i}} \overline{\hat{j}}}, P_{\hat{\hat{k}}}^{*}\right]=\eta_{\overline{\hat{j}} \overline{\hat{k}}} P_{\hat{i}}^{*}-\eta_{\overline{\hat{i}} \overline{\hat{k}}} P_{\hat{j}}^{*},}
\end{aligned}
$$

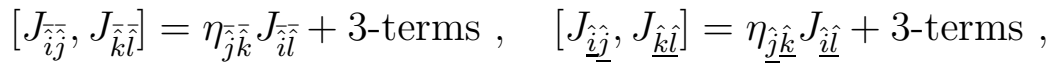

$$
\begin{aligned}
& {\left[Q_{+}^{(-)}, P_{\hat{i}}\right]=-\frac{1}{2 \sqrt{2}} Q_{+}^{(+)} \Gamma_{\overline{\hat{i}}} \Gamma_{+} f i \sigma_{2}, \quad\left[Q_{+}^{(+)}, P_{-}\right]=-\frac{1}{\sqrt{2}} Q_{+}^{(+)} f i \sigma_{2},} \\
& {\left[Q_{+}^{(-)}, P_{\hat{i}}^{*}\right]=\frac{1}{2 \sqrt{2}} Q_{+}^{(+)} \Gamma_{+} \Gamma_{\overline{\hat{i}}}, \quad\left[Q_{+}^{(\bullet)}, J_{\overline{\hat{i}} \overline{\hat{j}}}\right]=-\frac{1}{2} Q_{+}^{(\bullet)} \Gamma_{\overline{\hat{i}} \overline{\hat{j}}}, \quad\left[Q_{+}^{(\bullet)}, J_{\underline{\hat{i}} \hat{j}}\right]=-\frac{1}{2} Q_{+}^{(\bullet)} \Gamma_{\underline{\hat{i}} \underline{\hat{j}}},} \\
& \left\{Q_{+}^{(+)}, Q_{+}^{(+)}\right\}=2 i \mathcal{C} \Gamma_{-} \mathcal{P}_{+} P_{+} \text {, } \\
& \left\{Q_{+}^{(-)}, Q_{+}^{(-)}\right\}=2 i \mathcal{C} \Gamma_{+} \mathcal{P}_{-} P_{+}-i \frac{\lambda}{\sqrt{2}} \mathcal{C} \widehat{\Gamma}^{\overline{\hat{i}} \overline{\hat{j}}} i \sigma_{2} \ell_{-} \mathcal{P}_{+} J_{\overline{\hat{i}} \overline{\hat{j}}}-i \frac{\lambda}{\sqrt{2}} \mathcal{C} \widehat{\Gamma} \hat{\underline{\hat{i}} \hat{\hat{j}}} i \sigma_{2} \ell_{-} \mathcal{P}_{+} J_{\hat{\underline{i}} \hat{\underline{j}}}, \\
& \left\{Q_{+}^{(+)}, Q_{+}^{(-)}\right\}=2 i \mathcal{C} \Gamma^{\overline{\hat{i}}} \ell_{-} \mathcal{P}_{+} P_{\hat{i}}+i \lambda \mathcal{C} \widehat{\Gamma}^{\overline{\hat{i}}} i \sigma_{2} \ell_{-} \mathcal{P}_{+} P_{\hat{\bar{i}}}^{*} \text {. }
\end{aligned}
$$


This is regarded as a supersymmetrization of the pp-wave algebra which is the isometry on the brane worldvolume and the Lorentz symmetry in the transverse space. The existence of this super-subalgebra is ensured by the conditions (3.11) and (3.12).

\subsection{Euclidean branes}

We consider the case that $(+,-)$ are contained in the Dirichlet direction. Let us denote Neumann and Dirichlet directions as $\bar{A}=\overline{\hat{i}}$ and $\underline{A}=(+,-, \underline{\hat{i}})$, respectively. We derive the NH superalgebra of a Euclidean pp-wave brane as an IW contraction of the pp-wave superalgebra.

First we consider the bosonic subalgebra. We rescale generators in the pp-wave algebra as

$$
P_{\underline{A}} \rightarrow \frac{1}{\Omega} P_{\underline{A}}, \quad J_{\underline{\hat{i} \hat{j}}} \rightarrow \frac{1}{\Omega} J_{\underline{\hat{i} \hat{j}}}, \quad P_{\hat{\hat{i}}}^{*} \rightarrow \frac{1}{\Omega} P_{\hat{i}}^{*},
$$

and then take the limit $\Omega \rightarrow 0$. Under the contraction, we obtain the NH algebra of a Euclidean pp-wave brane

$$
\begin{aligned}
& {\left[P_{-}, P_{\hat{\hat{i}}}\right]=-\frac{\lambda^{2}}{\sqrt{2}} P_{\hat{\hat{i}}}^{*}, \quad\left[P_{-}, P_{\underline{\hat{i}}}^{*}\right]=\frac{1}{\sqrt{2}} P_{\underline{\hat{i}}}, \quad\left[P_{\overline{\hat{i}}}, J_{\overline{\hat{j}} \underline{\hat{k}}}\right]=\eta_{\overline{\hat{i}} \overline{\hat{j}}} P_{\underline{\hat{k}}}, \quad\left[J_{\overline{\hat{i}} \underline{\hat{j}}}, P_{\underline{\hat{k}}}^{*}\right]=\eta_{\underline{\hat{j}} \underline{\hat{k}}} P_{\hat{\hat{i}}}^{*},} \\
& {\left[P_{\hat{i}}, P_{\hat{\hat{j}}}^{*}\right]=-\frac{1}{\sqrt{2}} \eta_{\hat{i} \overline{\hat{j}}} P_{+}, \quad\left[P_{\underline{\hat{i}}}, P_{\underline{\hat{j}}}^{*}\right]=-\frac{1}{\sqrt{2}} \eta_{\underline{\hat{i}} \underline{\hat{j}}} P_{+},}
\end{aligned}
$$

and (3.7).

To contract the fermionic part of the pp-wave superalgebra, we introduce a matrix

$$
M=\ell \Gamma^{\bar{A}_{1} \cdots \bar{A}_{p}} \rho, \quad \ell^{2}(-1)^{\left[\frac{p+1}{2}\right]} \rho^{2}=1
$$

and decompose $Q^{( \pm)}$as

$$
Q_{ \pm}^{(\bullet)}= \pm Q_{ \pm}^{(\bullet)} M
$$

where $p=$ odd for the chirality of $Q^{( \pm)}$. We demand that (3.11) and (3.12) are satisfied. The first condition (3.11) is satisfied when

$$
\pm(-1)^{p+\left[\frac{p+1}{2}\right]}=1, \quad \rho^{T}= \pm \rho
$$

so that $\rho^{T}=\rho$ for $p=1 \bmod 4$ and $\rho^{T}=-\rho$ for $p=3 \bmod 4$. It follows that $M^{\prime}=-M$ and $\ell=\sqrt{-1}$. Next, the second condition (3.12) is found to be satisfied when

$$
\pm(-1)^{p+\mathrm{n}}=1, \quad \rho=\left\{\begin{array}{l}
1, i \sigma_{2} \\
\sigma_{1}, \sigma_{3}
\end{array} .\right.
$$


This restricts the brane configuration as follows: (odd,odd)-branes with $\rho=1$ and (even,even)-branes with $\rho=\sigma_{1}, \sigma_{3}$ for $p=1 \bmod 4$, and (odd,odd)-branes with $\rho=i \sigma_{2}$ for $p=3 \bmod 4$. We summarize the result in Table 3. (even,even)-branes of $p=1 \bmod 4$

\begin{tabular}{|c|c|c|c|c|c|}
\hline$\rho$ & 1-brane & 3-brane & 5-brane & 7-brane & 9-brane \\
\hline$\sigma_{1}, \sigma_{3}$ & $(0,2),(2,0)$ & & $(2,4),(4,2)$ & & - \\
\hline$i \sigma_{2}$ & & $(1,3),(3,1)$ & & - & \\
\hline 1 & $(1,1)$ & & $(1,3),(3,1)$ & & $(5,5)$ \\
\hline
\end{tabular}

Table 3: Euclidean pp-wave branes

and (odd,odd)-branes of $p=3 \bmod 4$ are $1 / 2$ BPS D-branes of an open pp-wave superstring $[16,30]$.

Scaling $Q_{ \pm}^{(\bullet)}$ as (3.17) and taking the limit $\Omega \rightarrow 0$, we obtain the fermionic part of the NH superalgebra of a Euclidean pp-wave brane

$$
\begin{aligned}
& {\left[Q_{ \pm}^{(-)}, P_{\hat{\hat{i}}}\right]=-\frac{1}{2 \sqrt{2}} Q_{ \pm}^{(+)} \Gamma_{\overline{\hat{i}}} \Gamma_{+} f i \sigma_{2}, \quad\left[Q_{+}^{(-)}, P_{\underline{\hat{i}}}\right]=-\frac{1}{2 \sqrt{2}} Q_{\mp}^{(+)} \Gamma_{\underline{\hat{i}}} \Gamma_{+} f i \sigma_{2}} \\
& {\left[Q_{+}^{(+)}, P_{-}\right]=-\frac{1}{\sqrt{2}} Q_{-}^{(+)} f i \sigma_{2}, \quad\left[Q_{+}^{(-)}, P_{\hat{i}}^{*}\right]=\frac{1}{2 \sqrt{2}} Q_{-}^{(+)} \Gamma_{+} \Gamma_{\overline{\hat{i}}},} \\
& {\left[Q_{ \pm}^{(-)}, P_{\underline{\hat{i}}}^{*}\right]=\frac{1}{2 \sqrt{2}} Q_{ \pm}^{(+)} \Gamma_{+} \Gamma_{\underline{\hat{i}}}, \quad\left[Q_{ \pm}^{(\bullet)}, J_{\overline{\hat{i}} \bar{j}}\right]=-\frac{1}{2} Q_{ \pm}^{(\bullet)} \Gamma_{\overline{\hat{i}} \overline{\hat{j}}}, \quad\left[Q_{ \pm}^{(\bullet)}, J_{\hat{\underline{\hat{j}}} \underline{j}}\right]=-\frac{1}{2} Q_{ \pm}^{(\bullet)} \Gamma_{\underline{\hat{i} \hat{j}}}} \\
& {\left[Q_{+}^{(\bullet)}, J_{\hat{\hat{i} \hat{j}} \underline{j}}=-\frac{1}{2} Q_{-}^{(\bullet)} \Gamma_{\overline{\hat{i}} \underline{\hat{j}}},\right.} \\
& \left\{Q_{ \pm}^{(+)}, Q_{\mp}^{(+)}\right\}=2 i \mathcal{C} \Gamma_{-} P_{+} \\
& \left\{Q_{ \pm}^{(-)}, Q_{\mp}^{(-)}\right\}=2 i \mathcal{C} \Gamma_{+} P_{-}-2 i \frac{\lambda}{\sqrt{2}} \mathcal{C} \widehat{\Gamma}^{\overline{\bar{i}} \underline{\hat{j}}} i \sigma_{2} J_{\overline{\hat{\bar{i}}} \underline{\hat{j}}}, \\
& \left\{Q_{+}^{(-)}, Q_{+}^{(-)}\right\}=-i \frac{\lambda}{\sqrt{2}} \mathcal{C} \widehat{\Gamma}^{\overline{\hat{i}} \overline{\hat{j}}} i \sigma_{2} J_{\overline{\hat{i}} \overline{\hat{j}}}-i \frac{\lambda}{\sqrt{2}} \mathcal{C} \widehat{\Gamma}^{\hat{i} \hat{j}} i \sigma_{2} J_{\underline{\hat{i}} \hat{j}}, \\
& \left\{Q_{+}^{( \pm)}, Q_{+}^{(\mp)}\right\}=2 i \mathcal{C} \Gamma^{\overline{\hat{i}}} \ell_{\mp} P_{\hat{i}}+i \lambda \mathcal{C} \widehat{\Gamma}^{\hat{i}} \ell_{\mp} i \sigma_{2} P_{\underline{\hat{i}}}^{*}, \\
& \left\{Q_{+}^{( \pm)}, Q_{-}^{(\mp)}\right\}=2 i \mathcal{C} \Gamma^{\hat{i}} \ell_{\mp} P_{\underline{\hat{i}}}+i \lambda \mathcal{C} \widehat{\Gamma}^{\overline{\hat{i}}} \ell_{\mp} i \sigma_{2} P_{\hat{i}}^{*} \text {. }
\end{aligned}
$$

Summarizing we have obtained the NH superalgebra of Euclidean pp-wave brane as (3.21), (3.7) and (3.26). Obviously, this superalgebra can be derived from the NH superalgebra of an AdS brane (2.9) and (2.18) by an IW contraction.

We note that the NH superalgebra contains a super-subalgebra generated by $P_{\hat{i}}, P_{\underline{\hat{i}}}^{*}$, 
$J_{\overline{\hat{i}} \bar{j}}, J_{\underline{\hat{i} \hat{j}}}$ and $Q_{+}^{( \pm)}$

$$
\begin{aligned}
& {\left[P_{\hat{i}}, J_{\overline{\hat{j}} \hat{\hat{k}}}\right]=\eta_{\overline{\hat{i}} \overline{\hat{j}}} P_{\hat{\hat{k}}}-\eta_{\overline{\hat{i}} \overline{\hat{k}}} P_{\overline{\hat{j}}}, \quad\left[J_{\underline{\hat{i} \hat{j}}}, P_{\underline{\hat{k}}}^{*}\right]=-\eta_{\underline{\hat{i}} \underline{\hat{k}}} P_{\underline{\hat{j}}}^{*}+\eta_{\underline{\hat{j} \hat{k}} \underline{\hat{k}}} P_{\underline{\hat{i}}}^{*},} \\
& {\left[J_{\overline{\hat{i}} \overline{\hat{j}}}, J_{\overline{\hat{k}} \hat{\hat{l}}}\right]=\eta_{\overline{\hat{j}} \overline{\hat{k}}} J_{\overline{\hat{i}} \overline{\hat{l}}}+3 \text {-terms }, \quad\left[J_{\underline{\hat{i}} \underline{\hat{j}}}, J_{\underline{\hat{k} \hat{l}}}\right]=\eta_{\underline{\hat{j}} \underline{\hat{k}}} J_{\underline{\hat{i} \hat{l}}}+3 \text {-terms }} \\
& {\left[Q_{+}^{(-)}, P_{\hat{i}}\right]=-\frac{1}{2 \sqrt{2}} Q_{+}^{(+)} \Gamma_{\overline{\hat{i}}} \Gamma_{+} f i \sigma_{2}, \quad\left[Q_{+}^{(-)}, P_{\underline{\hat{i}}}^{*}\right]=\frac{1}{2 \sqrt{2}} Q_{+}^{(+)} \Gamma_{+} \Gamma_{\underline{\hat{i}}},} \\
& {\left[Q_{+}^{(\bullet)}, J_{\overline{\hat{i}} \overline{\hat{j}}}\right]=-\frac{1}{2} Q_{+}^{(\bullet)} \Gamma_{\overline{\hat{i}} \overline{\hat{j}}}, \quad\left[Q_{+}^{(\bullet)}, J_{\underline{\hat{i}} \underline{j}}\right]=-\frac{1}{2} Q_{+}^{(\bullet)} \Gamma_{\underline{\hat{i} \hat{j}}},}
\end{aligned}
$$

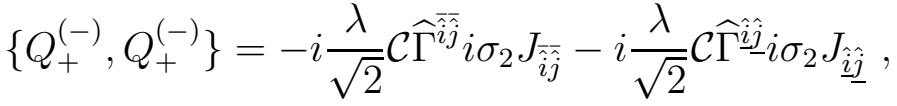

$$
\begin{aligned}
& \left\{Q_{+}^{( \pm)}, Q_{+}^{(\mp)}\right\}=2 i \mathcal{C} \Gamma^{\overline{\hat{i}}} \ell_{\mp} P_{\widehat{\hat{i}}}+i \lambda \mathcal{C} \widehat{\Gamma}^{\hat{i}} \ell_{\mp} i \sigma_{2} P_{\underline{\hat{i}}}^{*}
\end{aligned}
$$

which is regarded as a supersymmetrization of the Poincaré algebra generated by $\left\{P_{\hat{\hat{i}}}, J_{\overline{\hat{i}} \hat{j}}\right\}$ which is the isometry on the brane worldvolume and the Lorentz symmetry in the transverse space generated by $\left\{P_{\underline{\hat{i}}}^{*}, J_{\underline{\hat{i}} \hat{j}}\right\}$. The conditions (3.11) and (3.12) ensure the existence of this super-subalgebra.

\section{Branes in $\operatorname{AdS}_{5} \times \mathbf{S}^{5}$}

A D-brane action [36] (see [37] for flat D-branes) is composed of the Dirac-Born-Infeld (DBI) action and the WZ action

$$
S=S_{\mathrm{DBI}}+S_{\mathrm{WZ}}
$$

The DBI action is given, suppressing the dilaton and axion factors here, as

$$
S_{\mathrm{DBI}}=T \int_{\Sigma} \mathcal{L}_{\mathrm{DBI}}, \quad \mathcal{L}_{\mathrm{DBI}}=\sqrt{s \operatorname{det}(g+\mathcal{F})} d^{p+1} \xi
$$

where $\mathcal{F}=F-\mathcal{B}$ and $F=d A$, and $s=-1$ for a Lorentzian brane while $s=1$ for a Euclidean brane. $T$ is the tension of the brane. $\mathcal{B}$ is the pullback of the NS-NS two-form and $A$ is the gauge field on the worldvolume. For an F-string, the DBI action is replaced by the Nambu-Goto (NG) action

$$
S_{\mathrm{NG}}=T \int_{\Sigma} \mathcal{L}_{\mathrm{NG}}, \quad \mathcal{L}_{\mathrm{NG}}=\sqrt{s \operatorname{det} g} d^{2} \xi .
$$

The WZ action ${ }^{2}$ is characterized by supersymmetric closed $(p+2)$-form $h_{p+2}$

$$
S_{\mathrm{WZ}}=T \int_{\Sigma} \mathcal{L}_{\mathrm{WZ}}, \quad h_{p+2}=d \mathcal{L}_{\mathrm{WZ}}=\sum_{n=0} \frac{1}{n !} h^{(p+2-2 n)} \mathcal{F}^{n} .
$$

\footnotetext{
${ }^{2}$ See [38] for the Roiban-Siegel formulation [39] of AdS D-branes.
} 
The closedness of $h_{p+2}$

$$
0=d h_{p+2}=\sum_{n=0} \frac{1}{n !}\left(d h^{(p+2-2 n)}-h^{(p-2 n)} d \mathcal{F}\right) \mathcal{F}^{n}
$$

implies

$$
d h^{(p+2-2 n)}-h^{(p-2 n)} d \mathcal{F}=0 .
$$

\subsection{CE-cohomology classification}

In [20], it is shown that the Wess-Zumino (WZ) terms of $p$-branes in flat spacetime can be classified as non-trivial elements of the Chevalley-Eilenberg (CE) cohomology [21]. Let $C^{p}(\mathfrak{g}, \mathbb{R})$ be the vector space of $p$-cochains of a Lie algebra $\mathfrak{g}$. A $p$-cochain is a linear antisymmetric map: $\mathfrak{g} \times \cdots \times \mathfrak{g} \mapsto \mathbb{R}$ and a coboundary operator $\delta$ with $\delta^{2}=0$ acts as $C^{p}(\mathfrak{g}, \mathbb{R}) \mapsto C^{p+1}(\mathfrak{g}, \mathbb{R})$. The CE cohomology group $H^{p}(\mathfrak{g}, \mathbb{R})$ is defined by $Z^{p} / B^{p}$ where $Z^{p}$ and $B^{p}$ are the vector spaces of $p$-cocycles $c \in Z^{p}$ satisfying $\delta c=0$ and $p$-coboundaries $c \in B^{p}$ satisfying $c=\delta c^{\prime}$ with $c^{\prime} \in C^{p-1}(\mathfrak{g}, \mathbb{R})$, respectively. In the present context, this is viewed as the de Rham cohomology group $E^{p}(G, \mathbb{R})$ for left-invariant (LI) $p$-forms on the supergroup $G=$ "super-Poincaré" / "Lorentz", for which a non-trivial element of the cohomology is a closed LI $p$-form modulo exact LI $p$-forms on $G$. This is generalized to D-branes in $[22,23]$ by introducing an additional two form which corresponds to the modified field strength of background $\mathcal{B}$ field.

Here we examine WZ terms of AdS branes by using the CE cohomology on $\mathfrak{g}$ of the supergroup $G=\mathrm{PSU}(2,2 \mid 4) /(\mathrm{SO}(4,1) \times \mathrm{SO}(5))$, i.e. "super- $\mathrm{AdS}_{5} \times \mathrm{S}^{5}$ " / "Lorentz". We

show that except for the $p=1$ case $h_{p+2}$ can be obtained as a Lorentz invariant non-trivial element of the CE-cohomology on the free differential algebra which is the MC equations (2.6) corresponding to the super- $\mathrm{AdS}_{5} \times \mathrm{S}^{5}$ algebra (2.1) equipped with

$$
d \mathcal{F}=-i \mathbf{L}^{A} \bar{L} \Gamma_{A} \sigma L
$$

where $\sigma$ is $\sigma_{3}$ for D-branes while $-\sigma_{1}$ for F1- and NS5-branes.

In order not to introduce an additional dimensionful parameter we assign a dimension to Cartan one-forms as follows

$$
\begin{aligned}
& \begin{array}{lllllll}
\mathbf{L}^{A} & L^{\alpha} & \mathbf{L}^{A B} & \lambda & \mathcal{F} & h_{p+2} & h^{(k)}
\end{array} \\
& \operatorname{dim} 1 \frac{1 / 2}{2} \quad 0 \quad-12 p+1 k-1
\end{aligned}
$$


where $\operatorname{dim} h_{p+2}=p+1$ because $\operatorname{dim} h_{p+2}=\operatorname{dim} \mathcal{L}_{\mathrm{WZ}}^{p}=\operatorname{dim} \mathcal{L}_{B I}^{p}=p+1$ for structureless fundamental branes.

Suppose that $h^{(k)}$ is of the form $\left(\mathbf{L}^{A}\right)^{n}\left(L^{\alpha}\right)^{m} \lambda^{l}$, then $n, m$ and $l$ must satisfy

$$
n+\frac{1}{2} m-l=k-1, \quad n+m=k,
$$

because $h^{(k)}$ is a Lorentz invariant $k$-form of dimension $k-1$. We require that $\epsilon_{a_{1} \cdots a_{5}}$ and $\epsilon_{a_{1}^{\prime} \cdots a_{5}^{\prime}}$ are accompanied with $\lambda ; \lambda \epsilon_{a_{1} \cdots a_{5}}$ and $\lambda \epsilon_{a_{1}^{\prime} \cdots a_{5}^{\prime}}$, because $\epsilon_{a_{1} \cdots a_{5}}$ and $\epsilon_{a_{1}^{\prime} \cdots a_{5}^{\prime}}$ disappear in the flat limit $\lambda \rightarrow 0$. Requiring $l \geq 0$ because otherwise $h^{(k)}$ diverges in the flat limit. This implies $l=-\frac{1}{2} m+1 \leq 1$ and so we consider $l=0,1$. Since (4.9) is satisfied for $(m, n)=(2, k-2),(0, k)$ for $l=0,1$, respectively, we find that $h^{(k)}, k=1,3,5, \ldots$, has the following form

$$
\begin{aligned}
h^{(1)}= & 0 \\
h^{(3)}= & c_{0}^{(3)} \mathbf{L}^{a} \bar{L} \Gamma_{a} \varrho_{0}^{(3)} L+c_{1}^{(3)} \mathbf{L}^{a^{\prime}} \bar{L} \Gamma_{a^{\prime}} \varrho_{1}^{(3)} L \\
h^{(5)}= & c_{0}^{(5)} \mathbf{L}^{a_{1}} \mathbf{L}^{a_{2}} \mathbf{L}^{a_{3}} \bar{L} \Gamma_{a_{1} a_{2} a_{3}} \varrho_{0}^{(5)} L+\cdots+c_{3}^{(5)} \mathbf{L}^{a_{1}^{\prime}} \mathbf{L}^{a_{2}^{\prime}} \mathbf{L}^{a_{3}^{\prime}} \bar{L} \Gamma_{a_{1}^{\prime} a_{2}^{\prime} a_{3}^{\prime}} \varrho_{3}^{(5)} L \\
& +b_{0} \lambda \epsilon_{a_{1} \cdots a_{5}} \mathbf{L}^{a_{1}} \cdots \mathbf{L}^{a_{5}}+b_{5} \lambda \epsilon_{a_{1}^{\prime} \cdots a_{5}^{\prime}} \mathbf{L}_{1}^{a_{1}^{\prime}} \cdots \mathbf{L}^{a_{5}^{\prime}} \\
h^{(7)}= & c_{0}^{(7)} \mathbf{L}^{a_{1}} \cdots \mathbf{L}^{a_{5}} \bar{L} \Gamma_{a_{1} \cdots a_{5}} \varrho_{0}^{(7)} L+\cdots+c_{5}^{(7)} \mathbf{L}^{a_{1}^{\prime}} \cdots \mathbf{L}^{a_{5}^{\prime}} \bar{L} \Gamma_{a_{1}^{\prime} \cdots a_{5}^{\prime}} \varrho_{5}^{(7)} L, \\
h^{(9)}= & c_{0}^{(9)} \mathbf{L}^{a_{1}} \cdots \mathbf{L}^{a_{7}} \bar{L} \Gamma_{a_{1} \cdots a_{7}} \varrho_{0}^{(9)} L+\cdots+c_{7}^{(9)} \mathbf{L}^{a_{1}^{\prime}} \cdots \mathbf{L}^{a_{7}^{\prime}} \bar{L} \Gamma_{a_{1}^{\prime} \cdots a_{7}^{\prime}} \varrho_{7}^{(9)} L,
\end{aligned}
$$

where $c_{i}^{(k)}$ and $b_{i}$ are constants determined below. $\varrho_{i}^{(k)}$ are $2 \times 2$ matrices satisfying $\varrho^{(k)^{T}}=\varrho^{(k)}$ for $k=3,7$ while $\varrho^{(k)^{T}}=-\varrho^{(k)}$ for $k=5,9$, because $\mathcal{C} \Gamma^{A_{1} \cdots A_{N}}$ is symmetric for $N=1,2 \bmod 4$ and anti-symmetric otherwise.

It is straightforward to solve (4.6) to determine coefficients and $\varrho_{i}^{(k)}$. We find

$$
\begin{aligned}
h^{(1)} & =0 \\
h^{(3)} & =c \mathbf{L}^{A} \bar{L} \Gamma_{A} \varrho L \\
h^{(5)} & =\frac{c}{3 !}\left[\mathbf{L}^{A_{1}} \mathbf{L}^{A_{2}} \mathbf{L}^{A_{3}} \bar{L} \Gamma_{A_{1} A_{2} A_{3}} i \sigma_{2} L+\frac{i}{5} \lambda\left(\epsilon_{a_{1} \cdots a_{5}} \mathbf{L}^{a_{1}} \cdots \mathbf{L}^{a_{5}}-\epsilon_{a_{1}^{\prime} \cdots a_{5}^{\prime}} \mathbf{L}^{a_{1}^{\prime}} \cdots \mathbf{L}^{a_{5}^{\prime}}\right)\right], \\
h^{(7)} & =\frac{c}{5 !} \mathbf{L}^{A_{1}} \cdots \mathbf{L}^{A_{5}} \bar{L} \Gamma_{A_{1} \cdots A_{5}} \varrho L \\
h^{(9)} & =\frac{c}{7 !} \mathbf{L}^{A_{1}} \cdots \mathbf{L}^{A_{7}} \bar{L} \Gamma_{A_{1} \cdots A_{7}} i \sigma_{2} L .
\end{aligned}
$$

In Appendix B, $c=c_{0}^{(3)}$ is determined by the $\kappa$-invariance [36] of the total action $S$ as $c=i$ and 1 for Lorentzian and Euclidean branes respectively: $c=\sqrt{s} . \varrho$ is $\sigma_{1}\left(\sigma_{3}\right)$ for 
$\sigma=\sigma_{3}\left(-\sigma_{1}\right)$ respectively. The closedness (4.6) is ensured by the Fierz identities

$$
\begin{aligned}
& \left(\mathcal{C} \Gamma_{A}\right)_{(\alpha \beta}\left(\mathcal{C} \Gamma^{A} \varrho\right)_{\gamma \delta)}=0 \\
& \left(\mathcal{C} \Gamma_{C}\right)_{(\alpha \beta}\left(\mathcal{C} \Gamma^{A B C} i \sigma_{2}\right)_{\gamma \delta)}+2\left(\mathcal{C} \Gamma^{[A} \varrho\right)_{(\alpha \beta}\left(\mathcal{C} \Gamma^{B]} \sigma\right)_{\gamma \delta)}=0 \\
& \left(\mathcal{C} \Gamma_{B}\right)_{(\alpha \beta}\left(\mathcal{C} \Gamma^{A_{1} \cdots A_{4} B} \varrho\right)_{\gamma \delta)}+4\left(\mathcal{C} \Gamma^{\left[A_{1} A_{2} A_{3}\right.} i \sigma_{2}\right)_{(\alpha \beta}\left(\mathcal{C} \Gamma^{\left.A_{4}\right]} \sigma\right)_{\gamma \delta)}=0 \\
& \left(\mathcal{C} \Gamma_{B}\right)_{(\alpha \beta}\left(\mathcal{C} \Gamma^{A_{1} \cdots A_{6} B} i \sigma_{2}\right)_{\gamma \delta)}+6\left(\mathcal{C} \Gamma^{\left[A_{1} \cdots A_{5}\right.} \varrho\right)_{(\alpha \beta}\left(\mathcal{C} \Gamma^{\left.A_{6}\right]} \sigma\right)_{\gamma \delta)}=0
\end{aligned}
$$

In summary, closed $(p+2)$-forms $h_{p+2}$ are composed in terms of $h^{(k)}$ found above as in (4.4). The actions $S$ for F1- and D3-branes coincide with those obtained in [40] and [41], respectively.

We show that $h_{p+2}$ is a non-trivial element of the cohomology except for $h_{3}$. If $h_{p+2}$ is exact, there exists $b_{p+1}$ such as $h_{p+2}=d b_{p+1}$. Since

$$
h_{3}=d b_{2}, \quad b_{2}=-c \lambda^{-1} \bar{L} \mathcal{I} \varrho i \sigma_{2} L
$$

$h_{3}$ is a trivial element of the cohomology [24,25]. Next we show that $h_{p+2}$ with $p=3,5,7$ is not exact. Let us examine a term of the form $\frac{1}{\left(\frac{p-1}{2}\right) !} h^{(3)} \mathcal{F}^{\frac{p-1}{2}}$ contained in $h_{p+2}$. We note that $\mathcal{F}$ can be written as ${ }^{3}$

$$
\mathcal{F}=i c \lambda^{-1} \bar{L} \mathcal{I} \sigma i \sigma_{2} L
$$

up to an exact form, and that there does not exist a one-form supercurrent $f$ such that $\mathcal{F}=d f$. So $b_{p+1}$ must contain a term of the form $\bar{L} \mathcal{I} \varrho i \sigma_{2} L \mathcal{F}^{\frac{p-1}{2}}$. Differentiating it, we have $\bar{L} \mathcal{I} \varrho i \sigma_{2} L L^{A} \bar{L} \Gamma_{A} \sigma L \mathcal{F}^{\frac{p-3}{2}}$ in addition to $\frac{1}{\left(\frac{p-1}{2}\right) !} h^{(3)} \mathcal{F}^{\frac{p-1}{2}}$. For $h_{p+2}$ to be exact, this term must be canceled by the differential of a term which is a $(p+1)$-form with $p-1$ $L^{\alpha}$ 's. From the MC equation (2.6), we see that there does not exist such a term. Thus $h_{p+2}$ with $p=3,5,7$ obtained above are non-trivial elements of the cohomology.

\section{2 $(p+1)$-dimensional form of the WZ term}

In this subsection, we give the $(p+1)$-dimensional form of the WZ term $h_{p+2}$. We follow $[40,41]$ in which the $(p+1)$-dimensional form of the WZ term of F1- and D3-branes are given.

\footnotetext{
${ }^{3}$ This implies that $h_{2}=h^{(2)}+h^{(0)} \mathcal{F}$ with $h^{(0)}=i \hat{c} \lambda$ and $h^{(2)}=-\hat{c} \bar{L} \mathcal{I} \sigma i \sigma_{2} L$ can be a nontrivial element of the cohomology. It is interesting to examine the 0-brane action with the WZ term $h_{2}$.
} 
The LI Cartan one-forms satisfy the following differential equations

$$
\begin{aligned}
\partial_{t} \hat{\mathbf{L}}^{A} & =2 i \bar{\theta} \Gamma^{A} \hat{L}, \\
\partial_{t} \hat{L}^{\alpha} & =d \theta+\frac{\lambda}{2} \hat{\mathbf{L}}^{A} \widehat{\Gamma}_{A} i \sigma_{2} \theta+\frac{1}{4} \hat{\mathbf{L}}^{A B} \Gamma_{A B} \theta, \\
\partial_{t} \hat{\mathbf{L}}^{A B} & =-2 i \lambda \bar{\theta} \widehat{\Gamma}^{A B} i \sigma_{2} \hat{L}
\end{aligned}
$$

where a "hat" on a supercurrent implies that $\theta$ is rescaled as $\theta \rightarrow t \theta$. First we note that

$$
\partial_{t} d \hat{\mathcal{F}}=-\partial_{t} d \hat{\mathcal{B}}=-2 i d\left(\hat{\mathbf{L}}^{A} \hat{\bar{L}} \Gamma_{A} \sigma \theta\right)
$$

This is solved by

$$
\mathcal{B}=2 i \int_{0}^{1} d t \hat{\mathbf{L}}^{A} \hat{\bar{L}} \Gamma_{A} \sigma \theta+B^{(2)} .
$$

where $B^{(2)}$ is a bosonic 2-form satisfying $d B^{(2)}=0$. Thus we obtain

$$
\begin{aligned}
\mathcal{F} & =F-2 i \int_{0}^{1} d t \hat{\mathbf{L}}^{A} \hat{\bar{L}} \Gamma_{A} \sigma \theta-B^{(2)}, \\
\partial_{t} \hat{\mathcal{F}} & =-2 i\left(\hat{\mathbf{L}}^{A} \hat{\bar{L}} \Gamma_{A} \sigma \theta\right) .
\end{aligned}
$$

For D-brane actions, we choose $B^{(2)}=0$.

By using (4.23)-(4.25) and (4.29), one sees that the closed $(p+1)$-form $h_{p+2}$ satisfies

$$
\partial_{t} \hat{h}_{p+2}=d b_{p+1}
$$

where

$$
\begin{aligned}
b_{p+1} & =\left[\mathcal{C} \wedge \mathrm{e}^{\hat{\mathcal{F}}}\right]_{p+1}, \quad \mathcal{C}=\bigoplus_{\ell=\text { even }} \mathcal{C}^{(\ell)} \\
\mathcal{C}^{(2 n)} & =\frac{2 \sqrt{s}}{(2 n-1) !} \hat{\mathbf{L}}^{A_{1}} \cdots \hat{\mathbf{L}}^{A_{2 n-1}} \hat{\bar{L}} \Gamma_{A_{1} \cdots A_{2 n-1}}(\sigma)^{n} i \sigma_{2} \theta
\end{aligned}
$$

It follows that

$$
\int_{B} h_{p+2}=\int_{\Sigma} \mathcal{L}_{\mathrm{WZ}}=\int_{\Sigma}\left[\int_{0}^{1} d t b_{p+1}+C^{(p+1)}\right]
$$

where $\partial B=\Sigma$, and $C^{(p+1)}$ is a bosonic $(p+1)$-form satisfying

$$
\left.h_{p+2}\right|_{\text {bosonic }}=d C^{(p+1)} .
$$

Letting $p=1$ and $\sigma=-\sigma_{1}$, we reproduce the WZ term of an F-string. 


\section{Non-relativistic Branes in $\operatorname{AdS}_{5} \times \mathbf{S}^{5}$}

In [15], the non-relativistic F-string in $\mathrm{AdS}_{5} \times \mathrm{S}^{5}$ is examined. There the leading contributions of the NG and the WZ parts in the non-relativistic limit cancel each other, and the next-to-leading terms contribute to the non-relativistic F-string action. Thus, in order to extract non-relativistic brane actions, we need to know the next-to-leading order terms in the limit $\Omega \rightarrow 0$. Let us consider the scaling

$$
\begin{aligned}
& X^{\underline{A}} \rightarrow \Omega X^{\underline{A}}, \quad \theta_{-} \rightarrow \Omega \theta_{-}, \\
& T=\Omega^{-2} T_{\mathrm{NR}}, \quad F=\Omega F_{1} .
\end{aligned}
$$

(5.1) is consistent with the scaling (2.8) and (2.17). It is straightforward to see that by substituting (5.1) into the concrete expression of the supercurrents given in Appendix A.1. $\mathbf{L}^{A}$ and $L$ are expanded as

$$
\begin{aligned}
& \mathbf{L}^{\bar{A}}=\sum_{n=0} \Omega^{2 n} \mathbf{L}_{2 n}^{\bar{A}}, \quad \mathbf{L}^{\underline{A}}=\sum_{n=0} \Omega^{2 n+1} \mathbf{L}_{2 n+1}^{\underline{A}}, \\
& L_{+}=\sum_{n=0} \Omega^{2 n} L_{+2 n}, \quad L_{-}=\sum_{n=0} \Omega^{2 n+1} L_{-2 n+1} .
\end{aligned}
$$

Expand $\mathbf{L}^{A B}$ as

$$
\mathbf{L}^{\bar{A} \bar{B}}=\sum_{n=0} \Omega^{2 n} \mathbf{L}_{2 n}^{\bar{A} \bar{B}}, \quad \mathbf{L} \underline{A B}=\sum_{n=0} \Omega^{2 n} \mathbf{L}_{2 n}^{A B}, \quad \mathbf{L}^{\bar{A} \underline{B}}=\sum_{n=0} \Omega^{2 n+1} \mathbf{L}_{2 n+1}^{\bar{A} \underline{B}},
$$

and substitute (5.3) and (5.4) into the MC equation (2.6) for the super- $\mathrm{AdS}_{5} \times \mathrm{S}^{5}$ algebra, then the LI Cartan one-forms $\left\{\mathbf{L}_{0}^{\bar{A}}, \mathbf{L}_{1}^{A}, L_{+0}, L_{-1}, \mathbf{L}_{0}^{\bar{A} \bar{B}}, \mathbf{L}_{0}^{\underline{A B}}, \mathbf{L}_{1}^{\bar{A}} \underline{B}\right\}$ form the MC equations (2.20) $-(2.28)$ for the NH superalgebra. ${ }^{4}$

We consider the non-relativistic limit of the AdS branes obtained in the previous section. In the following subsections, we will show that when we introduce

$$
M=\sqrt{-s} \Gamma^{\bar{A}_{0} \cdots \bar{A}_{p}} \otimes \rho
$$

with $\rho=\sigma_{1}\left(i \sigma_{2}\right)$ for $\mathrm{D} p$-branes with $p=1(3) \bmod 4$, respectively, and with $\rho=\sigma_{3}$ for F1 and NS5, AdS $p$-brane actions admit expansion

$$
S=T_{\mathrm{NR}} \int_{\Sigma}\left[\Omega^{-2}\left(\mathcal{L}_{\substack{\mathrm{NG} \\
\mathrm{NBI}}}^{d i v}+\mathcal{L}_{\mathrm{WZ}}^{d i v}\right)+\mathcal{L}_{\left\{\begin{array}{l}
\mathrm{NG} \\
\mathrm{NBI}
\end{array}\right.}^{f i n}+\mathcal{L}_{\mathrm{WZ}}^{f i n}+O\left(\Omega^{2}\right)\right]
$$

\footnotetext{
${ }^{4}$ As will be seen below, the non-relativistic actions are composed of $\left\{\mathbf{L}_{0}^{\bar{A}}, \mathbf{L}_{2}^{\bar{A}}, \mathbf{L}_{1}^{A}, L_{+0}, L_{+2}, L_{-1}\right\}$. So these actions are not invariant under the NH superalgebra, but under an expanded superalgebra $[24,43]$ (see also $[44,45]$ ) which is a generalization of the IW contraction [4], generated by generators dual to $\left\{\mathbf{L}_{m}^{\bar{A}}, \mathbf{L} \frac{A}{m}, L_{ \pm m}, \mathbf{L}_{m}^{\bar{A} \bar{B}}, \mathbf{L}_{m}^{\frac{A B}{m}}, \mathbf{L}_{m}^{\bar{A}} \underline{B} \mid 0 \leq m \leq 2\right\}$.
} 
For the consistent non-relativistic limit $\Omega \rightarrow 0$, the divergent term $\int\left(\mathcal{L}_{\left\{\begin{array}{l}\mathrm{NG} \\ \mathrm{NBI}\end{array}\right.}^{\text {iv }}+\mathcal{L}_{\mathrm{WZ}}^{\text {div }}\right)$ should cancel out. First, we show that

$$
d \mathcal{L}_{\left\{\begin{array}{l}
\mathrm{NG} \\
\mathrm{DBI}
\end{array}\right.}^{d i v}+h_{p+2}^{d i v}=0
$$

This implies that the divergent terms with $\theta$ cancel out, since $h_{p+2}^{d i v}$ is composed of only

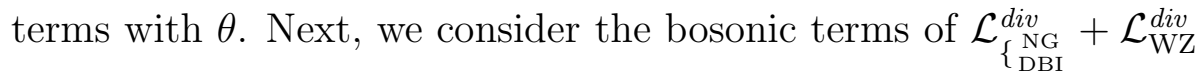

$$
\frac{1}{(p+1) !} \epsilon_{\bar{A}_{0} \cdots \bar{A}_{p}} e_{0}^{\bar{A}_{0}} \cdots e_{0}^{\bar{A}_{p}}+C_{0}^{(p+1)}
$$

where $C_{0}^{(p+1)}$ is the leading contribution of $C^{(p+1)}$ in (4.32). This is deleted by choosing $C_{0}^{(p+1)}=-\frac{1}{(p+1) !} \epsilon_{\bar{A}_{0} \cdots \bar{A}_{p}} e_{0}^{\bar{A}_{0}} \cdots e_{0}^{\bar{A}_{p}}$. It is easy to see that $d C_{0}^{(p+1)}=0$ by using the expressions given in Appendix A.1. Thus the bosonic divergent terms also cancel out. As a result, we derive the non-relativistic brane action

$$
S_{\mathrm{NR}}=T_{\mathrm{NR}} \int_{\Sigma} \mathcal{L}_{\mathrm{NR}}, \quad \mathcal{L}_{\mathrm{NR}}=\mathcal{L}_{\{\mathrm{DBI}}^{f i n}+\mathcal{L}_{\mathrm{WZ}}^{\text {fin }}
$$

which is drastically simplified by gauge fixing the $\kappa$-symmetry by $\theta_{+}=0$. We examine each AdS branes in turn below.

\section{$5.1 \quad$ F-string}

First, we consider an F-string. The 3 -form $h_{3}$ is given in (4.16) with $\varrho=\sigma_{3}$. The gluing matrix $M$ is

$$
M=\sqrt{-s} \Gamma^{\bar{A}_{0} \bar{A}_{1}} \otimes \rho, \quad \rho=\sigma_{1}, \sigma_{3}, 1
$$

Since

$$
M^{\prime} \Gamma_{\bar{A}} \varrho=\Gamma_{\bar{A}} M \varrho= \pm \Gamma_{\bar{A}} \varrho M, \quad \rho=\left\{\begin{array}{l}
\sigma_{3}, 1 \\
\sigma_{1}
\end{array}\right.
$$

$h_{3}$ is expanded as

$$
\begin{aligned}
T h_{3}= & T_{\mathrm{NR}} \Omega^{-2} h_{3}^{d i v}+T_{\mathrm{NR}} h_{3}^{f i n}+O\left(\Omega^{2}\right), \\
h_{3}^{d i v}= & \sqrt{s} \mathbf{L}_{0}^{\bar{A}} \bar{L}_{+0} \Gamma_{\bar{A}} \varrho L_{+0}, \\
h_{3}^{f i n}= & \sqrt{s}\left[\mathbf{L}_{0}^{\bar{A}} \bar{L}_{-1} \Gamma_{\bar{A}} \varrho L_{-1}+\mathbf{L}_{2}^{\bar{A}} \bar{L}_{+0} \Gamma_{\bar{A}} \varrho L_{+0}\right. \\
& \left.+2 \mathbf{L}_{0}^{\bar{A}} \bar{L}_{+0} \Gamma_{\bar{A}} \varrho L_{+2}+2 \mathbf{L}_{1} \bar{L}_{+0} \Gamma_{\underline{A}} \varrho L_{-1}\right],
\end{aligned}
$$


for $\rho=\sigma_{3}, 1$, while $h_{3}$ is of order $\Omega$ for $\rho=\sigma_{1}$. On the other hand, the NG part is expanded as

$$
\begin{aligned}
T \mathcal{L}_{\mathrm{NG}} & =T_{\mathrm{NR}} \Omega^{-2} \mathcal{L}_{\mathrm{NG}}^{d i v}+T_{\mathrm{NR}} \mathcal{L}_{\mathrm{NG}}^{f i n}+O\left(\Omega^{2}\right), \\
\mathcal{L}_{\mathrm{NG}}^{d i v} & =\sqrt{s \operatorname{det} g_{0}} d^{2} \xi=\operatorname{det}\left(\left(\mathbf{L}_{0}^{\bar{A}}\right)_{i}\right) d^{2} \xi=\frac{1}{2} \epsilon_{\bar{A} \bar{B}}\left(\mathbf{L}_{0}^{\bar{A}}\right)\left(\mathbf{L}_{0}^{\bar{B}}\right), \\
\mathcal{L}_{\mathrm{NG}}^{f i n} & =\frac{1}{2} \sqrt{s \operatorname{det} g_{0}} g_{0}^{i j}\left(g_{2}\right)_{i j} d^{2} \xi
\end{aligned}
$$

with $\epsilon_{\bar{A}_{0} \bar{A}_{1}}=1$ and

$$
\begin{aligned}
\left(g_{0}\right)_{i j} & =\left(\mathbf{L}_{0}^{\bar{A}}\right)_{i}\left(\mathbf{L}_{0}^{\bar{B}}\right)_{j} \eta_{\bar{A} \bar{B}}, \\
\left(g_{2}\right)_{i j} & =2\left(\mathbf{L}_{0}^{\bar{A}}\right)_{(i}\left(\mathbf{L}_{2}^{\bar{B}}\right)_{j)} \eta_{\bar{A} \bar{B}}+\left(\mathbf{L}_{1}^{\underline{A}}\right)_{i}\left(\mathbf{L}_{1}^{\underline{B}}\right)_{j} \eta_{\underline{A B}} .
\end{aligned}
$$

The leading contribution satisfies [15]

$$
d \mathcal{L}_{\mathrm{NG}}^{\text {div }}=\epsilon_{\bar{A} \bar{B}} i \bar{L}_{+0} \Gamma^{\bar{A}} L_{+0} \mathbf{L}_{0}^{\bar{B}}=-\sqrt{s} \mathbf{L}_{0}^{\bar{A}} \bar{L}_{+0} \Gamma_{\bar{A}} \rho L_{+0}
$$

where we have used (2.20) and $L_{+}=M L_{+}$. This cancels out $h_{3}^{\text {div }}$ in (5.13) only when $\varrho=\rho$

$$
d \mathcal{L}_{\mathrm{NG}}^{d i v}+h_{3}^{d i v}=0
$$

This implies that $\theta$-dependent terms in $\mathcal{L}_{\mathrm{NG}}^{\text {div }}+\mathcal{L}_{\mathrm{WZ}}^{\text {div }}$ cancel each other. The bosonic term of $\mathcal{L}_{\mathrm{NG}}^{\text {div }}$ in (5.16),$\frac{1}{2} \epsilon_{\bar{A} \bar{B}} e_{0}^{\bar{A}} e_{0}^{\bar{B}}$, is deleted by choosing $C_{0}^{(2)}$ in (4.32) as

$$
C_{0}^{(2)}=-\frac{1}{2} \epsilon_{\bar{A} \bar{B}} e_{0}^{\bar{A}} e_{0}^{\bar{B}}
$$

which satisfies $d C_{0}^{(2)}=0$. Thus, the gluing matrix (5.10) with $\rho=\sigma_{3}$ leads to the consistent non-relativistic limit of the F-string. The non-relativistic F-string action is (5.9) with (5.17) and

$$
\begin{aligned}
\mathcal{L}_{\mathrm{WZ}}^{f i n}= & \int_{0}^{1} d t 2 \sqrt{s}\left[\hat{\mathbf{L}}_{0}^{\bar{A}}\left(\hat{\bar{L}}_{-1} \Gamma_{\bar{A}} \varrho \theta_{-}+\hat{\bar{L}}_{+2} \Gamma_{\bar{A}} \varrho \theta_{+}\right)\right. \\
& \left.+\hat{\mathbf{L}}_{1}^{A}\left(\hat{\bar{L}}_{-1} \Gamma_{\underline{A}} \varrho \theta_{+}+\hat{\bar{L}}_{+0} \Gamma_{\underline{A}} \varrho \theta_{-}\right)+\hat{\mathbf{L}}_{2}^{\bar{A}} \hat{\bar{L}}_{+0} \Gamma_{\bar{A}} \varrho \theta_{+}\right] .
\end{aligned}
$$

We fix the $\kappa$-gauge symmetry of the action by $\theta_{+}=0$ (see Appendix $\mathbb{B}$ ). Then we have

$$
\begin{aligned}
& \mathbf{L}_{0}^{\bar{A}}=e_{0}^{\bar{A}}, \quad \mathbf{L}_{2}^{\bar{A}}=e_{2}^{\bar{A}}+i \bar{\theta}_{-} \Gamma^{\bar{A}} \mathrm{D} \theta_{-}, \quad \mathbf{L}_{1}^{\frac{A}{1}}=e^{\frac{A}{1}}, \\
& L_{-1}=\mathrm{D} \theta_{-}, \quad \mathrm{D} \theta_{-} \equiv d \theta_{-}+\frac{\lambda}{2} e_{0}^{\bar{A}} \widehat{\Gamma}_{\bar{A}} i \sigma_{2} \theta_{-}+\frac{1}{4} \omega_{0}^{\bar{A} \bar{B}} \Gamma_{\bar{A} \bar{B}} \theta_{-}, \\
& \left(g_{0}\right)_{i j}=\left(e_{0}^{\bar{A}}\right)_{i}\left(e_{0}^{\bar{B}}\right)_{j} \eta_{\bar{A} \bar{B}} .
\end{aligned}
$$


In the static gauge, $x^{\bar{A}}=\xi^{i},\left(e_{0}^{\bar{A}}\right)_{i}$ is the vielbein on the AdS brane worldvolume. Thanks to the $\kappa$-gauge fixing, we can perform the $t$-integration in $(5.23)$ easily. $\mathcal{L}_{\mathrm{NG}}^{f i n}$ is reduced to

$$
\mathcal{L}_{\mathrm{NG}}^{f i n}=d^{2} \xi \sqrt{s \operatorname{det} g_{0}}\left[g_{0}^{i j}\left(e_{0}^{\bar{A}}\right)_{i}\left(e_{2}^{\bar{B}}\right)_{j} \eta_{\bar{A} \bar{B}}+\frac{1}{2} g_{0}^{i j}\left(e_{1}^{\frac{A}{1}}\right)_{i}\left(e_{1}^{\underline{B}}\right)_{j} \eta_{\bar{A} \bar{B}}+i g_{0}^{i j} \bar{\theta}_{-} \gamma_{i} \mathrm{D}_{j} \theta_{-}\right]
$$

where $\gamma_{i}=\left(e_{0}^{\bar{A}}\right)_{i} \Gamma_{\bar{A}}$. By parameterizing the group manifold as in Appendix A.1. it is rewritten as

$$
\mathcal{L}_{\mathrm{NG}}^{f i n}=d^{2} \xi \sqrt{s \operatorname{det} g_{0}}\left[\frac{1}{2} g_{0}^{i j} \partial_{i} y^{\underline{A}} \partial_{j} y^{\underline{B}} \eta_{\underline{A B}}+\frac{\lambda^{2}}{2}\left(m y^{2}-n y^{\prime 2}\right)+i \bar{\theta}_{-} \gamma^{i} \mathrm{D}_{i} \theta_{-}\right]
$$

for an $(m, n)$-brane with $(m, n)=(2,0),(0,2)$. On the other hand, $\mathcal{L}_{\text {WZ }}^{\text {in }}$ is reduced to

$$
\mathcal{L}_{\mathrm{WZ}}^{f i n}=\sqrt{s} e_{0}^{\bar{A}} \mathrm{D} \bar{\theta}_{-} \Gamma_{\bar{A}} \varrho \theta_{-}=d^{2} \xi \sqrt{s \operatorname{det} g_{0}}\left[-i \mathrm{D}_{i} \bar{\theta}_{-} \gamma^{i} \theta_{-}\right]
$$

where we have used $\theta_{-}=-M \theta_{-}$in the second equality. Combining these results, we obtain the non-relativistic action

$$
S_{\mathrm{NR}}^{F 1}=T_{\mathrm{NR}} \int d^{2} \xi \sqrt{s \operatorname{det} g_{0}}\left[\frac{1}{2} g_{0}^{i j} \partial_{i} y^{\underline{A}} \partial_{j} y_{\underline{A}}+\frac{\lambda^{2}}{2}\left(m y^{2}-n y^{\prime 2}\right)+2 i \bar{\theta}_{-} \gamma^{i} \mathrm{D}_{i} \theta_{-}\right]
$$

This is a free field action of scalars and fermions propagating on (2,0)- or (0,2)-brane worldvolume. For the case of a Lorentzian $(2,0)$-brane, this reproduces the non-relativistic $\mathrm{AdS}_{2}$ brane action obtained in [15].

\section{$5.2 \quad$ D-string}

Secondly, we consider a D-string, for which $\varrho=\sigma_{1}$ and $\sigma=\sigma_{3}$. The gluing matrix $M$ is given in (15.10). Since

$$
M^{\prime} \Gamma_{\bar{A}} \varrho=\Gamma_{\bar{A}} M \varrho= \pm \Gamma_{\bar{A}} \varrho M, \quad \rho=\left\{\begin{array}{l}
\sigma_{1}, 1 \\
\sigma_{3}
\end{array},\right.
$$

$h_{3}$ is expanded as (5.12) with $\varrho=\sigma_{1}$ for $\rho=\sigma_{1}, 1$, while $h_{3}$ is of order $\Omega$ for $\rho=\sigma_{3}$. We note that for $\rho=\sigma_{1}, \mathcal{F}$ is of order $\Omega$

$$
\begin{aligned}
\mathcal{F} & =\Omega \mathcal{F}_{1}+O\left(\Omega^{3}\right) \\
\mathcal{F}_{1} & =F_{1}-2 i \int_{0}^{1} d t\left[\hat{\mathbf{L}}_{0}^{\bar{A}}\left(\hat{\bar{L}}_{+0} \Gamma_{\bar{A}} \sigma \theta_{-}+\hat{\bar{L}}_{-1} \Gamma_{\bar{A}} \sigma \theta_{+}\right)+\hat{\mathbf{L}}_{1}^{\underline{A}} \hat{\bar{L}}_{+0} \Gamma_{\underline{A}} \sigma \theta_{+}\right]
\end{aligned}
$$

since

$$
M^{\prime} \Gamma_{\bar{A}} \sigma=-\Gamma_{\bar{A}} \sigma M
$$


So, the DBI part is expanded as

$$
\begin{aligned}
T \mathcal{L}_{\mathrm{DBI}} & =T_{\mathrm{NR}} \Omega^{-2} \mathcal{L}_{\mathrm{DBI}}^{d i v}+T_{\mathrm{NR}} \mathcal{L}_{\mathrm{DBI}}^{f i n}+O\left(\Omega^{4}\right) \\
\mathcal{L}_{\mathrm{DBI}}^{d i v} & =\sqrt{s \operatorname{det} g_{0}} d^{2} \xi \\
\mathcal{L}_{\mathrm{DBI}}^{\text {fin }} & =\frac{1}{2} \sqrt{s \operatorname{det} g_{0}}\left(g_{0}^{i j}\left(g_{2}\right)_{i j}-\frac{1}{2} g_{0}^{i k}\left(\mathcal{F}_{1}\right)_{k j} g_{0}^{j l}\left(\mathcal{F}_{1}\right)_{l i}\right) d^{2} \xi
\end{aligned}
$$

where $g_{0}, g_{2}$ and $\mathcal{F}_{1}$ are given in (5.18), (5.19) and (5.31), respectively. For $\rho=1, \sigma_{3}$, $\mathcal{F}$ is of order $\Omega^{0}$. As was done for the F-string case, the $h_{3}^{\text {div }}$ in (5.13) with $\varrho=\sigma_{1}$ and the leading contribution of the fermionic part of the DBI action cancel each other. By choosing $C_{0}^{(2)}=-\frac{1}{2} \epsilon_{\bar{A} \bar{B}} e_{0}^{\bar{A}} e_{0}^{\bar{B}}$, the bosonic terms of the divergent part cancel out. Thus, the gluing matrix with $\rho=\sigma_{1}$ leads to the consistent non-relativistic limit of the D-string.

The non-relativistic D-string action is given by (5.9) with (5.35) and

$$
\begin{aligned}
\mathcal{L}_{\mathrm{WZ}}^{f i n}= & \int_{0}^{1} d t 2 \sqrt{s}\left[\hat{\mathbf{L}}_{0}^{\bar{A}}\left(\hat{\bar{L}}_{-1} \Gamma_{\bar{A}} \varrho \theta_{-}+\hat{\bar{L}}_{+2} \Gamma_{\bar{A}} \varrho \theta_{+}\right)\right. \\
& \left.+\hat{\mathbf{L}}_{1}^{A}\left(\hat{\bar{L}}_{-1} \Gamma_{\underline{A}} \varrho \theta_{+}+\hat{\bar{L}}_{+0} \Gamma_{\underline{A}} \varrho \theta_{-}\right)+\hat{\mathbf{L}}_{2}^{\bar{A}} \hat{\bar{L}}_{+0} \Gamma_{\bar{A}} \varrho \theta_{+}\right] .
\end{aligned}
$$

Let us gauge fix the $\kappa$-gauge symmetry by choosing $\theta_{+}=0$. This makes it easy to perform the $t$-integration in $\mathcal{L}_{\mathrm{WZ}}^{\text {fin }}$. The $t$-integration in $\mathcal{F}_{1}$ in (5.31) disappears and we have $\mathcal{F}_{1}=F_{1}$. In the similar way in the F-string case, we obtain the non-relativistic D-string action

$$
\begin{aligned}
S_{\mathrm{NR}}^{D 1}= & T_{\mathrm{NR}} \int d^{2} \xi \sqrt{s \operatorname{det} g_{0}}\left[\frac{1}{2} g_{0}^{i j} \partial_{i} y^{\underline{A}} \partial_{j} y_{\underline{A}}+\frac{\lambda^{2}}{2}\left(m y^{2}-n y^{\prime 2}\right)\right. \\
& \left.+2 i \bar{\theta}_{-} \gamma^{i} \mathrm{D}_{i} \theta_{-}+\frac{1}{4}\left(F_{1}\right)_{i j}\left(F_{1}\right)^{i j}\right] .
\end{aligned}
$$

This is a free field action of scalars, fermions and a gauge field propagating on $(2,0)$ - or (0,2)-brane worldvolume.

\subsection{D3-brane}

Thirdly, we consider a D3-brane for which $\varrho=\sigma_{1}$ and $\sigma=\sigma_{3}$. The gluing matrix is

$$
M=\sqrt{-s} \Gamma^{\bar{A}_{0} \cdots \bar{A}_{3}} \otimes i \sigma_{2}
$$

Since

$$
M^{\prime} \Gamma_{\bar{B}_{1} \cdots \bar{B}_{3}} i \sigma_{2}=\Gamma_{\bar{B}_{1} \cdots \bar{B}_{3}} i \sigma_{2} M, \quad M^{\prime} \Gamma_{\bar{A}} \varrho=-\Gamma_{\bar{A}} \varrho M, \quad M^{\prime} \Gamma_{\bar{A}} \sigma=-\Gamma_{\bar{A}} \sigma M
$$


$\mathcal{F}$ and $h_{3}$ are of order $\Omega$ as in (5.30) and the WZ part is expanded as

$$
\begin{aligned}
T h_{5} & =T_{\mathrm{NR}} \Omega^{-2} h_{5}^{d i v}+T_{\mathrm{NR}} h_{5}^{f i n}+O\left(\Omega^{4}\right), \\
h_{5}^{d i v} & =\frac{\sqrt{s}}{3 !} \mathbf{L}_{0}^{\bar{A}_{1}} \mathbf{L}_{0}^{\bar{A}_{2}} \mathbf{L}_{0}^{\bar{A}_{3}} \bar{L}_{+0} \Gamma_{\bar{A}_{1} \cdots \bar{A}_{3}} i \sigma_{2} L_{+0} \\
h_{5}^{f i n} & =h_{2}^{(5)}+h_{1}^{(3)} \mathcal{F}_{1}
\end{aligned}
$$

with

$$
\begin{aligned}
& h_{2}^{(5)}=\frac{\sqrt{s}}{3 !}\left[\mathbf{L}_{0}^{\bar{A}_{1}} \mathbf{L}_{0}^{\bar{A}_{2}} \mathbf{L}_{0}^{\bar{A}_{3}} \bar{L}_{-1} \Gamma_{\bar{A}_{1} \cdots \bar{A}_{3}} \sigma_{1} L_{-1}+3 \mathbf{L}_{0}^{\bar{A}_{1}} \mathbf{L}_{0}^{\bar{A}_{2}} \mathbf{L}_{2}^{\bar{A}_{3}} \bar{L}_{+0} \Gamma_{\bar{A}_{1} \cdots \bar{A}_{3}} \sigma_{1} L_{+0}\right. \\
& +2 \mathbf{L}_{0}^{\bar{A}_{1}} \mathbf{L}_{0}^{\bar{A}_{2}} \mathbf{L}_{0}^{\bar{A}_{3}} \bar{L}_{+0} \Gamma_{\bar{A}_{1} \cdots \bar{A}_{3}} \sigma_{1} L_{+2}+6 \mathbf{L}_{0}^{\bar{A}_{1}} \mathbf{L}_{0}^{\bar{A}_{2}} \mathbf{L}_{1}^{\underline{A}_{3}} \bar{L}_{+0} \Gamma_{\bar{A}_{1} \cdots \bar{A}_{2} \underline{A}_{3}} \sigma_{1} L_{-1} \\
& +6 \mathbf{L}_{0}^{\bar{A}_{1}} \mathbf{L}_{1}^{\underline{A}_{2}} \mathbf{L}_{1}^{\underline{A}_{3}} \bar{L}_{+0} \Gamma_{\bar{A}_{1} \underline{A}_{2} \underline{A}_{3}} \sigma_{1} L_{+0}
\end{aligned}
$$

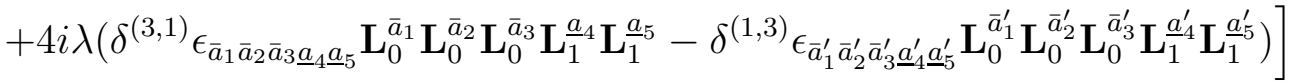

$$
\begin{aligned}
& h_{1}^{(3)}=\sqrt{s}\left[2 \mathbf{L}_{0}^{\bar{A}} \bar{L}_{+0} \Gamma_{\bar{A}} \varrho L_{-1}+\mathbf{L}_{1}^{\underline{A}} \bar{L}_{+0} \Gamma_{\underline{A}} \varrho L_{+0}\right] \text {, }
\end{aligned}
$$

where $\delta^{(m, n)}=1$ for a $(m, n)$-brane and $\delta^{(m, n)}=0$ for others. This implies that the bosonic 4 -form $C^{(4)}$ is expanded as

$$
\begin{aligned}
T d C^{(4)} & =T_{\mathrm{NR}} \Omega^{-2} d C_{0}^{(4)}+T_{\mathrm{NR}} d C_{2}^{(4)}+O\left(\Omega^{4}\right) \\
d C_{0}^{(4)} & =0 \\
d C_{2}^{(4)} & =\frac{\sqrt{s}}{3 !} 4 i \lambda\left(\delta^{(3,1)} \epsilon_{\bar{a}_{1} \bar{a}_{2} \bar{a}_{3} \underline{a}_{4} \underline{a}_{5}} e_{0}^{\bar{a}_{1}} e_{0}^{\bar{a}_{2}} e_{0}^{\bar{a}_{3}} e_{1}^{\underline{a}_{4}} e_{1}^{\underline{a}_{5}}-\delta^{(1,3)} \epsilon_{\bar{a}_{1}^{\prime} \bar{a}_{2}^{\prime} \bar{a}_{3}^{\prime} \underline{a}_{4}^{\prime} \underline{a}_{5}^{\prime}} e_{0}^{\bar{a}_{1}^{\prime}} e_{0}^{\bar{a}_{2}^{\prime}} e_{0}^{\bar{a}_{3}^{\prime}} e_{1}^{\frac{a_{4}^{\prime}}{e_{1}}} e_{1}^{\prime}\right)
\end{aligned}
$$

On the other hand, as $\mathcal{F}$ is of order $\Omega$, the DBI part is expanded as in (5.33). As was done in the $p=1$ case, we find

$$
d\left(\sqrt{s \operatorname{det} g_{0}} d^{4} \xi\right)=d\left(\operatorname{det}\left(\left(\mathbf{L}_{0}^{\bar{A}}\right)_{i}\right) d^{4} \xi\right)=-\frac{\sqrt{s}}{3 !} \mathbf{L}_{0}^{\bar{A}_{1}} \cdots \mathbf{L}_{0}^{\bar{A}_{3}} \bar{L}_{+0} \Gamma_{\bar{A}_{1} \cdots \bar{A}_{3}} i \sigma_{2} L_{+0}
$$

Thus the fermionic part contained in $\mathcal{L}_{\mathrm{WZ}}^{\text {div }}$ and $\mathcal{L}_{\mathrm{DBI}}^{\text {div }}$ cancel each other. In addition, the bosonic terms deleted by choosing

$$
C_{0}^{(4)}=-\frac{1}{4 !} \epsilon_{\bar{A}_{0} \cdots \bar{A}_{3}} e_{0}^{\bar{A}_{0}} \cdots e_{0}^{\bar{A}_{3}}
$$

which satisfies $d C_{0}^{(4)}=0$. Thus the matrix $M$ leads to the consistent non-relativistic limit of the AdS D3-brane.

The non-relativistic D3-brane action is given as (5.9) with (5.35) and

$$
\mathcal{L}_{\mathrm{WZ}}^{\mathrm{D} 3}=\int_{0}^{1} d t\left[\mathcal{C}_{2}^{(4)}+\mathcal{C}_{1}^{(2)} \hat{\mathcal{F}}_{1}\right]+C_{2}^{(4)}
$$


with

$$
\begin{aligned}
\mathcal{C}_{2}^{(4)}= & 2 c\left[\frac { 1 } { 3 ! } \hat { \mathbf { L } } _ { 0 } ^ { \overline { A } _ { 1 } } \hat { \mathbf { L } } _ { 0 } ^ { \overline { A } _ { 2 } } \hat { \mathbf { L } } _ { 0 } ^ { \overline { A } _ { 3 } } \left(\hat{\bar{L}}_{-1} \Gamma_{\bar{A}_{1} \bar{A}_{2} \bar{A}_{3}} i \sigma_{2} \theta_{-}+\hat{\bar{L}}_{+2} \Gamma_{\left.\bar{A}_{1} \bar{A}_{2} \bar{A}_{3} i \sigma_{2} \theta_{+}\right)}\right.\right. \\
& +\frac{1}{2} \hat{\mathbf{L}}_{0}^{\bar{A}_{1}} \hat{\mathbf{L}}_{0}^{\bar{A}_{2}} \hat{\mathbf{L}}_{1}^{\underline{A}_{3}}\left(\hat{\bar{L}}_{-1} \Gamma_{\bar{A}_{1} \bar{A}_{2} \underline{A}_{3}} i \sigma_{2} \theta_{+}+\hat{\bar{L}}_{+0} \Gamma_{\bar{A}_{1} \bar{A}_{2} \underline{A}_{3}} i \sigma_{2} \theta_{-}\right) \\
& \left.+\hat{\mathbf{L}}_{0}^{\bar{A}_{1}} \hat{\mathbf{L}}_{0}^{\bar{A}_{2}} \hat{\mathbf{L}}_{2}^{\bar{A}_{3}} \hat{\bar{L}}_{+0} \Gamma_{\bar{A}_{1} \bar{A}_{2} \bar{A}_{3}} i \sigma_{2} \theta_{+}+\hat{\mathbf{L}}_{0}^{\bar{A}_{1}} \hat{\mathbf{L}}_{1}^{\underline{A}_{2}} \hat{\mathbf{L}}_{1}^{\underline{A}_{3}} \hat{\bar{L}}_{+0} \Gamma_{\bar{A}_{1} \underline{A}_{2} \underline{A}_{3}} i \sigma_{2} \theta_{+}\right] \\
\mathcal{C}_{1}^{(2)}= & 2 c\left(\hat{\mathbf{L}}_{0}^{\bar{A}}\left(\hat{\bar{L}}_{+0} \Gamma_{\bar{A}} \varrho \theta_{-}+\hat{\bar{L}}_{-1} \Gamma_{\bar{A}} \varrho \theta_{+}\right)+\hat{\mathbf{L}}_{1}^{A} \hat{\bar{L}}_{+0} \Gamma_{\underline{A}} \varrho \theta_{+}\right) .
\end{aligned}
$$

The bosonic contribution $C_{2}^{(4)}$ is

$$
\begin{aligned}
\int_{\Sigma} C_{2}^{(4)} & =4 i \sqrt{s} \lambda \int_{\Sigma}\left[\delta^{(3,1)} \operatorname{vol}_{\Sigma_{3}} \epsilon_{a \underline{b}} d y^{\underline{a}} y^{\underline{\underline{b}}}-\delta^{(1,3)} \operatorname{vol}_{\Sigma_{3}^{\prime}} \epsilon_{\underline{a}^{\prime} \underline{b}^{\prime}} d y^{\underline{a^{\prime}}} y^{\underline{b}^{\prime}}\right] \\
& =4 i \sqrt{s} \lambda \int d^{4} \xi \sqrt{s \operatorname{det} g_{0}}\left[\delta^{(3,1)} \epsilon_{\underline{a b}} \partial_{\xi^{\prime}} y^{\underline{a}} y^{\underline{\underline{b}}}-\delta^{(1,3)} \epsilon_{\underline{a}^{\prime} \underline{b}^{\prime}} \partial_{\xi} y^{\underline{a}^{\prime}} y^{\underline{b^{\prime}}}\right]
\end{aligned}
$$

where $\Sigma_{m} \times \Sigma_{n}^{\prime}$ is the $(m, n)$-brane worldvolume, and $\operatorname{vol}_{\Sigma_{\ell}}=\frac{1}{\ell !} \epsilon_{\bar{a}_{1} \cdots \bar{a}_{\ell}} e_{0}^{\bar{a}_{1}} \cdots e_{0}^{\bar{a}_{\ell}} . \quad \xi\left(\xi^{\prime}\right)$ represents the worldvolume direction in $\operatorname{AdS}_{5}\left(\mathrm{~S}^{5}\right.$ respectively). By fixing the $\kappa$-symmetry as $\theta_{+}=0$, the non-relativistic action is simplified as

$$
\begin{aligned}
S_{\mathrm{NR}}^{D 3}= & T_{\mathrm{NR}} \int d^{4} \xi \sqrt{s \operatorname{det} g_{0}}\left[\frac{1}{2} g_{0}^{i j} \partial_{i} y^{\underline{A}} \partial_{j} y^{\underline{B}} \eta_{\underline{A B}}+\frac{\lambda^{2}}{2}\left(m y^{2}-n y^{\prime 2}\right)\right. \\
& \left.+2 i \bar{\theta}_{-} \gamma^{i} \mathrm{D}_{j} \theta_{-}+\frac{1}{4}\left(F_{1}\right)_{i j}\left(F_{1}\right)^{i j}\right]+T_{\mathrm{NR}} \int_{\Sigma} C_{2}^{(4)}
\end{aligned}
$$

\subsection{D5-brane}

Fourthly, we consider a D5-brane for which $\varrho=\sigma_{1}$ and $\sigma=\sigma_{3}$. The gluing matrix is

$$
M=\sqrt{-s} \Gamma^{\bar{A}_{0} \cdots \bar{A}_{5}} \otimes \rho, \quad \rho=\sigma_{1}, \sigma_{3}, 1 .
$$

Since

$$
\begin{aligned}
& M^{\prime} \Gamma_{\bar{B}_{1} \cdots \bar{B}_{5}} \varrho= \pm \Gamma_{\bar{B}_{1} \cdots \bar{B}_{5}} \varrho M, \quad M^{\prime} \Gamma_{\bar{A}} \varrho= \pm \Gamma_{\bar{A}} \varrho M, \quad \rho=\left\{\begin{array}{l}
\sigma_{1}, 1 \\
\sigma_{3}
\end{array}\right. \\
& M^{\prime} \Gamma_{\bar{B}_{1} \cdots \bar{B}_{3}} i \sigma_{2}= \pm \Gamma_{\bar{B}_{1} \cdots \bar{B}_{5}} i \sigma_{2} M, \quad \rho=\left\{\begin{array}{l}
1 \\
\sigma_{1}, \sigma_{3}
\end{array}\right. \\
& M^{\prime} \Gamma_{\bar{A}} \sigma= \pm \Gamma_{\bar{A}} \sigma M, \quad \rho=\left\{\begin{array}{l}
\sigma_{3}, 1 \\
\sigma_{1}
\end{array}\right.
\end{aligned}
$$


$\mathcal{F}$ is of order $\Omega$ only for $\rho=\sigma_{1}$. In this case the WZ part is expanded as

$$
\begin{aligned}
T h_{7} & =T_{\mathrm{NR}} \Omega^{-2} h_{7}^{d i v}+T_{\mathrm{NR}} h_{7}^{f i n}+O\left(\Omega^{4}\right), \\
h_{7}^{d i v} & =\frac{\sqrt{s}}{5 !} \mathbf{L}_{0}^{\bar{A}_{1}} \cdots \mathbf{L}_{0}^{\bar{A}_{5}} \bar{L}_{+0} \Gamma_{\bar{A}_{1} \cdots \bar{A}_{5}} \varrho L_{+0} \\
h_{7}^{f i n} & =h_{2}^{(7)}+h_{1}^{(5)} \mathcal{F}_{1}+\frac{1}{2} h_{0}^{(3)} \mathcal{F}_{1}^{2}
\end{aligned}
$$

and the DBI part is expanded as (5.33). We find that $h_{2}^{(7)}, h_{1}^{(5)}$ and $h_{0}^{(3)}$ are given as

$$
\begin{aligned}
& h_{2}^{(7)}=\frac{\sqrt{s}}{5 !}\left[\mathbf{L}_{0}^{\bar{A}_{1}} \cdots \mathbf{L}_{0}^{\bar{A}_{5}} \bar{L}_{-1} \Gamma_{\bar{A}_{1} \cdots \bar{A}_{5}} \varrho L_{-1}+5 \mathbf{L}_{0}^{\bar{A}_{1}} \cdots \mathbf{L}_{0}^{\bar{A}_{4}} \mathbf{L}_{2}^{\bar{A}_{5}} \bar{L}_{+0} \Gamma_{\bar{A}_{1} \cdots \bar{A}_{5}} \varrho L_{+0}\right.
\end{aligned}
$$

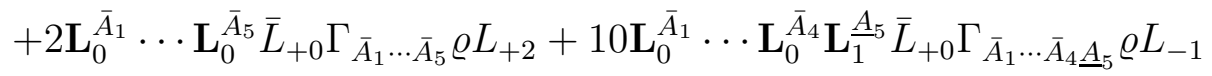

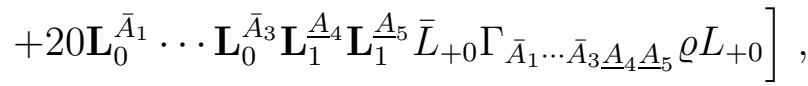

$$
\begin{aligned}
& h_{1}^{(5)}=\frac{\sqrt{s}}{3 !}\left[2 \mathbf{L}_{0}^{\bar{A}_{1}} \mathbf{L}_{0}^{\bar{A}_{2}} \mathbf{L}_{0}^{\bar{A}_{3}} \bar{L}_{+0} \Gamma_{\bar{A}_{1} \bar{A}_{2} \bar{A}_{3}} i \sigma_{2} L_{-1}+3 \mathbf{L}_{0}^{\bar{A}_{1}} \mathbf{L}_{0}^{\bar{A}_{2}} \mathbf{L}_{1}^{\underline{A}_{3}} \bar{L}_{+0} \Gamma_{\bar{A}_{1} \bar{A}_{2} \underline{A}_{3}} i \sigma_{2} L_{+0}\right.
\end{aligned}
$$

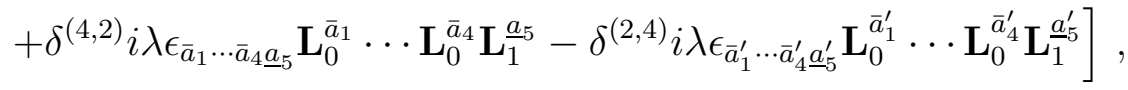

$$
\begin{aligned}
& h_{0}^{(3)}=\sqrt{s} \mathbf{L}_{0}^{\bar{A}} \bar{L}_{+0} \Gamma_{\bar{A}} \varrho L_{+0} \text {. }
\end{aligned}
$$

This implies that the bosonic 6 -form $C^{(6)}$ is expanded as

$$
\begin{aligned}
T d C^{(6)} & =T_{\mathrm{NR}} \Omega^{-2} d C_{0}^{(6)}+T_{\mathrm{NR}} d C_{2}^{(6)}+O\left(\Omega^{4}\right) \\
d C_{0}^{(6)} & =0 \\
d C_{2}^{(6)} & =\frac{i \sqrt{s}}{3 !} \lambda\left[\delta^{(4,2)} \epsilon_{\bar{a}_{1} \cdots \bar{a}_{4} \underline{a}_{5}} e_{0}^{\bar{a}_{1}} \cdots e_{0}^{\bar{a}_{4}} e_{1}^{\underline{a}_{5}}-\delta^{(2,4)} \epsilon_{\bar{a}_{1}^{\prime} \cdots \bar{a}_{4}^{\prime} \underline{a}_{5}^{\prime}} e_{0}^{\bar{a}_{1}^{\prime}} \cdots e_{0}^{\bar{a}_{4}^{\prime}} e_{1}^{\underline{a}_{5}^{\prime}}\right] F_{1} .
\end{aligned}
$$

Because

$$
d\left(\sqrt{s \operatorname{det} g_{0}} d^{6} \xi\right)=-i \frac{\sqrt{s}}{5 !} \mathbf{L}_{0}^{\bar{A}_{1}} \cdots \mathbf{L}_{0}^{\bar{A}_{5}} \bar{L}_{+0} \Gamma_{\bar{A}_{1} \cdots \bar{A}_{5}} \varrho L_{+0}
$$

$h_{7}^{d i v}$ in (15.59) and the fermionic term in the DBI part $\mathcal{L}_{\mathrm{DBI}}^{\text {div }}$ cancel each other. As before, one sees that the bosonic terms are also deleted by choosing $C_{0}^{(6)}=-\frac{1}{6 !} \epsilon_{\bar{A}_{0} \cdots \bar{A}_{5}} e_{0}^{\bar{A}_{0}} \cdots e_{0}^{\bar{A}_{5}}$. Summarizing we have shown that the matrix $M$ with $\varrho=\sigma_{1}$ leads to the consistent non-relativistic limit of the AdS D5-brane ${ }^{5}$.

The non-relativistic D5-brane action is given as (5.9) with (5.35) and

$$
\mathcal{L}_{\mathrm{WZ}}^{\mathrm{D} 5}=\int_{0}^{1} d t\left[\mathcal{C}_{2}^{(6)}+\mathcal{C}_{1}^{(4)} \hat{\mathcal{F}}_{1}+\frac{1}{2} \mathcal{C}_{0}^{(2)} \hat{\mathcal{F}}_{1}^{2}\right]+C_{2}^{(6)}
$$

\footnotetext{
${ }^{5}$ It is now obvious that for NS5-brane with $\varrho=\sigma_{3}$ and $\sigma=-\sigma_{1}$ the gluing matrix (5.54) with $\rho=\sigma_{3}$ leads to the consistent non-relativistic NS5-brane.
} 
with

$$
\begin{aligned}
& \mathcal{C}_{2}^{(6)}=2 c\left[\frac{1}{5 !} \hat{\mathbf{L}}_{0}^{\bar{A}_{1}} \cdots \hat{\mathbf{L}}_{0}^{\bar{A}_{5}}\left(\hat{\bar{L}}_{-1} \Gamma_{\bar{A}_{1} \cdots \bar{A}_{5}} \varrho \theta_{-}+\hat{\bar{L}}_{+2} \Gamma_{\bar{A}_{1} \cdots \bar{A}_{5}} \varrho \theta_{+}\right)\right. \\
& +\frac{1}{4 !} \hat{\mathbf{L}}_{0}^{\bar{A}_{1}} \cdots \hat{\mathbf{L}}_{0}^{\bar{A}_{4}} \hat{\mathbf{L}}_{1}^{\underline{A}_{5}}\left(\hat{\bar{L}}_{-1} \Gamma_{\bar{A}_{1} \cdots \bar{A}_{4} \underline{A}_{5}} \varrho \theta_{+}+\hat{\bar{L}}_{+0} \Gamma_{\bar{A}_{1} \cdots \bar{A}_{4} \underline{A}_{5}} \varrho \theta_{-}\right) \\
& +\frac{1}{4 !} \hat{\mathbf{L}}_{0}^{\bar{A}_{1}} \cdots \hat{\mathbf{L}}_{0}^{\bar{A}_{4}} \hat{\mathbf{L}}_{2}^{\bar{A}_{5}} \hat{\bar{L}}_{+0} \Gamma_{\bar{A}_{1} \cdots \bar{A}_{5}} \varrho \theta_{+}
\end{aligned}
$$

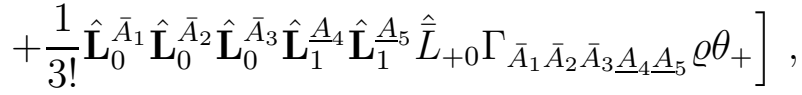

$$
\begin{aligned}
& \mathcal{C}_{1}^{(4)}=2 c\left[\frac{1}{3 !} \hat{\mathbf{L}}_{0}^{\bar{A}_{1}} \hat{\mathbf{L}}_{0}^{\bar{A}_{2}} \hat{\mathbf{L}}_{0}^{\bar{A}_{3}}\left(\hat{\bar{L}}_{+0} \Gamma_{\bar{A}_{1} \bar{A}_{2} \bar{A}_{3}} i \sigma_{2} \theta_{-}+\hat{\bar{L}}_{-1} \Gamma_{\bar{A}_{1} \bar{A}_{2} \bar{A}_{3}} i \sigma_{2} \theta_{+}\right)\right. \\
& \left.+\frac{1}{2} \hat{\mathbf{L}}_{0}^{\bar{A}_{1}} \hat{\mathbf{L}}_{0}^{\bar{A}_{2}} \hat{\mathbf{L}}_{1}^{\underline{A}_{3}} \hat{\bar{L}}_{+0} \Gamma_{\bar{A}_{1} \bar{A}_{2} \underline{A}_{3}} i \sigma_{2} \theta_{+}\right] \\
& \mathcal{C}_{0}^{(2)}=2 c\left[\hat{\mathbf{L}}_{0}^{\bar{A}} \hat{\bar{L}}_{+0} \Gamma_{\bar{A}} \varrho \theta_{+}\right] \text {. }
\end{aligned}
$$

The bosonic contribution is

$$
\begin{aligned}
\int_{\Sigma} C_{2}^{(6)} & =4 i \sqrt{s} \lambda \int_{\Sigma}\left[\delta^{(4,2)} \operatorname{vol}_{\Sigma_{4}} y F_{1}-\delta^{(2,4)} \operatorname{vol}_{\Sigma_{4}^{\prime}} y^{\prime} F_{1}\right] \\
& =-4 i \sqrt{s} \lambda \int d^{6} \xi \sqrt{s \operatorname{det} g_{0}}\left[\delta^{(4,2)} \partial_{i^{\prime}} y\left({ }^{*} A_{1}\right)^{i^{\prime}}-\delta^{(2,4)} \partial_{i} y\left({ }^{*} A_{1}\right)^{i}\right]
\end{aligned}
$$

where $y\left(y^{\prime}\right)$ is the transverse direction in $\operatorname{AdS}_{5}\left(\mathrm{~S}^{5}\right)$, and $i\left(i^{\prime}\right)$ represents the worldvolume directions in $\operatorname{AdS}_{5}\left(\mathrm{~S}^{5}\right)$. * means the Hodge dual in $\Sigma_{2}$ or $\Sigma_{2}^{\prime}$. The $\kappa$-gauge symmetry is fixed by $\theta_{+}=0$, and the non-relativistic action is simplified as

$$
\begin{aligned}
S_{\mathrm{NR}}^{D 5}= & T_{\mathrm{NR}} \int d^{6} \xi \sqrt{s \operatorname{det} g_{0}}\left[\frac{1}{2} g_{0}^{i j} \partial_{i} y^{\underline{A}} \partial_{j} y_{\underline{A}}+\frac{\lambda^{2}}{2}\left(m y^{2}-n y^{\prime 2}\right)\right. \\
& \left.+2 i \bar{\theta}_{-} \gamma^{i} \mathrm{D}_{i} \theta_{-}+\frac{1}{4}\left(F_{1}\right)_{i j}\left(F_{1}\right)^{i j}\right]+T_{\mathrm{NR}} \int_{\Sigma} C_{2}^{(6)} .
\end{aligned}
$$

\subsection{D7-brane}

Finally, let us consider a D7-brane for which $\varrho=\sigma_{1}$ and $\sigma=\sigma_{3}$. By using the gluing matrix

$$
M=\sqrt{-s} \Gamma^{\bar{A}_{0} \cdots \bar{A}_{6}} \otimes i \sigma_{2}
$$

one derives

$$
\begin{array}{ll}
M^{\prime} \Gamma_{\bar{B}_{1} \cdots \bar{B}_{7}} i \sigma_{2}=\Gamma_{\bar{B}_{1} \cdots \bar{B}_{7}} i \sigma_{2} M, & M^{\prime} \Gamma_{\bar{B}_{1} \cdots \bar{B}_{5}} \varrho=-\Gamma_{\bar{B}_{1} \cdots \bar{B}_{5}} \varrho M \\
M^{\prime} \Gamma_{\bar{B}_{1} \cdots \bar{B}_{3}} i \sigma_{2}=\Gamma_{\bar{B}_{1} \cdots \bar{B}_{3}} i \sigma_{2} M, & M^{\prime} \Gamma_{\bar{A}} \varrho=-\Gamma_{\bar{A}} \varrho M \\
M^{\prime} \Gamma_{\bar{A}} \sigma=-\Gamma_{\bar{A}} \sigma M .
\end{array}
$$


These imply that $\mathcal{F}$ is of order $\Omega$ and the $\mathrm{WZ}$ part is expanded as

$$
\begin{aligned}
T h_{9} & =T_{\mathrm{NR}} \Omega^{-2} h_{9}^{d i v}+T_{\mathrm{NR}} h_{9}^{f i n}+O\left(\Omega^{4}\right), \\
h_{9}^{d i v} & =\frac{\sqrt{s}}{7 !} \mathbf{L}_{0}^{\bar{A}_{1}} \cdots \mathbf{L}_{0}^{\bar{A}_{7}} \bar{L}_{+0} \Gamma_{\bar{A}_{1} \cdots \bar{A}_{7}} i \sigma_{2} L_{+0}, \\
h_{9}^{f i n} & =h_{2}^{(9)}+h_{1}^{(7)} \mathcal{F}_{1}+\frac{1}{2} h_{0}^{(5)} \mathcal{F}_{1}^{2}
\end{aligned}
$$

and the DBI is as in (5.33) $)$. It is straightforward to see that $h_{2}^{(9)}, h_{1}^{(7)}$ and $h_{0}^{(5)}$ are given as

$$
\begin{aligned}
& h_{2}^{(9)}=\frac{\sqrt{s}}{7 !}\left[\mathbf{L}_{0}^{\bar{A}_{1}} \cdots \mathbf{L}_{0}^{\bar{A}_{7}} \bar{L}_{-1} \Gamma_{\bar{A}_{1} \cdots \bar{A}_{7}} i \sigma_{2} L_{-1}+7 \mathbf{L}_{0}^{\bar{A}_{1}} \cdots \mathbf{L}_{0}^{\bar{A}_{6}} \mathbf{L}_{2}^{\bar{A}_{7}} \bar{L}_{+0} \Gamma_{\bar{A}_{1} \cdots \bar{A}_{7}} i \sigma_{2} L_{+0}\right. \\
& +2 \mathbf{L}_{0}^{\bar{A}_{1}} \cdots \mathbf{L}_{0}^{\bar{A}_{7}} \bar{L}_{+0} \Gamma_{\bar{A}_{1} \cdots \bar{A}_{7}} i \sigma_{2} L_{+2}+14 \mathbf{L}_{0}^{\bar{A}_{1}} \cdots \mathbf{L}_{0}^{\bar{A}_{6}} \mathbf{L}_{1}^{\underline{A}_{7}} \bar{L}_{+0} \Gamma_{\bar{A}_{1} \cdots \bar{A}_{6} \underline{A}_{7}} i \sigma_{2} L_{-1}
\end{aligned}
$$

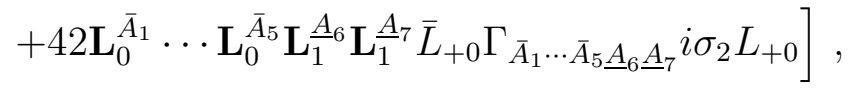

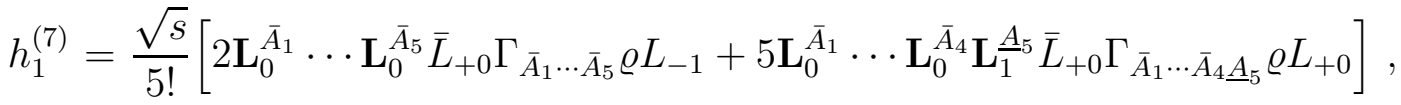

$$
\begin{aligned}
& h_{0}^{(5)}=\frac{\sqrt{s}}{3 !}\left[\mathbf{L}_{0}^{\bar{A}_{1}} \cdots \mathbf{L}_{0}^{\bar{A}_{3}} \bar{L}_{+0} \Gamma_{\bar{A}_{1} \cdots \bar{A}_{3}} i \sigma_{2} L_{+0}\right.
\end{aligned}
$$

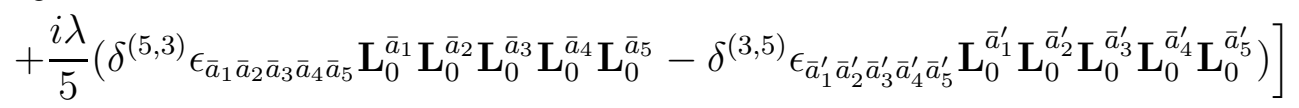

This implies that the bosonic 8-form $C^{(8)}$ is expanded as

$$
\begin{aligned}
T d C^{(8)} & =T_{\mathrm{NR}} \Omega^{-2} d C_{0}^{(8)}+T_{\mathrm{NR}} d C_{2}^{(8)}+O\left(\Omega^{4}\right) \\
d C_{0}^{(8)} & =0 \\
d C_{2}^{(8)} & =\frac{2 i \sqrt{s}}{5 !} \lambda\left[\delta^{(5,3)} \epsilon_{\bar{a}_{1} \cdots \bar{a}_{5}} e_{0}^{\bar{a}_{1}} \cdots e_{0}^{\bar{a}_{5}}-\delta^{(3,5)} \epsilon_{\bar{a}_{1}^{\prime} \cdots \bar{a}_{5}^{\prime}} e_{0}^{\bar{a}_{1}^{\prime}} \cdots e_{0}^{\bar{a}_{5}^{\prime}}\right]\left(F_{1}\right)^{2} .
\end{aligned}
$$

As before, we find

$$
d\left(\sqrt{s \operatorname{det} g_{0}} d^{8} \xi\right)=d\left(\operatorname{det}\left(\left(\mathbf{L}_{0}^{\bar{A}}\right)_{i}\right) d^{8} \xi\right)=-i \frac{\sqrt{s}}{7 !} \mathbf{L}_{0}^{\bar{A}_{1}} \cdots \mathbf{L}_{0}^{\bar{A}_{7}} \bar{L}_{+0} \Gamma_{\bar{A}_{1} \cdots \bar{A}_{7}} i \sigma_{2} L_{+0} .
$$

This implies that $h_{9}^{d i v}$ and the fermionic terms in $\mathcal{L}_{\mathrm{DBI}}^{\text {div }}$ cancel each other. The bosonic terms are also deleted by choosing $C_{0}^{(8)}=-\frac{1}{8 !} \epsilon_{\bar{A}_{0} \cdots \bar{A}_{7}} e_{0}^{\bar{A}_{0}} \cdots e_{0}^{\bar{A}_{7}}$. Thus we find that the matrix $M$ leads to the consistent non-relativistic limit of the AdS D7-brane.

The non-relativistic D7-brane action is given as (5.9) with (5.35) and

$$
\mathcal{L}_{\mathrm{WZ}}^{\mathrm{D} 7}=\int_{0}^{1} d t\left[\mathcal{C}_{2}^{(8)}+\mathcal{C}_{1}^{(6)} \hat{\mathcal{F}}_{1}+\frac{1}{2} \mathcal{C}_{0}^{(4)} \hat{\mathcal{F}}_{1}^{2}\right]+C_{2}^{(8)}
$$


with

$$
\begin{aligned}
& \mathcal{C}_{2}^{(8)}=2 c\left[\frac{1}{7 !} \hat{\mathbf{L}}_{0}^{\bar{A}_{1}} \cdots \hat{\mathbf{L}}_{0}^{\bar{A}_{7}}\left(\hat{\bar{L}}_{-1} \Gamma_{\bar{A}_{1} \cdots \bar{A}_{7}} i \sigma_{2} \theta_{-}+\hat{\bar{L}}_{+2} \Gamma_{\bar{A}_{1} \cdots \bar{A}_{7}} i \sigma_{2} \theta_{+}\right)\right. \\
& +\frac{1}{6 !} \hat{\mathbf{L}}_{0}^{\bar{A}_{1}} \cdots \hat{\mathbf{L}}_{0}^{\bar{A}_{6}} \hat{\mathbf{L}}_{1}^{\underline{A}_{7}}\left(\hat{\bar{L}}_{-1} \Gamma_{\bar{A}_{1} \cdots \bar{A}_{6} \underline{A}_{7}} i \sigma_{2} \theta_{+}+\hat{\bar{L}}_{+0} \Gamma_{\bar{A}_{1} \cdots \bar{A}_{6} \underline{A}_{7}} i \sigma_{2} \theta_{-}\right) \\
& +\frac{1}{6 !} \hat{\mathbf{L}}_{0}^{\bar{A}_{1}} \cdots \hat{\mathbf{L}}_{0}^{\bar{A}_{6}} \hat{\mathbf{L}}_{2}^{\bar{A}_{7}} \hat{\bar{L}}_{+0} \Gamma_{\bar{A}_{1} \cdots \bar{A}_{7}} i \sigma_{2} \theta_{+}
\end{aligned}
$$

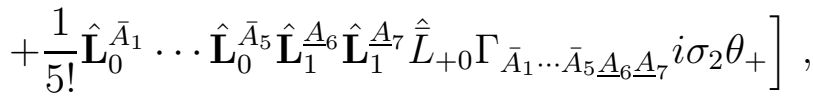

$$
\begin{aligned}
& \mathcal{C}_{1}^{(6)}=2 c\left[\frac{1}{5 !} \hat{\mathbf{L}}_{0}^{\bar{A}_{1}} \cdots \hat{\mathbf{L}}_{0}^{\bar{A}_{5}}\left(\hat{\bar{L}}_{+0} \Gamma_{\bar{A}_{1} \cdots \bar{A}_{5}} \varrho \theta_{-}+\hat{\bar{L}}_{-1} \Gamma_{\bar{A}_{1} \cdots \bar{A}_{5}} \varrho \theta_{+}\right)\right.
\end{aligned}
$$

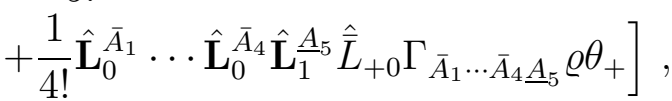

$$
\begin{aligned}
& \mathcal{C}_{0}^{(4)}=2 c\left[\frac{1}{3 !} \hat{\mathbf{L}}_{0}^{\bar{A}_{1}} \cdots \hat{\mathbf{L}}_{0}^{\bar{A}_{3}} \hat{\bar{L}}_{+0} \Gamma_{\bar{A}_{1} \cdots \bar{A}_{3}} i \sigma_{2} \theta_{+}\right] .
\end{aligned}
$$

The bosonic contribution is

$$
\begin{aligned}
\int_{\Sigma} C_{2}^{(8)} & =-2 i \sqrt{s} \lambda \int_{\Sigma}\left[\delta^{(5,3)} \operatorname{vol}_{\Sigma_{5}} A_{1} F_{1}-\delta^{(3,5)} \operatorname{vol}_{\Sigma_{5}^{\prime}} A_{1} F_{1}\right] \\
& =-2 i \sqrt{s} \lambda \int d^{8} \xi \sqrt{s \operatorname{det} g_{0}}\left[\delta^{(5,3)}\left(A_{1}\right)_{i^{\prime}}\left({ }^{*} F_{1}\right)^{i^{\prime}}-\delta^{(3,5)}\left(A_{1}\right)_{i}\left({ }^{*} F_{1}\right)^{i}\right]
\end{aligned}
$$

where $i\left(i^{\prime}\right)$ represents worldvolume directions in $\mathrm{AdS}_{5}\left(\mathrm{~S}^{5}\right)$, and $*$ means the Hodge dual in $\Sigma_{3}$ or $\Sigma_{3}^{\prime}$. The $\kappa$-gauge symmetry is fixed by $\theta_{+}=0$, and then the non-relativistic action is simplified as

$$
\begin{aligned}
S_{\mathrm{NR}}^{D 7}= & T_{\mathrm{NR}} \int d^{8} \xi \sqrt{s \operatorname{det} g_{0}}\left[\frac{1}{2} g_{0}^{i j} \partial_{i} y^{\underline{A}} \partial_{j} y_{\underline{A}}+\frac{\lambda^{2}}{2}\left(m y^{2}-n y^{\prime 2}\right)\right. \\
& \left.+2 i \bar{\theta}_{-} \gamma^{i} \mathrm{D}_{i} \theta_{-}+\frac{1}{4}\left(F_{1}\right)_{i j}\left(F_{1}\right)^{i j}\right]+T_{\mathrm{NR}} \int_{\Sigma} C_{2}^{(8)} .
\end{aligned}
$$

In summary, we have derived non-relativistic $\mathrm{AdS} D p$-brane actions in $\operatorname{AdS}_{5} \times \mathrm{S}^{5}$. In the flat limit $\lambda \rightarrow 0$, these actions for Lorentzian branes are reduced to the non-relativistic $\mathrm{D} p$-brane actions in flat spacetime derived in [12].

\section{NH Superalgebra of Branes in $\mathrm{AdS}_{4 / 7} \times \mathrm{S}^{7 / 4}$}

The super-isometry algebra of the $\operatorname{AdS}_{q+2} \times \mathrm{S}^{9-q}(q=2,5)$ solution of the eleven-dimensional supergravity is generated by translation $P_{A}$, Lorentz rotation $J_{A B}=\left(J_{a b}, J_{a^{\prime} b^{\prime}}\right)$ and 32- 
component Majorana supercharge $Q$ as

$$
\begin{aligned}
& {\left[P_{a}, P_{b}\right]=4 \epsilon^{2} \lambda^{2} J_{a b}, \quad\left[P_{a^{\prime}}, P_{b^{\prime}}\right]=-\epsilon^{2} \lambda^{2} J_{a^{\prime} b^{\prime}},} \\
& {\left[J_{a b}, P_{c}\right]=\eta_{b c} P_{a}-\eta_{a c} P_{b}, \quad\left[J_{a^{\prime} b}, P_{c^{\prime}}\right]=\eta_{b^{\prime} c^{\prime}} P_{a^{\prime}}-\eta_{a^{\prime} c^{\prime}} P_{b^{\prime}},} \\
& {\left[J_{a b}, J_{c d}\right]=\eta_{b c} J_{a d}+3 \text {-terms }, \quad\left[J_{a^{\prime} b^{\prime}}, J_{c^{\prime} d^{\prime}}\right]=\eta_{b^{\prime} c^{\prime}} J_{a^{\prime} d^{\prime}}+3 \text {-terms }} \\
& {\left[P_{A}, Q\right]=-\frac{\lambda}{2} Q \widehat{\Gamma}_{A}, \quad\left[J_{A B}, Q\right]=\frac{1}{2} Q \Gamma_{A B},} \\
& \{Q, Q\}=-2 \mathcal{C} \Gamma^{A} P_{A}+\lambda \mathcal{C} \widehat{\Gamma}^{A B} J_{A B}
\end{aligned}
$$

where $\widehat{\Gamma}^{A}=\left(2 \mathcal{I} \Gamma^{a}, \mathcal{I} \Gamma^{a^{\prime}}\right)$ and $\widehat{\Gamma}^{A B}=\left(2 \mathcal{I} \Gamma^{a b},-\mathcal{I} \Gamma^{a^{\prime} b^{\prime}}\right)$, and $\epsilon^{2}=1$ for $q=2$ and $\epsilon^{2}=-1$ for $q=5$. For $q=2$, this superalgebra is the super- $\mathrm{AdS}_{4} \times \mathrm{S}^{7}$ algebra, $\operatorname{osp}(8 \mid 4)$, with the vector index of $\mathrm{AdS}_{4}, a=0,1,2,3$ and that of $\mathrm{S}^{7}, a^{\prime}=4,5, \ldots, 9, \downarrow$. On the other hand, for $q=5$, this superalgebra is the super- $\mathrm{AdS}_{7} \times \mathrm{S}^{4}$ algebra, $\operatorname{osp}\left(8^{*} \mid 4\right)$ with the vector index of $\mathrm{S}^{4}, a=\natural, 1,2,3$ and that of $\mathrm{AdS}_{7}, a^{\prime}=4,5, \ldots, 9,0$. We use the almost positive metric $\eta_{\mu \nu}$. We define $\lambda$ and $\mathcal{I}$ as

$$
\begin{aligned}
& \lambda=\frac{1}{R}, \quad R^{2}=2 k R_{S}^{2}=\frac{1}{k} R_{A d S}^{2}, \quad k= \begin{cases}1 / 2, & q=2 \\
2, & q=5\end{cases} \\
& \mathcal{I}=\Gamma^{\sharp 123}, \quad \Gamma^{\sharp}= \begin{cases}\Gamma^{0} & q=2 \\
-\Gamma^{\natural} & q=5\end{cases}
\end{aligned}
$$

where $R_{S}$ and $R_{A d S}$ are the radii of $\mathrm{S}^{9-q}$ and that of $\mathrm{AdS}_{q+2}$, respectively. The gamma matrix $\Gamma^{A} \in \operatorname{Spin}(1,10)$ and the charge conjugation matrix $\mathcal{C}$ satisfy (2.2)

Letting $g$ be a group element of the supergroup of the superalgebra (6.1), the LI Cartan one-form is defined as

$$
\Omega=g^{-1} d g=\mathbf{L}^{A} P_{A}+\frac{1}{2} \mathbf{L}^{A B} J_{A B}+Q_{\alpha} L^{\alpha} .
$$

The commutation relations $\left[T_{\hat{A}}, T_{\hat{B}}\right\}=f_{\hat{A} \hat{B}} \hat{C} T_{\hat{C}}, T_{\hat{A}}=\left\{P_{A}, J_{A B}, Q_{I}\right\}$, are equivalent to the Maurer-Cartan (MC) equation

$$
d \Omega=-\Omega \wedge \Omega
$$

The MC equations corresponding to the superalgebra (6.1) are derived as

$$
\begin{aligned}
d \mathbf{L}^{A} & =-\eta_{B C} \mathbf{L}^{A B} \mathbf{L}^{C}-\bar{L} \Gamma^{A} L \\
d \mathbf{L}^{a b} & =-4 \epsilon^{2} \lambda^{2} \mathbf{L}^{a} \mathbf{L}^{b}-\eta_{c d} \mathbf{L}^{c a} \mathbf{L}^{b d}+2 \lambda \bar{L} \mathcal{I} \Gamma^{a b} L, \\
d \mathbf{L}^{a^{\prime} b^{\prime}} & =+\epsilon^{2} \lambda^{2} \mathbf{L}^{a^{\prime}} \mathbf{L}^{b^{\prime}}-\eta_{c^{\prime} d^{\prime}} \mathbf{L}^{c^{\prime} a^{\prime}} \mathbf{L}^{b^{\prime} d^{\prime}}-\lambda \bar{L} \mathcal{I} \Gamma^{a^{\prime} b^{\prime}} L, \\
d L^{\alpha} & =\frac{\lambda}{2} \mathbf{L}^{A} \widehat{\Gamma}_{A} L-\frac{1}{4} \mathbf{L}^{A B} \Gamma_{A B} L .
\end{aligned}
$$




\begin{tabular}{|c|c|c|c|c|c|}
\hline 1-brane & 2-brane & 5-brane & 6-brane & 9-brane & 10-brane \\
\hline$(1,1)$ & $(0,3),(2,1)$ & $(1,5),(3,3)$ & $(0,7),(2,5),(4,3)$ & $(3,7)$ & $(4,7)$ \\
\hline
\end{tabular}

Table 4: Branes in $\mathrm{AdS}_{4 / 7} \times \mathrm{S}^{7 / 4}$

We introduce a matrix $M$

$$
M=\ell \Gamma^{\bar{A}_{0} \cdots \bar{A}_{p}}, \quad \ell^{2}(-1)^{\left[\frac{p+1}{2}\right]} s=1
$$

where $\left\{\bar{A}_{0}, \cdots, \bar{A}_{p}\right\}$ are directions along which the brane worldvolume extends, so $A=$ $(\bar{A}, \underline{A})$. Let an AdS brane extend along $m$ directions in $\mathrm{AdS}_{4}$ or $\mathrm{S}^{4}$ and $n$ directions in $\mathrm{S}^{7}$ or $\mathrm{AdS}_{7}$, then the AdS brane worldvolume admits $\operatorname{AdS}_{m}\left(\mathrm{H}^{m}\right) \times \mathrm{S}^{n}$ or $\mathrm{S}^{m} \times \mathrm{AdS}_{n}\left(\mathrm{H}^{n}\right)$ isometry algebra for a Lorentzian (a Euclidean) brane, respectively. After contraction, the isometry of the transverse space is reduced to the Poincaré algebra iso $(4-m) \times$ iso $(7-n)$ $(\operatorname{iso}(3-m, 1) \times \operatorname{iso}(7-n))$ or iso $(4-m) \times \operatorname{iso}(7-n)(\operatorname{iso}(4-m) \times \operatorname{iso}(6-n, 1))$ for a Lorentzian (a Euclidean) brane. We require that the contracted superalgebra contains a super subalgebra, the supersymmetrization of the direct product of the isometry algebra on the AdS brane worldvolume and the Lorentz symmetry in the transverse space, so $(m-$ $1,2) \times \mathrm{so}(n+1) \times \mathrm{so}(4-m) \times \mathrm{so}(7-n)(\mathrm{so}(m, 1) \times \mathrm{so}(n+1) \times \mathrm{so}(3-m, 1) \times \mathrm{so}(7-n))$ for a Lorentzian (a Euclidean) brane in $\mathrm{AdS}_{4} \times \mathrm{S}^{7}$, and so $(m+1) \times \operatorname{so}(n-1,2) \times \operatorname{so}(4-m) \times \operatorname{so}(7-$ $n)(\operatorname{so}(m+1) \times \operatorname{so}(n, 1) \times \operatorname{so}(4-m) \times \operatorname{so}(6-n, 1))$ for a Lorentzian (a Euclidean) brane in $\mathrm{S}^{4} \times \mathrm{AdS}_{7}$, respectively. This is satisfied if

$$
M^{\prime} \Gamma^{\bar{A}}=\Gamma^{\bar{A}} M, \quad M^{\prime} \widehat{\Gamma}^{\bar{A} \bar{B}}=\widehat{\Gamma}^{\bar{A} \bar{B}} M
$$

where

$$
M^{\prime}=C^{-1} M^{T} C=(-1)^{p+1+\left[\frac{p+1}{2}\right]} M
$$

The first condition is satisfied if $p=1,2 \bmod 4$. Since

$$
M^{\prime} \widehat{\Gamma}^{\bar{A} \bar{B}}=(-1)^{p+1+\mathrm{d}+\left[\frac{p+1}{2}\right]} \widehat{\Gamma}^{\bar{A} \bar{B}} M
$$

where $\mathrm{d}$ is the number of the Dirichlet directions contained in $\{\sharp, 1,2,3\}$, these are satisfied by (odd,odd)-branes $(p=1 \bmod 4)$ and (even,even)-branes $(p=3 \bmod 4)$. We depict branes in Table 4 . The 10 -brane is just $\mathrm{AdS}_{4 / 7} \times \mathrm{S}^{7 / 4}$ itself as $M=1$. The $p$-branes with 
$p=1 \bmod 4$ are $1 / 2$ BPS Dirichlet branes of an open supermembrane in $\mathrm{AdS}_{4 / 7} \times \mathrm{S}^{7 / 4}$ $[26,27]$. The brane probe analysis for M-branes [42] is also consistent with this result.

We derive the NH superalgebra for these branes as IW contractions of the super$\mathrm{AdS}_{4 / 7} \times \mathrm{S}^{7 / 4}$ algebra. First, we rescale generators as

$$
P_{\underline{A}} \rightarrow \frac{1}{\Omega} P_{\underline{A}}, \quad J_{\bar{A} \underline{B}} \rightarrow \frac{1}{\Omega} J_{\bar{A} \underline{B}}, \quad Q_{-} \rightarrow \frac{1}{\Omega} Q_{-}
$$

where we have decomposed $Q$ as

$$
Q=Q_{+}+Q_{-}, \quad Q_{ \pm} \mathcal{P}_{ \pm}=Q_{ \pm}, \quad \mathcal{P}_{ \pm}=\frac{1}{2}(1+M)
$$

Substituting these into (6.1) and then taking the limit $\Omega \rightarrow 0$, we derive the NH superalgebra for an AdS brane

$$
\begin{aligned}
& {\left[P_{\bar{a}}, P_{\bar{b}}\right]=4 \epsilon^{2} \lambda^{2} J_{\bar{a} \bar{b}}, \quad\left[P_{\bar{a}^{\prime}}, P_{\bar{b}^{\prime}}\right]=-\epsilon^{2} \lambda^{2} J_{\bar{a}^{\prime} \bar{b}^{\prime}},} \\
& {\left[P_{\bar{a}}, P_{\underline{b}}\right]=4 \epsilon^{2} \lambda^{2} J_{\bar{a} \underline{b}}, \quad\left[P_{\bar{a}^{\prime}}, P_{\underline{b}^{\prime}}\right]=-\epsilon^{2} \lambda^{2} J_{\bar{a}^{\prime} \underline{b}^{\prime}},} \\
& {\left[J_{\bar{A} \bar{B}}, P_{\bar{C}}\right]=\eta_{\bar{B} \bar{C}} P_{\bar{A}}-\eta_{\bar{A} \bar{C}} P_{\bar{B}}, \quad\left[J_{\bar{A} \underline{B}}, P_{\bar{C}}\right]=-\eta_{\bar{A} \bar{C}} P_{\underline{B}},} \\
& {\left[J_{\underline{A B}}, P_{\underline{C}}\right]=\eta_{\underline{B} \bar{C}} P_{\underline{A}}-\eta_{\underline{A C}} P_{\underline{B}},} \\
& {\left[J_{\bar{A} \bar{B}}, J_{\bar{C} \bar{D}}\right]=\eta_{\bar{B} \bar{C}} J_{\bar{A} \bar{D}}+3 \text {-terms }, \quad\left[J_{\underline{A B}}, J_{\underline{C D}}\right]=\eta_{\underline{B C}} J_{\underline{A D}}+3 \text {-terms }} \\
& {\left[J_{\bar{A} \bar{B}}, J_{\bar{C} \underline{D}}\right]=\eta_{\bar{B} \bar{C}} J_{\bar{A} \underline{D}}-\eta_{\bar{A} \bar{C}} J_{\bar{B} \underline{D}}, \quad\left[J_{\underline{A B}}, J_{\bar{C} \underline{D}}\right]=\eta_{\underline{B D}} J_{\bar{C} \underline{A}}-\eta_{\underline{A D}} J_{\bar{C} \underline{B}},} \\
& {\left[P_{\bar{A}}, Q_{ \pm}\right]=-\frac{\lambda}{2} Q_{ \pm} \widehat{\Gamma}_{\bar{A}}, \quad\left[P_{\underline{A}}, Q_{+}\right]=-\frac{\hat{A}}{2} Q_{-} \widehat{\Gamma}_{\underline{A}},} \\
& {\left[J_{\bar{A} \bar{B}}, Q_{ \pm}\right]=\frac{1}{2} Q_{ \pm} \Gamma_{\bar{A} \bar{B}}, \quad\left[J_{\underline{A B}}, Q_{ \pm}\right]=\frac{1}{2} Q_{ \pm} \Gamma_{\underline{A B}}, \quad\left[J_{\bar{A} \underline{B}}, Q_{+}\right]=\frac{1}{2} Q_{-} \Gamma_{\bar{A} \underline{B}},} \\
& \left\{Q_{+}, Q_{+}\right\}=-2 \mathcal{C} \Gamma^{\bar{A}} \mathcal{P}_{+} P_{\bar{A}}+\lambda \mathcal{C} \widehat{\Gamma}^{\bar{A} \bar{B}} \mathcal{P}_{+} J_{\bar{A} \bar{B}}+\lambda \mathcal{C} \widehat{\Gamma}_{\underline{A B}} \mathcal{P}_{+} J_{\underline{A B}}, \\
& \left\{Q_{+}, Q_{-}\right\}=-2 \mathcal{C} \Gamma^{A} \mathcal{P}_{-} P_{\underline{A}}+2 \lambda \mathcal{C} \widehat{\Gamma}^{\bar{A} \underline{B}} \mathcal{P}_{-} J_{\bar{A} \underline{B}} .
\end{aligned}
$$

We note that this superalgebra contains two bosonic algebras and a superalgebra as subalgebras. One is the isometry of the $(m, n)$-brane worldvolume, generated by $\left\{P_{\bar{A}}, J_{\bar{A} \bar{B}}\right\}$, the $\operatorname{AdS}_{m}\left(\mathrm{H}^{m}\right) \times \mathrm{S}^{n}$ algebra for a Lorentzian (a Euclidean) brane in $\mathrm{AdS}_{4} \times \mathrm{S}^{7}$ and the $\mathrm{S}^{m} \times \operatorname{AdS}_{n}\left(\mathrm{H}_{n}\right)$ algebra for a Lorentzian (a Euclidean) brane in $\mathrm{S}^{4} \times \mathrm{AdS}_{7}$. Another is the isometry in the transverse space generated by $\left\{P_{\underline{A}}, J_{\underline{A B}}\right\}$, the Poincaré algebra $\operatorname{iso}(4-m) \times \operatorname{iso}(7-n)(\operatorname{iso}(3-m, 1) \times \operatorname{iso}(7-n))$ in $\operatorname{AdS}_{4} \times \mathrm{S}^{7}$ and iso $(4-m) \times \operatorname{iso}(7-n)$ ( iso $(4-m) \times \operatorname{iso}(6-n, 1))$ in $\mathrm{S}^{4} \times \mathrm{AdS}_{7}$ for a Lorentzian (a Euclidean) brane. The other 
is the superalgebra generated by $\left\{P_{\bar{A}}, J_{\bar{A} \bar{B}}, J_{\underline{A B}}, Q_{+}\right\}$

$$
\begin{aligned}
& {\left[P_{\bar{a}}, P_{\bar{b}}\right]=4 \epsilon^{2} \lambda^{2} J_{\bar{a} \bar{b}}, \quad\left[P_{\bar{a}^{\prime}}, P_{\bar{b}^{\prime}}\right]=-\epsilon^{2} \lambda^{2} J_{\bar{a}^{\prime} \bar{b}^{\prime}}, \quad\left[J_{\bar{A} \bar{B}}, P_{\bar{C}}\right]=\eta_{\bar{B} \bar{C}} P_{\bar{A}}-\eta_{\bar{A} \bar{C}} P_{\bar{B}},} \\
& {\left[J_{\bar{A} \bar{B}}, J_{\bar{C} \bar{D}}\right]=\eta_{\bar{B} \bar{C}} J_{\bar{A} \bar{D}}+3 \text {-terms }, \quad\left[J_{\underline{A B}}, J_{\underline{C D}}\right]=\eta_{\underline{B C}} J_{\underline{A D}}+3 \text {-terms },} \\
& {\left[P_{\bar{A}}, Q_{+}\right]=-\frac{\lambda}{2} Q_{+} \widehat{\Gamma}_{\bar{A}}, \quad\left[J_{\bar{A} \bar{B}}, Q_{+}\right]=\frac{1}{2} Q_{+} \Gamma_{\bar{A} \bar{B}}, \quad\left[J_{\underline{A B}}, Q_{+}\right]=\frac{1}{2} Q_{+} \Gamma_{\underline{A B}},} \\
& \left\{Q_{+}, Q_{+}\right\}=-2 \mathcal{C} \Gamma^{\bar{A}} \mathcal{P}_{+} P_{\bar{A}}+\lambda \hat{C} \widehat{\Gamma}^{\bar{A} \bar{B}} \mathcal{P}_{+} J_{\bar{A} \bar{B}}+\lambda \mathcal{C} \widehat{\Gamma}^{\underline{A B}} \mathcal{P}_{+} J_{\underline{A B}},
\end{aligned}
$$

which is the supersymmetrization of the algebra, $\operatorname{so}(m-1,2) \times \operatorname{so}(n+1) \times \operatorname{so}(4-m) \times \operatorname{so}(7-$ $n)(\operatorname{so}(m, 1) \times \operatorname{so}(n+1) \times \operatorname{so}(3-m, 1) \times \operatorname{so}(7-n))$ for a Lorentzian (a Euclidean) brane in $\mathrm{AdS}_{4} \times \mathrm{S}^{7}$, and $\mathrm{so}(m+1) \times \operatorname{so}(n-1,2) \times \operatorname{so}(4-m) \times \operatorname{so}(7-n)(\operatorname{so}(m+1) \times \operatorname{so}(n, 1) \times \operatorname{so}(4-$ $m) \times \operatorname{so}(6-n, 1))$ for a Lorentzian (a Euclidean) brane in $\mathrm{S}^{4} \times \mathrm{AdS}_{7}$. For a $(4,7)$-brane the superalgebra is obviously $\operatorname{osp}(8 \mid 4)$ or $\operatorname{osp}\left(8^{*} \mid 4\right)$. Since the dimension of the bosonic subalgebra is 18 for $(0,3)$ - and (3,3)-branes, 20 for $(1,1)$-, (2,1)-, (1,5)- and (2,5)-branes, 22 for a (4,3)-brane, and 34 for $(0,7)$ - and (3,7)-branes, one may guess the superalgebra as those including variants of $\operatorname{osp}(4 \mid 2) \times \operatorname{osp}(4 \mid 2), \operatorname{osp}(6 \mid 2) \times \operatorname{so}(2 \mid 2), \operatorname{sp}(4 \mid 2) \times \operatorname{osp}(4 \mid 2)$ and $\operatorname{osp}(8 \mid 2) \times \operatorname{su}(2)$, respectively. The existence of this superalgebra is ensured by (6.7).

The $\mathrm{NH}$ superalgebra (6.12) is equivalent to the MC equation

$$
\begin{aligned}
& d \mathbf{L}^{\bar{A}}=-\eta_{\bar{B} \bar{C}} \mathbf{L}^{\bar{A} \bar{B}} \mathbf{L}^{\bar{C}}-\bar{L}_{+} \Gamma^{\bar{A}} L_{+}, \\
& d \mathbf{L}^{\underline{A}}=-\eta_{\bar{B} \bar{C}} \mathbf{L}^{\underline{A}} \underline{\bar{B}} \mathbf{L}^{\bar{C}}-\eta_{\underline{B C}} \mathbf{L}^{\underline{A B}} \mathbf{L}^{\underline{C}}-\bar{L}_{+} \Gamma^{\underline{A}} L_{-}-\bar{L}_{-} \Gamma^{\underline{A}} L_{+}, \\
& d \mathbf{L}^{\bar{a} \bar{b}}=-4 \epsilon^{2} \lambda^{2} \mathbf{L}^{\bar{a}} \mathbf{L}^{\bar{b}}-\eta_{\bar{c} \bar{d}} \mathbf{L}^{\bar{c} \bar{a}} \mathbf{L}^{\bar{b} \bar{d}}+2 \lambda \bar{L}_{+} \mathcal{I} \Gamma^{\bar{a} \bar{b}} L_{+}, \\
& d \mathbf{L}^{\bar{a}^{\prime} \bar{b}^{\prime}}=+\lambda^{2} \mathbf{L}^{\bar{a}^{\prime}} \mathbf{L}^{\bar{b}^{\prime}}-\eta_{\bar{c}^{\prime} \bar{d}^{\prime}} \mathbf{L}^{\bar{c}^{\prime} \bar{a}^{\prime}} \mathbf{L}^{\bar{b}^{\prime} \bar{d}^{\prime}}-\lambda \bar{L}_{+} \mathcal{I} \Gamma^{\bar{a}^{\prime} \bar{b}^{\prime}} L_{+}, \\
& d \mathbf{L} \underline{\underline{A B}}=-\eta_{\underline{C D}} \mathbf{L}^{\underline{C D}} \mathbf{L} \frac{B D}{\underline{B D}}+\lambda \bar{L}_{+} \widehat{\Gamma} \underline{\underline{A B}} L_{+}, \\
& d \mathbf{L}^{\bar{a} \underline{b}}=-4 \epsilon^{2} \lambda^{2} \mathbf{L}^{\bar{a}} \mathbf{L}^{\underline{b}}-\eta_{\bar{c} \bar{d}} \mathbf{L}^{\bar{c} \bar{a}} \mathbf{L}^{\underline{b} \bar{d}}-\eta_{\underline{c} \underline{ }} \mathbf{L}^{\underline{\underline{c} a \bar{a}}} \mathbf{L}^{\underline{b d}} \\
& +2 \lambda \bar{L}_{+} \mathcal{I} \Gamma^{\bar{a} \underline{b}} L_{-}+2 \lambda \bar{L}_{-} \mathcal{I} \Gamma^{\bar{a} \underline{b}} L_{+}, \\
& d \mathbf{L}^{\bar{a}^{\prime} \underline{\underline{b}^{\prime}}}=+\epsilon^{2} \lambda^{2} \mathbf{L}^{\bar{a}^{\prime}} \mathbf{L}^{\underline{b}^{\prime}}-\eta_{\bar{c}^{\prime} \bar{d}^{\prime}} \mathbf{L}^{\mathbf{L}^{\prime} \bar{a}^{\prime}} \mathbf{L}^{\underline{b}^{\prime} \bar{d}^{\prime}}-\eta_{\underline{c}^{\prime} \underline{d}^{\prime}} \mathbf{L}^{\underline{c}^{\prime} \bar{a}^{\prime}} \mathbf{L}^{\underline{b^{\prime}} \underline{d}^{\prime}} \\
& -\lambda \bar{L}_{+} \mathcal{I} \Gamma^{\bar{a}^{\prime} \underline{b}^{\prime}} L_{-}-\lambda \bar{L}_{-} \mathcal{I} \Gamma^{\bar{a}^{\prime} \underline{b}^{\prime}} L_{+}, \\
& d L_{+}=\frac{\lambda}{2} \mathbf{L}^{\bar{A}} \widehat{\Gamma}_{\bar{A}} L_{+}-\frac{1}{4} \mathbf{L}^{\bar{A} \bar{B}} \Gamma_{\bar{A} \bar{B}} L_{+}-\frac{1}{4} \mathbf{L}^{\underline{A B}} \Gamma_{\underline{A B}} L_{+}, \\
& d L_{-}=\frac{\lambda}{2} \mathbf{L}^{\bar{A}} \widehat{\Gamma}_{\bar{A}} L_{-} \frac{\lambda}{2} \mathbf{L} \underline{A}^{\underline{\Gamma_{A}}} L_{+} \\
& -\frac{1}{4} \mathbf{L}^{\bar{A} \bar{B}} \Gamma_{\bar{A} \bar{B}} L_{-}-\frac{1}{4} \mathbf{L}^{\underline{A B}} \Gamma_{\underline{A B}} L_{-}-\frac{1}{2} \mathbf{L}^{\bar{A} \underline{B}} \Gamma_{\bar{A} \underline{B}} L_{+} .
\end{aligned}
$$

The MC equation above can be obtained by rescaling Cartan one-forms in (6.5) as

$$
\mathbf{L}^{\underline{A}} \rightarrow \Omega \mathbf{L}^{\underline{A}}, \quad \mathbf{L}^{\bar{A} \underline{B}} \rightarrow \Omega \mathbf{L}^{\bar{A} \underline{B}}, \quad L_{-} \rightarrow \Omega L_{-},
$$


and taking the limit $\Omega \rightarrow 0$.

\section{$7 \quad \mathrm{NH}$ superalgebra of Branes in M pp-wave}

We define

$$
\begin{aligned}
& P_{ \pm}=\frac{1}{\sqrt{2}}\left(P_{\natural} \pm P_{0}\right), \quad P_{\hat{i}}^{*}=\left(P_{i}^{*}=\left\{\begin{array} { l } 
{ J _ { i 0 } } \\
{ J _ { i \natural } }
\end{array} , P _ { i ^ { \prime } } ^ { * } = \{ \begin{array} { c } 
{ J _ { i ^ { \prime } \natural } } \\
{ J _ { i ^ { \prime } 0 } }
\end{array} ) \quad \text { for } \quad \left\{\begin{array}{l}
\operatorname{AdS}_{4} \times \mathrm{S}^{7} \\
\operatorname{AdS}_{7} \times \mathrm{S}^{4}
\end{array}\right.\right.\right. \\
& Q^{( \pm)}=Q^{( \pm)} \ell_{ \pm}, \quad \ell_{ \pm}=\frac{1}{2} \Gamma_{ \pm} \Gamma_{\mp}, \quad \Gamma_{ \pm}=\frac{1}{\sqrt{2}}\left(\Gamma_{\natural} \pm \Gamma_{0}\right), \\
& \mathcal{I}=\Gamma^{\sharp 123}, \quad \Gamma^{\sharp}=\left\{\begin{array} { l } 
{ \Gamma ^ { 0 } } \\
{ - \Gamma ^ { \natural } }
\end{array} \quad \text { for } \left\{\begin{array}{l}
\mathrm{AdS}_{4} \times \mathrm{S}^{7} \\
\mathrm{AdS}_{7} \times \mathrm{S}^{4}
\end{array}\right.\right.
\end{aligned}
$$

where where $i=1,2,3$ and $i^{\prime}=4,5,6,7,8,9$.

Scaling generators in the super- $\mathrm{AdS}_{4 / 7} \times \mathrm{S}^{7 / 4}$ algebra as

$$
P_{+} \rightarrow \frac{1}{\Lambda^{2}} P_{+}, \quad P_{\hat{i}} \rightarrow \frac{1}{\Lambda} P_{\hat{i}}, \quad P_{\hat{i}}^{*} \rightarrow \frac{1}{\Lambda} P_{\hat{i}}^{*}, \quad Q^{(+)} \rightarrow \frac{1}{\Lambda} Q^{(+)}
$$

and taking the limit $\Lambda \rightarrow 0$ limit [6], we obtain the M pp-wave superalgebra

$$
\begin{aligned}
& {\left[P_{-}, P_{i}\right]=\frac{4 \lambda^{2}}{\sqrt{2}} P_{i}^{*}, \quad\left[P_{-}, P_{i^{\prime}}\right]=\frac{\lambda^{2}}{\sqrt{2}} P_{i^{\prime}}^{*}, \quad\left[P_{-}, P_{\hat{i}}^{*}\right]=-\frac{1}{\sqrt{2}} P_{\hat{i}},} \\
& {\left[P_{\hat{i}}, P_{\hat{j}}^{*}\right]=\frac{1}{\sqrt{2}} \eta_{\hat{i} \hat{j}} P_{+}, \quad\left[P_{\hat{i}}, J_{\hat{j} \hat{k}}\right]=\eta_{\hat{i} \hat{j}} P_{\hat{k}}-\eta_{\hat{i} \hat{k}} P_{\hat{j}}, \quad\left[P_{\hat{i}}^{*}, J_{\hat{j} \hat{k}}\right]=\eta_{\hat{i} \hat{j}} P_{\hat{k}}^{*}-\eta_{\hat{i} \hat{k}} P_{\hat{j}}^{*},} \\
& {\left[J_{\hat{i} \hat{j}}, J_{\hat{k} \hat{l}}\right]=\eta_{\hat{j} \hat{k}} J_{\hat{i} \hat{l}}+3 \text {-terms },} \\
& {\left[P_{-}, Q^{(+)}\right]=-\frac{3 \lambda}{2 \sqrt{2}} Q^{(+)} f, \quad\left[P_{-}, Q^{(-)}\right]=-\frac{\lambda}{2 \sqrt{2}} Q^{(-)} f,} \\
& {\left[P_{i}, Q^{(-)}\right]=-\frac{\lambda}{\sqrt{2}} Q^{(+)} f \Gamma_{i} \Gamma_{+}, \quad\left[P_{i^{\prime}}, Q^{(-)}\right]=-\frac{\lambda}{2 \sqrt{2}} Q^{(+)} f \Gamma_{i^{\prime}} \Gamma_{+},} \\
& {\left[J_{\hat{i} \hat{j}}, Q^{( \pm)}\right]=\frac{1}{2} Q^{( \pm)} \Gamma_{\hat{i} \hat{j}}, \quad\left[P_{\hat{i}}^{*}, Q^{(-)}\right]=\frac{1}{2 \sqrt{2}} Q^{(+)} \Gamma_{\hat{i}} \Gamma_{+},} \\
& \left\{Q^{(+)}, Q^{(+)}\right\}=-2 \mathcal{C} \Gamma_{-} P_{+}, \\
& \left\{Q^{(-)}, Q^{(-)}\right\}=-2 \mathcal{C} \Gamma_{+} P_{-}-\frac{\lambda}{\sqrt{2}} \mathcal{C} \widehat{\Gamma}^{\hat{i} \hat{j}} J_{\hat{i} \hat{j}} \\
& \left\{Q^{( \pm)}, Q^{(\mp)}\right\}=-2 \mathcal{C} \Gamma^{\hat{i}} \ell_{\mp} P_{\hat{i}}-4 \lambda \mathcal{C} f \Gamma^{i} \ell_{\mp} P_{i}^{*} \mp 2 \lambda \mathcal{C} f \Gamma^{i^{\prime}} \ell_{\mp} P_{i^{\prime}}^{*}
\end{aligned}
$$

where $\widehat{\Gamma}^{\hat{i} \hat{j}}=\left(-2 \Gamma_{+} f \Gamma^{i j}, \Gamma_{+} f \Gamma^{i^{\prime} j^{\prime}}\right)$ and $f=\Gamma^{123}$. The bosonic subalgebra is the semi-direct product of the Heisenberg algebra generated by $\left\{P_{\hat{i}}, P_{\hat{i}}^{*}\right\}$ with an outer automorphism $P_{-}$ and the Lorentz symmetry generated by $J_{\hat{i} \hat{j}}$. 


\subsection{Lorentzian branes}

We consider a Lorentzian pp-wave brane for which $(+,-)$ directions are contained in the Neumann directions. We denote Neumann and Dirichlet directions, $\bar{A}=(+,-\overline{\hat{i}})$ and $\underline{A}=\underline{\hat{i}}$, respectively.

We derive NH superalgebras of Lorentzian pp-wave branes as IW contractions of the super- $\mathrm{AdS}_{4 / 7} \times \mathrm{S}^{7 / 4}$ algebra.

First we consider the bosonic subalgebra. The contraction is taken by rescaling generators as

$$
P_{\underline{A}} \rightarrow \frac{1}{\Omega} P_{\underline{A}}, \quad J_{\bar{A} \underline{B}} \rightarrow \frac{1}{\Omega} J_{\bar{A} \underline{B}}, \quad P_{\underline{\hat{i}}}^{*} \rightarrow \frac{1}{\Omega} P_{\underline{\hat{i}}}^{*}
$$

and taking the limit $\Omega \rightarrow 0$. One obtains the NH algebra of an M pp-wave brane

$$
\begin{aligned}
& {\left[P_{-}, P_{i}\right]=\frac{4 \lambda^{2}}{\sqrt{2}} P_{\bar{i}}^{*}, \quad\left[P_{-}, P_{\underline{i}}\right]=\frac{4 \lambda^{2}}{\sqrt{2}} P_{\underline{i}}^{*}, \quad\left[P_{-}, P_{i^{\prime}}\right]=\frac{\lambda^{2}}{\sqrt{2}} P_{\bar{i}^{\prime}}^{*}, \quad\left[P_{-}, P_{\underline{i}^{\prime}}\right]=\frac{\lambda^{2}}{\sqrt{2}} P_{\underline{i}^{\prime}}^{*},} \\
& {\left[P_{-}, P_{\hat{\hat{i}}}^{*}\right]=-\frac{1}{\sqrt{2}} P_{\hat{\hat{i}}}, \quad\left[P_{-}, P_{\underline{\hat{i}}}^{*}\right]=-\frac{1}{\sqrt{2}} P_{\underline{\hat{i}}}, \quad\left[P_{\hat{i}}, P_{\hat{\hat{j}}}^{*}\right]=\frac{1}{\sqrt{2}} \eta_{\hat{i} \hat{j}} P_{+},} \\
& {\left[P_{\overline{\hat{i}}}, J_{\overline{\hat{j}} \hat{\hat{k}}}\right]=\eta_{\hat{\hat{i}} \overline{\hat{j}}} P_{\underline{\hat{k}}}, \quad\left[P_{\overline{\hat{i}}}^{*}, J_{\hat{\bar{j}} \underline{\hat{k}}}\right]=\eta_{\hat{\hat{i}} \overline{\hat{j}}} P_{\underline{\hat{k}}}^{*},}
\end{aligned}
$$

and

$$
\begin{aligned}
& {\left[P_{\overline{\hat{i}}}, J_{\overline{\hat{j}} \overline{\hat{k}}}\right]=\eta_{\bar{i} \overline{\hat{j}}} P_{\hat{\hat{k}}}-\eta_{\overline{\hat{i}} \overline{\hat{k}}} P_{\overline{\hat{j}}}, \quad\left[P_{\underline{\hat{i}}}, J_{\underline{\hat{j}} \underline{\hat{k}}}\right]=\eta_{\underline{\hat{i}} \underline{j}} P_{\underline{\hat{k}}}-\eta_{\underline{\hat{i}} \underline{\hat{k}}} P_{\hat{j}},} \\
& {\left[P_{\hat{i}}^{*}, J_{\overline{\hat{j}} \hat{\hat{k}}}\right]=\eta_{\overline{\hat{i}} \overline{\hat{j}}} P_{\hat{\hat{k}}}^{*}-\eta_{\overline{\hat{i}} \overline{\hat{k}}} P_{\overline{\hat{j}}}^{*}, \quad\left[P_{\underline{\hat{i}}}^{*}, J_{\underline{\hat{j}} \underline{\hat{k}}}\right]=\eta_{\underline{\hat{i} \hat{j}}} P_{\underline{\hat{k}}}^{*}-\eta_{\underline{\hat{i} \hat{k}}} P_{\underline{\hat{j}}}^{*},} \\
& {\left[J_{\overline{\hat{i} \bar{j}}}, J_{\overline{\hat{k}} \overline{\hat{l}}}\right]=\eta_{\overline{\hat{j}} \overline{\hat{k}}} J_{\overline{\hat{i}} \overline{\hat{l}}}+3 \text {-terms }, \quad\left[J_{\underline{\hat{i} \hat{j}}}, J_{\underline{\hat{k} \hat{l}}}\right]=\eta_{\underline{\hat{j}} \underline{\hat{k}}} J_{\underline{\hat{i} \hat{l}}}+3 \text {-terms },}
\end{aligned}
$$

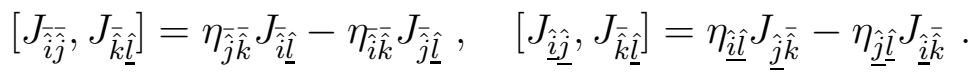

Next we consider the fermionic part. We decompose $Q^{(\bullet)}$ as

$$
Q_{ \pm}^{(\bullet)}= \pm Q_{ \pm}^{(\bullet)} M \quad \text { with } \quad M=\ell \Gamma^{+-\bar{A}_{1} \cdots \bar{A}_{p-1}}, \quad M^{2}=\ell^{2}(-1)^{\left[\frac{p-1}{2}\right]}=1
$$

which satisfies

$$
M^{\prime}=\mathcal{C}^{-1} M^{T} \mathcal{C}=(-1)^{p+1+\left[\frac{p+1}{2}\right]} M
$$

We demand that

$$
\begin{aligned}
& M^{\prime} \Gamma^{\bar{A}}=\Gamma^{\bar{A}} M, \\
& M^{\prime} \widehat{\Gamma}^{\overline{\hat{i}} \overline{\hat{j}}}=\widehat{\Gamma}^{\widehat{\bar{i}} \overline{\hat{j}}} M .
\end{aligned}
$$


Since

$$
M^{\prime} \Gamma^{\bar{A}}=(-1)^{1+\left[\frac{p+1}{2}\right]} \Gamma^{\bar{A}} M
$$

the first condition (7.10) is satisfied when $p=1,2 \bmod 4$. The second condition (7.11) restricts the directions along which a pp-wave brane extends. Since

$$
M^{\prime} \widehat{\Gamma}^{\overline{\hat{i}} \overline{\hat{j}}}=(-1)^{1+\left[\frac{p+1}{2}\right]+\mathrm{n}} \widehat{\Gamma}^{\overline{\hat{i}} \overline{\hat{j}}} M
$$

where $\mathrm{n}$ is the number of the Neumann directions contained in $\{1,2,3\}$, we find that $\mathrm{n}=$ even for $p=1,2 \bmod 4$. In Table 5 we summarize the result. The $p$-branes with $p=1 \bmod 4$ are Dirichlet branes of an open supermembrane in $\mathrm{M}$ pp-wave $[46,47]$.

\begin{tabular}{|c|c|c|c|c|c|}
\hline 1-brane & 2-brane & 5-brane & 6-brane & 9-brane & 10-brane \\
\hline$(+,-)$ & $(+,-; 0,1)$ & $\begin{array}{c}(+,-; 0,4) \\
(+,-; 2,2)\end{array}$ & $\begin{array}{c}(+,-; 0,5) \\
(+,-; 2,3)\end{array}$ & $(+,-; 2,6)$ & - \\
& & & \\
\hline
\end{tabular}

Table 5: Lorentzian M pp-wave branes

Scaling $Q_{ \pm}^{(\bullet)}$ as

$$
Q_{-}^{(\bullet)} \rightarrow \frac{1}{\Omega} Q_{-}^{(\bullet)}
$$

and taking the limit $\Omega \rightarrow 0$, we obtain the fermionic part of the NH superalgebra

$$
\begin{aligned}
& {\left[P_{-}, Q_{ \pm}^{(+)}\right]=-\frac{3 \lambda}{2 \sqrt{2}} Q_{ \pm}^{(+)} f, \quad\left[P_{-}, Q_{ \pm}^{(-)}\right]=-\frac{\lambda}{2 \sqrt{2}} Q_{ \pm}^{(-)} f} \\
& {\left[P_{\bar{i}}, Q_{ \pm}^{(-)}\right]=-\frac{\lambda}{\sqrt{2}} Q_{ \pm}^{(+)} f \Gamma_{\bar{i}} \Gamma_{+}, \quad\left[P_{\underline{i}}, Q_{+}^{(-)}\right]=-\frac{\lambda}{\sqrt{2}} Q_{-}^{(+)} f \Gamma_{\underline{i}} \Gamma_{+},} \\
& {\left[P_{i^{\prime}}, Q_{ \pm}^{(-)}\right]=-\frac{\lambda}{2 \sqrt{2}} Q_{ \pm}^{(+)} f \Gamma_{\bar{i}^{\prime}} \Gamma_{+}, \quad\left[P_{\underline{i}^{\prime}}, Q_{+}^{(-)}\right]=-\frac{\lambda}{2 \sqrt{2}} Q_{-}^{(+)} f \Gamma_{\underline{i}^{\prime}} \Gamma_{+},}
\end{aligned}
$$

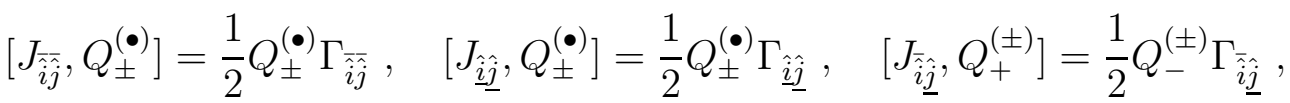

$$
\begin{aligned}
& {\left[P_{\hat{i}}^{*}, Q_{ \pm}^{(-)}\right]=\frac{1}{2 \sqrt{2}} Q_{ \pm}^{(+)} \Gamma_{\overline{\hat{i}}} \Gamma_{+}, \quad\left[P_{\underline{\hat{i}}}^{*}, Q_{+}^{(-)}\right]=\frac{1}{2 \sqrt{2}} Q_{-}^{(+)} \Gamma_{\underline{\hat{i}}} \Gamma_{+},} \\
& \left\{Q_{+}^{(+)}, Q_{+}^{(+)}\right\}=-2 \mathcal{C} \Gamma_{-} \mathcal{P}_{+} P_{+} \\
& \left\{Q_{+}^{(-)}, Q_{+}^{(-)}\right\}=-2 \mathcal{C} \Gamma_{+} \mathcal{P}_{+} P_{-}+\frac{\lambda}{\sqrt{2}} \mathcal{C} \widehat{\Gamma}^{\bar{i} \overline{\hat{j}}} \mathcal{P}_{+} J_{\overline{\hat{i}} \overline{\hat{j}}}+\frac{\lambda}{\sqrt{2}} \mathcal{C} \widehat{\Gamma} \stackrel{\hat{i} \hat{j}}{-} \mathcal{P}_{+} J_{\underline{\underline{i}} \hat{j}}, \\
& \left\{Q_{ \pm}^{(-)}, Q_{\mp}^{(-)}\right\}=\sqrt{2} \lambda \mathcal{C} \widehat{\Gamma}^{\overline{\overline{\hat{i}}} \underline{\hat{j}}} J_{\overline{\hat{i} \hat{j}}}, \\
& \left\{Q_{+}^{( \pm)}, Q_{+}^{(\mp)}\right\}=-2 \mathcal{C} \Gamma^{\overline{\hat{i}}} \ell_{\mp} \mathcal{P}_{+} P_{\hat{i}}-4 \lambda \mathcal{C} f \Gamma^{\bar{i}} \ell_{\mp} \mathcal{P}_{+} P_{\bar{i}}^{*} \mp 2 \lambda \mathcal{C} f \Gamma^{\bar{i}^{\prime}} \ell_{\mp} \mathcal{P}_{+} P_{\bar{i}^{\prime}}^{*} \\
& \left\{Q_{+}^{( \pm)}, Q_{-}^{(\mp)}\right\}=-2 \mathcal{C} \Gamma^{\underline{\hat{i}}} \ell_{\mp} \mathcal{P}_{-} P_{\underline{\hat{i}}}-4 \lambda \mathcal{C} f \Gamma^{\underline{i}} \ell_{\mp} \mathcal{P}_{-} P_{\underline{i}}^{*} \mp 2 \lambda \mathcal{C} f \Gamma^{i^{\prime}} \ell_{\mp} \mathcal{P}_{-} P_{\underline{i}^{\prime}}^{*}
\end{aligned}
$$


Summarizing we have derived the NH superalgebra of an M pp-wave brane as (7.6),$(7.7)$ and (7.15).

We note that the NH superalgebra of a Lorentzian M pp-wave brane contains a supersubalgebra generated by $P_{ \pm}, P_{\hat{\hat{i}}}, P_{\overline{\hat{i}}}^{*}, J_{\overline{\hat{i}} \overline{\hat{j}}}, J_{\underline{\hat{i} \hat{j}}}$ and $Q_{+}^{( \pm)}$

$$
\begin{aligned}
& {\left[P_{-}, P_{i}\right]=\frac{4 \lambda^{2}}{\sqrt{2}} P_{\bar{i}}^{*}, \quad\left[P_{-}, P_{i^{\prime}}\right]=\frac{\lambda^{2}}{\sqrt{2}} P_{\bar{i}^{\prime}}^{*}, \quad\left[P_{-}, P_{\hat{i}}^{*}\right]=-\frac{1}{\sqrt{2}} P_{\hat{i}},} \\
& {\left[P_{\hat{i}}, P_{\hat{j}}^{*}\right]=\frac{1}{\sqrt{2}} \eta_{\hat{i} \hat{j}} P_{+}, \quad\left[P_{\hat{i}}, J_{\overline{\hat{j}} \overline{\hat{k}}}\right]=\eta_{\overline{\hat{i}} \overline{\hat{j}}} P_{\overline{\hat{k}}}-\eta_{\overline{\hat{i}} \overline{\hat{k}}} P_{\overline{\hat{j}}}, \quad\left[P_{\hat{i}}^{*}, J_{\overline{\hat{j}} \overline{\hat{k}}}\right]=\eta_{\bar{i} \overline{\hat{j}}} P_{\hat{\hat{k}}}^{*}-\eta_{\overline{\hat{i}} \overline{\hat{k}}} P_{\hat{\hat{j}}}^{*},} \\
& {\left[J_{\overline{\hat{i}} \overline{\hat{j}}}, J_{\overline{\hat{k}} \bar{l}}\right]=\eta_{\overline{\hat{j}} \overline{\hat{k}}} J_{\overline{\hat{i}} \overline{\hat{l}}}+3 \text {-terms }, \quad\left[J_{\underline{\hat{i}} \underline{\hat{j}}}, J_{\underline{\hat{k} \hat{l}}}\right]=\eta_{\underline{\hat{j}} \underline{\hat{k}}} J_{\underline{\hat{i} \hat{l}}}+3 \text {-terms },} \\
& {\left[P_{-}, Q_{+}^{(+)}\right]=-\frac{3 \lambda}{2 \sqrt{2}} Q_{;}^{(+)} f, \quad\left[P_{-}, Q_{+}^{(-)}\right]=-\frac{\lambda}{2 \sqrt{2}} Q_{+}^{(-)} f,} \\
& {\left[P_{i}, Q_{+}^{(-)}\right]=-\frac{\lambda}{\sqrt{2}} Q_{+}^{(+)} f \Gamma_{\bar{i}} \Gamma_{+}, \quad\left[P_{\bar{i}^{\prime}}, Q_{+}^{(-)}\right]=-\frac{\lambda}{2 \sqrt{2}} Q_{+}^{(+)} f \Gamma_{\bar{i}^{\prime}} \Gamma_{+},} \\
& {\left[J_{\overline{\hat{i}} \overline{\hat{j}}}, Q_{+}^{(\bullet)}\right]=\frac{1}{2} Q_{+}^{(\bullet)} \Gamma_{\overline{\hat{i}} \overline{\hat{j}}}, \quad\left[J_{\underline{\hat{i}} \hat{j}}, Q_{+}^{(\bullet)}\right]=\frac{1}{2} Q_{+}^{(\bullet)} \Gamma_{\underline{\hat{i} \hat{j}}}, \quad\left[P_{\overline{\hat{i}}}^{*}, Q_{+}^{(-)}\right]=\frac{1}{2 \sqrt{2}} Q_{+}^{(+)} \Gamma_{\overline{\hat{i}}} \Gamma_{+},} \\
& \left\{Q_{+}^{(+)}, Q_{+}^{(+)}\right\}=-2 \mathcal{C} \Gamma_{-} \mathcal{P}_{+} P_{+} \text {, } \\
& \left\{Q_{+}^{(-)}, Q_{+}^{(-)}\right\}=-2 \mathcal{C} \Gamma_{+} \mathcal{P}_{+} P_{-}+\frac{\lambda}{\sqrt{2}} \mathcal{C} \widehat{\Gamma}^{\bar{i} \overline{\hat{j}}} \mathcal{P}_{+} J_{\overline{\hat{i}} \bar{j}}+\frac{\lambda}{\sqrt{2}} \mathcal{C} \widehat{\Gamma} \stackrel{\hat{i} \hat{j}}{\mathcal{P}_{+}} J_{\underline{\underline{\hat{i}}} \hat{j}}, \\
& \left\{Q_{+}^{( \pm)}, Q_{+}^{(\mp)}\right\}=-2 \mathcal{C} \Gamma^{\overline{\hat{i}}} \ell_{\mp} \mathcal{P}_{+} P_{\hat{i}}-4 \lambda \mathcal{C} f \Gamma^{\bar{i}} \ell_{\mp} \mathcal{P}_{+} P_{\bar{i}}^{*} \mp 2 \lambda \mathcal{C} f \Gamma^{\bar{i}^{\prime}} \ell_{\mp} \mathcal{P}_{+} P_{\bar{i}^{\prime}}^{*} .
\end{aligned}
$$

This is the supersymmetrization of the pp-wave algebra which is the isometry on the brane worldvolume and the Lorentz symmetry in the transverse space. The conditions (17.10) and (17.11) ensure the existence of this superalgebra.

\subsection{Euclidean branes}

We consider a Euclidean pp-wave brane for which $(+,-)$ directions are contained in the Dirichlet directions. We denote Neumann and Dirichlet directions as $\bar{A}=\overline{\hat{i}}$ and $\underline{A}=(+,-, \underline{\hat{i}})$, respectively.

We derive NH superalgebras of Euclidean pp-wave branes as IW contractions of the super-AdS $\mathrm{A}_{4 / 7} \times \mathrm{S}^{7 / 4}$ algebra. First we consider the bosonic subalgebra. The contraction is taken by rescaling generators as

$$
P_{\underline{A}} \rightarrow \frac{1}{\Omega} P_{\underline{A}}, \quad J_{\bar{A} \underline{B}} \rightarrow \frac{1}{\Omega} J_{\bar{A} \underline{B}}, \quad P_{\overline{\hat{i}}}^{*} \rightarrow \frac{1}{\Omega} P_{\overline{\hat{i}}}^{*},
$$


and taking the limit $\Omega \rightarrow 0$. One obtains the NH algebra of a Euclidean M pp-wave brane

$$
\begin{aligned}
& {\left[P_{-}, P_{i}\right]=\frac{4 \lambda^{2}}{\sqrt{2}} P_{\bar{i}}^{*}, \quad\left[P_{-}, P_{i^{\prime}}\right]=\frac{\lambda^{2}}{\sqrt{2}} P_{\bar{i}^{\prime}}^{*}, \quad\left[P_{-}, P_{\underline{\hat{i}}}^{*}\right]=-\frac{1}{\sqrt{2}} P_{\underline{\hat{i}}}, \quad\left[P_{\hat{i}}, J_{\overline{\hat{j}} \underline{\hat{k}}}\right]=\eta_{\overline{\hat{i}} \overline{\hat{j}}} P_{\underline{\hat{k}}},} \\
& {\left[P_{\hat{i}}, P_{\hat{\hat{j}}}^{*}\right]=\frac{1}{\sqrt{2}} \eta_{\overline{\hat{i} \hat{j}}} P_{+}, \quad\left[P_{\underline{\hat{i}}}, P_{\underline{\hat{j}}}^{*}\right]=\frac{1}{\sqrt{2}} \eta_{\underline{\hat{i} \hat{j}}} P_{+}, \quad\left[P_{\underline{\hat{i}}}^{*}, J_{\overline{\hat{j}} \underline{\hat{k}}}\right]=-\eta_{\underline{\hat{i}} \underline{\hat{k}}} P_{\hat{\hat{j}}}^{*},}
\end{aligned}
$$

and (7.7).

Next we consider the fermionic part of the NH superalgebra. We decompose $Q^{(\bullet)}$ as

$$
Q_{ \pm}^{(\bullet)}= \pm Q_{ \pm}^{(\bullet)} M, \quad M=\ell \Gamma^{\bar{A}_{0} \cdots \bar{A}_{p}}, \quad M^{2}=\ell^{2}(-1)^{\left[\frac{p+1}{2}\right]}=1
$$

We demand that the conditions (7.10) and (7.11) are satisfied. The first condition (7.10) implies that $p=1,2 \bmod 4$ as

$$
M^{\prime} \Gamma^{\bar{A}}=(-1)^{1+\left[\frac{p+1}{2}\right]} \Gamma^{\bar{A}} M
$$

On the other hand, since

$$
M^{\prime} \widehat{\Gamma}^{\overline{\hat{i}} \overline{\hat{j}}}=(-1)^{\mathrm{n}+\left[\frac{p+1}{2}\right]} \widehat{\Gamma}^{\widehat{\Gamma}^{\hat{i}} \overline{\hat{j}}} M
$$

where $\mathrm{n}$ is the number of the Neumann directions contained in $\{1,2,3\}$, the second condition is satisfied when $\mathrm{n}=$ odd for $p=1,2 \bmod 4$. We summarize the result in Table 6. The $p$-branes with $p=1 \bmod 4$ are Dirichlet branes of an open supermembrane in $M$ pp-wave $[46,47]$.

\begin{tabular}{|c|c|c|c|c|c|}
\hline 1-brane & 2-brane & 5-brane & 6-brane & 9-brane & 10-brane \\
\hline$(1,1)$ & $(1,2),(3,0)$ & $(1,5),(3,3)$ & $(1,6),(3,4)$ & - & - \\
\hline
\end{tabular}

Table 6: Euclidean M pp-wave branes

Scaling $Q_{ \pm}^{(\bullet)}$ as (13.17) and taking the limit $\Omega \rightarrow 0$, we obtain the fermionic part of the 
NH superalgebra of a Euclidean M pp-wave brane

$$
\begin{aligned}
& {\left[P_{-}, Q_{+}^{(+)}\right]=-\frac{3 \lambda}{2 \sqrt{2}} Q_{-}^{(+)} f, \quad\left[P_{-}, Q_{+}^{(-)}\right]=-\frac{\lambda}{2 \sqrt{2}} Q_{-}^{(-)} f,} \\
& {\left[P_{\bar{i}}, Q_{ \pm}^{(-)}\right]=-\frac{\lambda}{\sqrt{2}} Q_{ \pm}^{(+)} f \Gamma_{\bar{i}} \Gamma_{+}, \quad\left[P_{\underline{i}}, Q_{+}^{(-)}\right]=-\frac{\lambda}{\sqrt{2}} Q_{-}^{(+)} f \Gamma_{\underline{i}} \Gamma_{+},} \\
& {\left[P_{\bar{i}^{\prime}}, Q_{ \pm}^{(-)}\right]=-\frac{\lambda}{2 \sqrt{2}} Q_{ \pm}^{(+)} f \Gamma_{\bar{i}^{\prime}} \Gamma_{+}, \quad\left[P_{\underline{i}^{\prime}}, Q_{+}^{(-)}\right]=-\frac{\lambda}{2 \sqrt{2}} Q_{-}^{(+)} f \Gamma_{\underline{i}^{\prime}} \Gamma_{+},} \\
& {\left[J_{\overline{\hat{i}} \bar{j}}, Q_{ \pm}^{\bullet}\right]=\frac{1}{2} Q_{ \pm}^{(\bullet)} \Gamma_{\overline{\hat{i}} \overline{\hat{j}}}, \quad\left[J_{\underline{\hat{i} \hat{j}}}, Q_{ \pm}^{\bullet}\right]=\frac{1}{2} Q_{ \pm}^{(\bullet)} \Gamma_{\underline{\hat{i} \hat{j}}}, \quad\left[J_{\overline{\hat{i}} \underline{\hat{j}}}, Q_{+}^{\bullet}\right]=\frac{1}{2} Q_{-}^{(\bullet)} \Gamma_{\overline{\hat{i}} \underline{\hat{j}}},} \\
& {\left[P_{\hat{i}}^{*}, Q_{+}^{(-)}\right]=\frac{1}{2 \sqrt{2}} Q_{-}^{(+)} \Gamma_{\overline{\hat{i}}} \Gamma_{+}, \quad\left[P_{\underline{\hat{i}}}^{*}, Q_{ \pm}^{(-)}\right]=\frac{1}{2 \sqrt{2}} Q_{ \pm}^{(+)} \Gamma_{\underline{\hat{i}}} \Gamma_{+},} \\
& \left\{Q_{ \pm}^{(+)}, Q_{\mp}^{(+)}\right\}=-2 \mathcal{C} \Gamma_{-} \mathcal{P}_{\mp} P_{+} \\
& \left\{Q_{+}^{(-)}, Q_{+}^{(-)}\right\}=-\frac{\lambda}{\sqrt{2}} \mathcal{C} \widehat{\Gamma}^{\overline{\hat{i}} \overline{\hat{j}}} J_{\overline{\hat{i}} \overline{\hat{j}}}-\frac{\lambda}{\sqrt{2}} \mathcal{C} \widehat{\Gamma}^{\widehat{\hat{i}} \hat{j}} J_{\underline{\hat{i}} \hat{j}}, \\
& \left\{Q_{ \pm}^{(-)}, Q_{\mp}^{(-)}\right\}=-2 \mathcal{C} \Gamma_{+} \mathcal{P}_{\mp} P_{-}-\sqrt{2} \lambda \mathcal{C} \widehat{\Gamma}^{\overline{\hat{i}} \hat{j}} J_{\overline{\hat{i}} \underline{\hat{j}}}, \\
& \left\{Q_{+}^{( \pm)}, Q_{+}^{(\mp)}\right\}=-2 \mathcal{C} \Gamma^{\overline{\hat{i}}} \ell_{\mp} \mathcal{P}_{+} P_{\hat{i}}-4 \lambda \mathcal{C} f \Gamma^{\underline{i}} \ell_{\mp} \mathcal{P}_{+} P_{\underline{i}}^{*} \mp 2 \lambda \mathcal{C} f \Gamma^{\underline{i^{\prime}}} \ell_{\mp} \mathcal{P}_{+} P_{\underline{i}^{\prime}}^{*}, \\
& \left\{Q_{+}^{( \pm)}, Q_{-}^{(\mp)}\right\}=-2 \mathcal{C} \Gamma^{\hat{i}} \ell_{\mp} \mathcal{P}_{-} P_{\hat{i}}-4 \lambda \mathcal{C} f \Gamma^{\bar{i}} \ell_{\mp} \mathcal{P}_{-} P_{\bar{i}}^{*} \mp 2 \lambda \mathcal{C} f \Gamma^{\bar{i}^{\prime}} \ell_{\mp} \mathcal{P}_{-} P_{\bar{i}^{\prime}}^{*} .
\end{aligned}
$$

Summarizing we have derived the NH superalgebra of a Euclidean M pp-wave brane as (7.18), (77.7) and (7.22).

We note that there exists a super-subalgebra of the NH superalgebra generated by $P_{\hat{i}}$, $P_{\underline{\hat{i}}}^{*}, J_{\overline{\hat{i}} \overline{\hat{j}}}, J_{\underline{\hat{i} \hat{j}}}$ and $Q_{+}^{( \pm)}$

$$
\begin{aligned}
& {\left[P_{\hat{i}}, J_{\overline{\hat{j}} \hat{\hat{k}}}\right]=\eta_{\eta_{\bar{i} \hat{j}}} P_{\hat{\hat{k}}}-\eta_{\overline{\hat{i}} \overline{\hat{k}}} P_{\hat{\bar{j}}}, \quad\left[P_{\underline{\hat{i}}}^{*}, J_{\underline{\hat{j}} \underline{\hat{k}}}\right]=\eta_{\underline{\hat{i}} \underline{\hat{j}}} P_{\underline{\hat{k}}}^{*}-\eta_{\underline{i} \underline{\hat{k}}} P_{\underline{\hat{j}}}^{*},}
\end{aligned}
$$

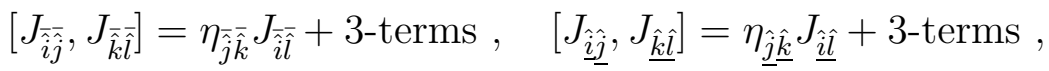

$$
\begin{aligned}
& {\left[P_{\bar{i}}, Q_{+}^{(-)}\right]=-\frac{\lambda}{\sqrt{2}} Q_{+}^{(+)} f \Gamma_{\bar{i}} \Gamma_{+}, \quad\left[P_{\bar{i}^{\prime}}, Q_{+}^{(-)}\right]=-\frac{\lambda}{2 \sqrt{2}} Q_{+}^{(+)} f \Gamma_{\bar{i}^{\prime}} \Gamma_{+},} \\
& {\left[J_{\overline{\hat{i}} \bar{j}}, Q_{+}^{\bullet}\right]=\frac{1}{2} Q_{+}^{(\bullet)} \Gamma_{\overline{\hat{i}} \bar{j}}, \quad\left[J_{\underline{\hat{i} \hat{j}}}, Q_{+}^{\bullet}\right]=\frac{1}{2} Q_{+}^{(\bullet)} \Gamma_{\underline{\hat{i}} \underline{j}}, \quad\left[P_{\underline{\hat{i}}}^{*}, Q_{+}^{(-)}\right]=\frac{1}{2 \sqrt{2}} Q_{+}^{(+)} \Gamma_{\underline{\hat{i}}} \Gamma_{+},} \\
& \left\{Q_{+}^{(-)}, Q_{+}^{(-)}\right\}=-\frac{\lambda}{\sqrt{2}} \mathcal{C} \widehat{\Gamma}^{\overline{\hat{i}} \overline{\hat{j}}} J_{\overline{\hat{i}} \overline{\hat{j}}}-\frac{\lambda}{\sqrt{2}} \mathcal{C} \widehat{\Gamma}^{\hat{i} \hat{j}}-J_{\underline{\underline{\hat{i}}} \hat{j}}, \\
& \left\{Q_{+}^{( \pm)}, Q_{+}^{(\mp)}\right\}=-2 \mathcal{C} \Gamma^{\overline{\hat{i}}} \ell_{\mp} \mathcal{P}_{+} P_{\hat{i}}-4 \lambda \mathcal{C} f \Gamma^{\underline{i}} \ell_{\mp} \mathcal{P}_{+} P_{\underline{i}}^{*} \mp 2 \lambda \mathcal{C} f \Gamma^{\underline{i}^{\prime}} \ell_{\mp} \mathcal{P}_{+} P_{\underline{i}^{\prime}}^{*} .
\end{aligned}
$$

This is the supersymmetrization of the Poincaré algebra generated by $\left\{P_{\hat{\hat{i}}}, J_{\overline{\hat{i}} \overline{\hat{j}}}\right\}$ which is the isometry on the brane worldvolume and the Lorentz symmetry in the transverse space generated by $\left\{P_{\underline{\hat{i}}}^{*}, J_{\underline{\hat{i}} \hat{j}}\right\}$. The conditions (17.10) and (17.11) ensure the existence of this super-subalgebra. 


\section{Branes in $\mathbf{A d S}_{4 / 7} \times \mathbf{S}^{7 / 4}$}

The action for an M2-brane [48] is composed of the NG action and the WZ action

$$
S=T \int_{\Sigma}\left[\mathcal{L}_{\mathrm{NG}}+\mathcal{L}_{\mathrm{WZ}}\right], \quad \mathcal{L}_{\mathrm{NG}}=d^{p+1} \xi \sqrt{s \operatorname{det} g_{i j}}
$$

where $s=-1$ for a Lorentzian brane and $s=1$ for a Euclidean brane. $T$ is the tension of the brane. For an M5-brane case, the self-duality of the two-form gauge field $B$ on the brane is imposed on the field equations, or the NG action is replaced by the PST action $[49]$

$$
\begin{aligned}
\mathcal{L}_{\mathrm{PST}} & =\sqrt{s \operatorname{det}\left(g_{i j}-i \alpha^{2} \mathcal{H}_{i j}^{*}\right)}+\alpha^{2} \frac{\sqrt{s \operatorname{det} g}}{4} \mathcal{H}^{* i j} \mathcal{H}_{i j}, \\
\mathcal{H}_{i j} & =\mathcal{H}_{i j k} v^{k}, \quad \mathcal{H}^{* i j}=\mathcal{H}^{* i j k} v_{k}, \quad v_{i}=\frac{\partial_{i} a}{\sqrt{g^{j k} \partial_{j} a \partial_{k} a}}, \\
\mathcal{H} & =H+\mathcal{C}_{3}, \quad \mathcal{H}^{* i j k}=\frac{1}{3 ! \sqrt{s \operatorname{det} g}} \epsilon^{i j k l m n} \mathcal{H}_{l m n}, \quad H=d B
\end{aligned}
$$

where $\mathcal{C}_{3}$ is a pullback of the three-form gauge field, and $\alpha^{2}=i \sqrt{s}$. Here the PST scalar field $a$ is contained in the M5-brane case as a modification of the usual DBI action. The WZ term is known to be characterized by manifestly supersymmetric $(p+2)$-form $h_{p+2}=d \mathcal{L}_{\mathrm{WZ}}$, which is composed of the pullback of the supercurrents, $\mathbf{L}^{A}$ and $L^{\alpha}$, on the supergroup manifold and the modified field strength $\mathcal{H}$. The $(p+2)$-form $h_{p+2}$ is closed but not exact on the superspace, because $\mathcal{L}_{\mathrm{WZ}}$ is not superinvariant but quasi-superinvariant. Expanding $h_{p+2}$ with respect to $\mathcal{H}$

$$
h_{p+2}\left(\mathbf{L}^{A}, L^{\alpha}, \mathcal{H}\right)=h^{(p+2)}\left(\mathbf{L}^{A}, L^{\alpha}\right)-\frac{c}{2} \mathcal{H} h^{(p-1)}\left(\mathbf{L}^{A}, L^{\alpha}\right)
$$

where $c$ is a constant determined below, the closedness condition $d h_{p+2}=0$ is expressed as

$$
\begin{aligned}
d h^{(p-1)} & =0, \\
d h^{(p+2)}-\frac{c}{2} d \mathcal{H} h^{(p-1)} & =0 .
\end{aligned}
$$

\subsection{CE-cohomology classification}

We show that $\mathrm{M} p$-brane actions in $\operatorname{AdS}_{q+2} \times \mathrm{S}^{9-q}(q=2,5)$ can be classified as non-trivial elements of the CE-cohomology on the differential algebra, MC equations (6.5) for the super-AdS $\mathrm{S}_{q+2} \times \mathrm{S}^{9-q}$ algebra. 
In order to avoid an additional dimensionful parameter, we assign dimensions as

$$
\begin{aligned}
& \begin{array}{llll}
\mathbf{L}^{A} & L^{\alpha} & \lambda \mathcal{H}
\end{array} \\
& \operatorname{dim} 11 / 2-13
\end{aligned}
$$

For structureless branes, the dimension of $S_{\mathrm{WZ}}$ must be equal to the dimension of $S_{\mathrm{NG}}$, from which we find $\operatorname{dim} h_{p+2}=p+1$ because $\operatorname{dim} h_{p+2}=\operatorname{dim} \mathcal{L}_{\mathrm{WZ}}=\operatorname{dim} \mathcal{L}_{\mathrm{NG}}=p+1$, and thus $\operatorname{dim} h^{(k)}=k-1$. $h^{(k)}$ is composed of $\mathbf{L}^{A}, L^{\alpha}$ and $\lambda$, and thus we can write $h^{(k)}$ as $\lambda^{l}\left(\mathbf{L}^{A}\right)^{m}\left(L^{\alpha}\right)^{n}$. The integers $l, m$ and $n$ are restricted by the properties of $h^{(k)}$, $\operatorname{dim} h^{(k)}=k-1$ and $\operatorname{deg} h^{(k)}=k$, as

$$
-l+m+\frac{1}{2} n=k-1, \quad m+n=k .
$$

For consistent flat limit, we demand $l \geq 0$ because $\lambda$ is related to the inverse of the radii of $A d S_{q+2}$ and $S^{9-q}$. In addition, we require that $\epsilon_{a_{1} \cdots a_{4}}$ and $\epsilon_{a_{1}^{\prime} \cdots a_{7}^{\prime}}$ are accompanied with $\lambda ; \lambda \epsilon_{a_{1} \cdots a_{4}}$ and $\lambda \epsilon_{a_{1}^{\prime} \cdots a_{7}^{\prime}}$, because $\epsilon_{a_{1} \cdots a_{4}}$ and $\epsilon_{a_{1}^{\prime} \cdots a_{7}^{\prime}}$ disappear in the flat limit. Noting that (8.7) implies $l=1-\frac{1}{2} n \leq 1$, we consider the cases with $l=0$ and 1 . It is easy to see that $(m, n)=(k-2,2)$ for $l=0$ while $(m, n)=(k, 0)$ for $l=1$. In the former case, terms of the form $\mathbf{L}^{A_{1}} \cdots \mathbf{L}^{A_{k-2}} \bar{L} \Gamma_{A_{1} \cdots A_{k-2}} L$ are candidates for $h^{(k)}$. These terms are non-trivial only for $k=3,4 \bmod 4$, because $C \Gamma_{A_{1} \cdots A_{k-2}}$ is symmetric if $k-2=1,2 \bmod 4$. In the latter case, $\lambda \epsilon_{a_{1} \cdots a_{4}} \mathbf{L}^{a_{1}} \cdots \mathbf{L}^{a_{4}}$ and $\lambda \epsilon_{a_{1}^{\prime} \cdots a_{7}^{\prime}} \mathbf{L}^{a_{1}^{\prime}} \cdots \mathbf{L}^{a_{7}^{\prime}}$ are candidates for $h^{(4)}$ and $h^{(7)}$, respectively. We summarize the non-trivial candidates for $h^{(k)}$

$$
\begin{aligned}
h^{(3)}: & \mathbf{L}^{A} \bar{L} \Gamma_{A} L \\
h^{(4)}: & \mathbf{L}^{A} \mathbf{L}^{B} \bar{L} \Gamma_{A B} L, \quad \lambda \epsilon_{a_{1} \cdots a_{4}} \mathbf{L}^{a_{1}} \cdots \mathbf{L}^{a_{4}} \\
h^{(7)}: & \mathbf{L}^{A_{1}} \cdots \mathbf{L}^{A_{5}} \bar{L} \Gamma_{A_{1} \cdots A_{5}} L, \quad \lambda \epsilon_{a_{1}^{\prime} \cdots a_{7}^{\prime}} \mathbf{L}^{a_{1}^{\prime}} \cdots \mathbf{L}^{a_{7}^{\prime}} \\
h^{(8)}: & \mathbf{L}^{A_{1}} \cdots \mathbf{L}^{A_{6}} \bar{L} \Gamma_{A_{1} \cdots A_{6}} L
\end{aligned}
$$

where $\mathbf{L}^{A} \bar{L} \Gamma_{A} L$ stands for two candidates $\mathbf{L}^{a} \bar{L} \Gamma_{a} L$ and $\mathbf{L}^{a^{\prime}} \bar{L} \Gamma_{a^{\prime}} L$, and so on. For example, $h^{(4)}$ is of the form

$$
h^{(4)}=c_{1} \mathbf{L}^{a} \mathbf{L}^{b} \bar{L} \Gamma_{a b} L+c_{2} \mathbf{L}^{a} \mathbf{L}^{a^{\prime}} \bar{L} \Gamma_{a a^{\prime}} L+c_{3} \mathbf{L}^{a^{\prime}} \mathbf{L}^{b^{\prime}} \bar{L} \Gamma_{a^{\prime} b^{\prime}} L+c_{4} \lambda \epsilon_{a_{1} \cdots a_{4}} \mathbf{L}^{a_{1}} \cdots \mathbf{L}^{a_{4}} .
$$

Next we are going to find $h^{(k)}$ satisfying (8.4) and (8.5). The first step for this is to find a closed form $d h^{(k)}=0$ in (8.4). $h^{(k)}$ can be a closed form only when $k=4$. This is due to the Fierz identity

$$
\left(\mathcal{C} \Gamma_{A B}\right)_{(\alpha \beta}\left(\mathcal{C} \Gamma^{B}\right)_{\gamma \delta)}=0
$$


The coefficients are fixed by the closedness condition $d h^{(4)}=0$ as

$$
h^{(4)}=c\left[\frac{1}{2} \mathbf{L}^{A} \mathbf{L}^{B} \bar{L} \Gamma_{A B} L-\frac{6 \lambda}{4 !} \epsilon_{a_{1} \cdots a_{4}} \mathbf{L}^{a_{1}} \cdots \mathbf{L}^{a_{4}}\right]
$$

where $\epsilon_{0123}=-\epsilon_{\natural 123}=+1$. As seen in Appendix $\mathbb{B}$, the overall coefficient $c$ is fixed by the requirement of the $\kappa$-invariance $[48,50]$ of the total action $S$ as $c=-1$ for Lorentzian brane and $c=i$ for Euclidean brane: $c=i \sqrt{s}$. Using $h^{(4)}$ above, the closed four-form $h_{4}$ is constructed as

$$
h_{4}=h^{(4)} .
$$

Because $h_{4}$ is not exact on the superspace as will be shown below, we find that the M2-brane action in $\mathrm{AdS}_{4 / 7} \times \mathrm{S}^{7 / 4}$ is a non-trivial element of CE cohomology of the differential algebra (6.5), MC equations for super- $\mathrm{AdS}_{4 / 7} \times \mathrm{S}^{7 / 4}$ algebra. The obtained action is consistent with one given in [50].

Next we introduce $d \mathcal{H}$ to the differential algebra (6.5). Since $\mathcal{H}=d B+\mathcal{C}_{3}$ and $h_{4}=c^{\prime} d \mathcal{C}_{3}$ with a constant $c^{\prime}, d \mathcal{H}$ is given by

$$
c^{\prime} d \mathcal{H}=h_{4}=h^{(4)}
$$

where $h^{(4)}$ is given in (8.14). If we can construct $h^{(7)}$ satisfying (8.5), then $h_{7}$ turns out to be a closed seven-form. We find that using (8.14) and (8.16) the condition (8.5) fixes coefficients of a linear combination of candidates as

$$
h^{(7)}=c^{2}\left[\frac{1}{5 !} \mathbf{L}^{A_{1}} \cdots \mathbf{L}^{A_{5}} \bar{L} \Gamma_{A_{1} \cdots A_{5}} L-\frac{6 \lambda}{7 !} \epsilon_{a_{1}^{\prime} \cdots a_{7}^{\prime}} \mathbf{L}^{a_{1}^{\prime}} \cdots \mathbf{L}^{a_{7}^{\prime}}\right]
$$

where $\epsilon_{4 \ldots 9 \natural}=-\epsilon_{4 \ldots 90}=+1$ and $c^{\prime} \equiv-c$. To see this we have used the Fierz identity,

$$
\left(\mathcal{C} \Gamma^{A_{1} \cdots A_{5}}\right)_{(\alpha \beta}\left(\mathcal{C} \Gamma_{A_{5}}\right)_{\gamma \delta)}-3\left(\mathcal{C} \Gamma^{\left[A_{1} A_{2}\right.}\right)_{(\alpha \beta}\left(\mathcal{C} \Gamma^{\left.A_{3} A_{4}\right]}\right)_{\gamma \delta)}=0
$$

The closed seven-form is constructed using (8.14) and (8.17) as

$$
h_{7}=h^{(7)}-\frac{c}{2} h^{(4)} \mathcal{H} .
$$

Because $h_{7}$ is not exact on the superspace as will be shown below, we find that M5-brane action in $\mathrm{AdS}_{4 / 7} \times \mathrm{S}^{7 / 4}$ is characterized as a non-trivial element of CE cohomology on the differential algebra (6.5) and (8.16). The constant $c^{2}$ is determined by the requirement that the total action is $\kappa$-invariant $[49,51]$ as $c^{2}=-1$ and $i$ for Lorentzian and Euclidean 
branes respectively, i.e. $c^{2}=\alpha^{2}=i \sqrt{s}$. See Appendix B. The obtained action is consistent with one given in [51].

We show that the four- and seven-forms obtained above are not exact. Suppose that $h_{4}$ is exact, then there must exist $b^{(3)}$ such that $h^{(4)}=d b^{(3)} . b^{(3)}$ can be written as $\lambda^{l}\left(\mathbf{L}^{A}\right)^{m}\left(L^{\alpha}\right)^{n}$ where integers $l, m$ and $n$ are restricted by the properties of $b^{(3)}, \operatorname{dim} b^{(3)}=3$ and $\operatorname{deg} b^{(3)}=3$. This implies that $l \leq 0$. We find that there is no candidate for $l=0$. For $l=-1$, we find two candidates, $\lambda^{-1} \mathbf{L}^{a} \bar{L} \Gamma_{a} L$ and $\lambda^{-1} \mathbf{L}^{a^{\prime}} \bar{L} \Gamma_{a^{\prime}} L$, but any linear combination of them does not satisfy $h^{(4)}=d b^{(3)}$. It is obvious that terms with $l \leq-2$ do not satisfy $h^{(4)}=d b^{(3)}$. Thus, $h_{4}$ is not exact. Next, suppose that $h_{7}$ is exact, then there exists $b_{6}$ such that $h_{7}=d b_{6}$. This implies, expanding $b_{6}\left(\mathbf{L}^{\mu}, L^{\alpha}, \mathcal{H}\right)$ as $b^{(6)}\left(\mathbf{L}^{A}, L^{\alpha}\right)+\frac{1}{2} \mathcal{H} b^{(3)}\left(\mathbf{L}^{A}, L^{\alpha}\right)$, that

$$
h^{(7)}=d b^{(6)}-\frac{c}{2} d \mathcal{H} b^{(3)}, \quad h^{(4)}=-d b^{(3)} .
$$

Because we have shown that $h^{(4)}$ is not exact, there dose not exist $b^{(3)}$ satisfying (8.20). Thus, we have shown that $h_{7}$ is not exact.

Summarizing we find that actions of M2- and M5-branes in $\mathrm{AdS}_{4 / 7} \times \mathrm{S}^{7 / 4}$ are characterized as non-trivial elements of the CE cohomology.

\section{2 $(p+1)$-dimensional form of the WZ term}

We derive $(p+1)$-dimensional form of the WZ-term following [51].

The supervielbein and super spin connection are given in Appendix A.2. These satisfy the following differential equations

$$
\begin{aligned}
\partial_{t} \hat{\mathbf{L}}^{A} & =-2 \bar{\theta} \Gamma^{A} \hat{L}, \\
\partial_{t} \hat{\mathbf{L}}^{A B} & =2 \lambda \hat{\theta} \widehat{\Gamma}^{A B} \hat{L}, \\
\partial_{t} \hat{L} & =d \theta-\frac{\lambda}{2} \hat{\mathbf{L}}^{A} \widehat{\Gamma}_{A} \theta+\frac{1}{4} \hat{\mathbf{L}}^{A B} \Gamma_{A B} \theta,
\end{aligned}
$$

where the symbols with "hat" implies that the fermionic variable $\theta$ is rescaled as $\theta \rightarrow t \theta$.

By using these equations, one finds that

$$
\partial_{t} \hat{h}_{4}=d b_{3}, \quad b_{3}=-c \hat{\mathbf{L}}^{A} \hat{\mathbf{L}}{ }^{B} \hat{\bar{L}} \Gamma_{A B} \theta
$$

This implies that

$$
h_{4}=d \mathcal{C}_{3}, \quad \mathcal{C}_{3}=\int_{0}^{1} d t b_{3}+C^{(3)}
$$


where $C^{(3)}$ is a bosonic 3 -form satisfying $d C^{(3)}=\left.h_{4}\right|_{\text {bosonic }}$. It follows from

$$
-c \mathcal{H}=d B+\int_{0}^{1} d t b_{3}+C^{(3)}
$$

that

$$
-c \partial_{t} \hat{\mathcal{H}}=b_{3}
$$

In the similar way, one derives

$$
\partial_{t} \hat{h}_{7}=d\left(b_{6}-\frac{c}{2} b_{3} \hat{\mathcal{H}}\right), \quad b_{6}=c^{2} \frac{2}{5 !} \hat{\mathbf{L}}^{A_{1}} \cdots \hat{\mathbf{L}}^{A_{5}} \hat{\bar{L}} \Gamma_{A_{1} \cdots A_{5}} \theta
$$

so that

$$
h_{7}=d \mathcal{C}_{6}, \quad \mathcal{C}_{6}=\int_{0}^{1} d t\left(b_{6}-\frac{c}{2} b_{3} \hat{\mathcal{H}}\right)+C^{(6)}
$$

where $C^{(6)}$ is a bosonic 6 -form satisfying $d C^{(6)}=\left.h_{7}\right|_{\text {bosonic }}$.

Summarizing the $(p+1)$-dimensional form of the WZ term is given as

$$
\begin{aligned}
& \mathcal{L}_{\mathrm{WZ}}^{M 2}=-i \sqrt{s} \int_{0}^{1} d t \hat{\mathbf{L}}^{A} \hat{\mathbf{L}}^{B} \hat{\bar{L}} \Gamma_{A B} \theta+C^{(3)} \\
& \mathcal{L}_{\mathrm{WZ}}^{M 5}=i \sqrt{s} \int_{0}^{1} d t\left(\frac{2}{5 !} \hat{\mathbf{L}}^{A_{1}} \cdots \hat{\mathbf{L}}^{A_{5}} \hat{\bar{L}} \Gamma_{A_{1} \cdots A_{5}} \theta-\frac{1}{2} \hat{\mathbf{L}}^{A} \hat{\mathbf{L}}{ }^{B} \hat{\bar{L}} \Gamma_{A B} \theta \hat{\mathcal{H}}\right)+C^{(6)} .
\end{aligned}
$$

\section{Non-relativistic Branes in $\operatorname{AdS}_{4 / 7} \times \mathbf{S}^{7 / 4}$}

We consider the non-relativistic limit of the branes in $\mathrm{AdS}_{4 / 7} \times \mathrm{S}^{7 / 4}$ obtained in the previous section.

We scale coordinates and the tension as

$$
\begin{aligned}
& X^{\underline{A}} \rightarrow \Omega X^{\underline{A}}, \quad \theta_{-} \rightarrow \Omega \theta_{-}, \\
& T=T_{\mathrm{NR}} \Omega^{-2}, \quad H=\Omega H_{1} .
\end{aligned}
$$

(9.1) is consistent with (6.3). As can be seen from the concrete expression of the supercurrents given in Appendix A.2, the supercurrents are expanded as (5.3). Expanding $\mathbf{L}^{A B}$ as in (5.4) and substituting (5.3) and (5.4) into the MC equation (6.5), one finds that Cartan one forms $\left\{\mathbf{L}_{m}^{\bar{A}}, \mathbf{L} \frac{A}{m}, \mathbf{L}_{m}^{\bar{A} \bar{B}}, \mathbf{L}_{m}^{\bar{A}} \underline{B}, \mathbf{L} \frac{A B}{m}, L_{ \pm m} \mid m=0,1\right\}$ form the MC equation (6.14) $-(6.22)$. 


\subsection{M2-brane}

First we consider an M2-brane. The NG part $\mathcal{L}_{\mathrm{NG}}$ is expanded as in (5.15) with (5.16) and (5.17). By using (6.14) and $L_{+}=M L_{+}$with (6.6), one derives

$$
d \mathcal{L}_{\mathrm{NG}}^{\operatorname{div}}=d\left(\operatorname{det}\left(\mathbf{L}_{0}^{\bar{A}}\right)_{i} d^{3} \xi\right)=-\frac{i \sqrt{s}}{2 !} \mathbf{L}_{0}^{\bar{A}_{1}} \mathbf{L}_{0}^{\bar{A}_{2}} \bar{L}_{+0} \Gamma_{\bar{A}_{1} \bar{A}_{2}} L_{+0}
$$

Since

$$
M^{\prime} \Gamma^{\bar{A} \bar{B}}=\Gamma^{\bar{A} \bar{B}} M
$$

the four-form $h_{4}$ is expanded as

$$
T h_{4}=T_{\mathrm{NR}} \Omega^{-2} h_{4}^{d i v}+T_{\mathrm{NR}} h_{4}^{\text {fin }}+O\left(\Omega^{4}\right)
$$

with

$$
\begin{aligned}
h_{4}^{d i v}= & \frac{i \sqrt{s}}{2} \mathbf{L}_{0}^{\bar{A}} \mathbf{L}_{0}^{\bar{B}} \bar{L}_{+0} \Gamma_{\bar{A} \bar{B}} L_{+0}, \\
h_{4}^{f i n}= & \frac{i \sqrt{s}}{2}\left[\mathbf{L}_{0}^{\bar{A}} \mathbf{L}_{0}^{\bar{B}} \bar{L}_{-1} \Gamma_{\bar{A} \bar{B}} L_{-1}+2 \mathbf{L}_{0}^{\bar{A}} \mathbf{L}_{0}^{\bar{B}} \bar{L}_{+0} \Gamma_{\bar{A} \bar{B}} L_{+2}+2 \mathbf{L}_{0}^{\bar{A}} \mathbf{L}_{2}^{\bar{B}} \bar{L}_{+0} \Gamma_{\bar{A} \bar{B}} L_{+0}\right. \\
& +4 \mathbf{L}_{0}^{\bar{A}} \mathbf{L}_{1}^{\underline{B}} \bar{L}_{+0} \Gamma_{\bar{A}} \underline{B}_{-1} L_{-1}+\mathbf{L}_{1}^{\frac{A}{1}} \mathbf{L}_{1}^{\underline{B}} \bar{L}_{+0} \Gamma_{\underline{A B}} L_{+0} \\
& \left.-6 \lambda \delta^{(2,1)} \epsilon_{\bar{a}_{1} \bar{a}_{2} \underline{a}_{3} \underline{a}_{4}} e_{0}^{\bar{a}_{1}} e_{0}^{\bar{a}_{2}} e_{1}^{\underline{a}_{3}} e_{1}^{\underline{a_{4}}}\right] .
\end{aligned}
$$

This implies that the bosonic 3 -form $C^{3}$ is expanded as

$$
\begin{aligned}
T d C^{(3)} & =T_{\mathrm{NR}} \Omega^{-2} d C_{0}^{(3)}+T_{\mathrm{NR}} d C_{2}^{(3)}+O\left(\Omega^{2}\right), \\
d C_{0}^{(3)} & =0 \\
d C_{2}^{(3)} & =-3 i \sqrt{s} \lambda \delta^{(2,1)} \epsilon_{\bar{a}_{1} \bar{a}_{2} \underline{a}_{3} \underline{a}_{4}} e_{0}^{\bar{a}_{1}} e_{0}^{\bar{a}_{2}} e_{1}^{\underline{a}_{3}} e_{1}^{\underline{a}_{4}} .
\end{aligned}
$$

Since

$$
d\left(\operatorname{det}\left(\mathbf{L}_{0}^{\bar{A}}\right)_{i} d^{3} \xi\right)+h_{4}^{d i v}=0
$$

the fermionic contribution of $\mathcal{L}_{\mathrm{NG}}^{d i v}$ and $\mathcal{L}_{\mathrm{WZ}}^{\text {div }}$ cancel each other. In order to delete the bosonic terms of $\mathcal{L}_{\mathrm{NG}}^{\text {div }}+\mathcal{L}_{\mathrm{WZ}}^{\text {div }}$, we choose

$$
C_{0}^{(3)}=-\frac{1}{3 !} \epsilon_{\bar{A}_{0} \bar{A}_{1} \bar{A}_{2}} e_{0}^{\bar{A}_{0}} e_{0}^{\bar{A}_{1}} e_{0}^{\bar{A}_{2}}
$$

It follows from the expressions given in Appendix A.2 that $d C_{0}^{(3)}=0$. In summary, we find that the gluing matrix $M$ leads to the consistent non-relativistic limit of the M2-brane in $\mathrm{AdS}_{4 / 7} \times \mathrm{S}^{7 / 4}$. 
The non-relativistic M2-brane action is given as

$$
S_{\mathrm{NR}}=T_{\mathrm{NR}} \int_{\Sigma}\left[\mathcal{L}_{\mathrm{NG}}^{f i n}+\mathcal{L}_{\mathrm{WZ}}^{f i n}\right]
$$

with (5.17) and

$$
\begin{aligned}
\mathcal{L}_{\mathrm{WZ}}^{f i n}= & -i \sqrt{s} \int_{0}^{1} d t\left[\hat{\mathbf{L}}_{0}^{\bar{A}} \hat{\mathbf{L}}_{0}^{\bar{B}}\left(\hat{\bar{L}}_{-1} \Gamma_{\bar{A} \bar{B}} \theta_{-}+\hat{\bar{L}}_{+2} \Gamma_{\bar{A} \bar{B}} \theta_{+}\right)+2 \hat{\mathbf{L}}_{0}^{\bar{A}} \hat{\mathbf{L}}_{2}^{\bar{B}} \hat{\bar{L}}_{+0} \Gamma_{\bar{A} \bar{B}} \theta_{+}\right. \\
& \left.+2 \hat{\mathbf{L}}_{0}^{\bar{A}} \hat{\mathbf{L}}_{1}^{\underline{B}}\left(\hat{\bar{L}}_{-1} \Gamma_{\bar{A}} \theta_{+}+\hat{\bar{L}}_{+0} \Gamma_{\bar{A}} \theta_{-} \theta_{-}\right)+\hat{\mathbf{L}} \frac{A}{1} \hat{\mathbf{L}} \frac{B}{1} \hat{\bar{L}}_{+0} \Gamma_{\underline{A B}} \theta_{+}\right]+C_{2}^{(3)}
\end{aligned}
$$

The bosonic contribution is

$$
\begin{aligned}
\int_{\Sigma} C_{2}^{(3)} & =-3 i \sqrt{s} \lambda \delta^{(2,1)} \int_{\Sigma} \operatorname{vol}_{\Sigma_{2}} \epsilon_{a \underline{a b}} y^{\underline{a}} d y^{\underline{b}} \\
& =-3 i \sqrt{s} \lambda \delta^{(2,1)} \int d^{3} \xi \sqrt{s \operatorname{det} g_{0}} \epsilon_{\underline{a b}} y^{\underline{a}} \partial_{i^{\prime}} y^{\underline{\underline{b}}}
\end{aligned}
$$

where $i^{\prime}$ represent worldvolume directions in $\mathrm{S}^{7}$ or $\mathrm{AdS}_{7}$.

The $t$-integration is easily done after fixing the $\kappa$-gauge symmetry by $\theta_{+}=0$ (see Appendix [B], which leads to

$$
\begin{aligned}
& \mathbf{L}_{0}^{\bar{A}}=e_{0}^{\bar{A}}, \quad \mathbf{L}_{2}^{\bar{A}}=e_{2}^{\bar{A}}-\bar{\theta}_{-} \Gamma^{\bar{A}} \mathrm{D} \theta_{-}, \quad \mathbf{L}_{1}^{\frac{A}{1}}=e_{1}^{\frac{A}{1}} \\
& L_{-1}=\mathrm{D} \theta_{-}=d \theta_{-}-\frac{\lambda}{2} e_{0}^{\bar{A}} \widehat{\Gamma}_{\bar{A}} \theta_{-}+\frac{1}{4} \omega^{\bar{A} \bar{B}} \Gamma_{\bar{A} \bar{B}} \theta_{-} \\
& \left(g_{0}\right)_{i j}=\left(e_{0}^{\bar{A}}\right)_{i}\left(e_{0}^{\bar{B}}\right)_{j} \eta_{\bar{A} \bar{B}} .
\end{aligned}
$$

$\left(e_{0}^{\bar{A}}\right)_{i}$ is the vielbein on the worldvolume in the static gauge, $x^{\bar{A}}=\xi^{i}$. Substituting these into the non-relativistic action we obtain

$$
\begin{aligned}
S_{\mathrm{NR}}= & T_{\mathrm{NR}} \int d^{3} \xi \sqrt{s \operatorname{det} g_{0}}\left[g_{0}^{i j} \partial_{i} y^{\underline{A}} \partial_{i} y^{\underline{B}} \eta_{\underline{A B}}+\frac{\epsilon^{2} \lambda^{2}}{2}\left(4 m y^{2}-n y^{\prime 2}\right)-2 \bar{\theta}_{-} \gamma^{i} \mathrm{D}_{i} \theta_{-}\right] \\
& +T_{\mathrm{NR}} \int_{\Sigma} C_{2}^{(3)} .
\end{aligned}
$$

In the flat limit $\lambda \rightarrow 0$, this reproduces the non-relativistic action given in [11].

\section{$9.2 \quad$ M5-brane}

Next we consider an M5-brane for which $c^{2}=i \sqrt{s}$. In this case the gluing matrix

$$
M=\sqrt{-s} \Gamma^{\bar{A}_{0} \cdots \bar{A}_{5}}, \quad M^{\prime}=-M
$$

satisfies

$$
M^{\prime} \Gamma_{\bar{B}_{1} \bar{B}_{2}}=-\Gamma_{\bar{B}_{1} \bar{B}_{2}} M
$$


so that $\mathcal{H}$ is of order $\Omega$

$$
\begin{aligned}
\mathcal{H} & =\Omega \mathcal{H}_{1}+O\left(\Omega^{3}\right) \\
\mathcal{H}_{1} & =H_{1}+\int_{0}^{1} d t\left[\hat{\mathbf{L}}_{0}^{\bar{A}} \hat{\mathbf{L}}_{0}^{\bar{B}}\left(\hat{\bar{L}}_{+0} \Gamma_{\bar{A} \bar{B}} \theta_{-}+\hat{\bar{L}}_{-1} \Gamma_{\bar{A} \bar{B}} \theta_{+}\right)+2 \hat{\mathbf{L}}_{0}^{\bar{A}} \hat{\mathbf{L}}_{1}^{\underline{B}} \hat{\bar{L}}_{+0} \Gamma_{\bar{A}}{ }_{B} \theta_{+}\right] .
\end{aligned}
$$

The PST part $\mathcal{L}_{\mathrm{PST}}$ is expanded as

$$
T \mathcal{L}_{\mathrm{PST}}=T_{\mathrm{NR}} \Omega^{-2} \mathcal{L}_{\mathrm{PST}}^{d i v}+T_{\mathrm{NR}} \mathcal{L}_{\mathrm{PST}}^{f i n}+O\left(\Omega^{4}\right)
$$

with

$$
\begin{aligned}
& \mathcal{L}_{\mathrm{PST}}^{d i v}=\sqrt{s \operatorname{det} g_{0}} d^{6} \xi \\
& \mathcal{L}_{\mathrm{PST}}^{f i n}=\sqrt{s \operatorname{det} g_{0}} d^{6}\left[\frac{1}{2} g_{0}^{i j}\left(g_{2}\right)_{i j}+\frac{1}{2}\left(\mathcal{H}_{1}^{-}\right)_{i j}\left(\mathcal{H}_{1}^{*}\right)^{i j}\right]
\end{aligned}
$$

where $g_{0}$ and $g_{2}$ are given in (5.18) and (5.19), and $\mathcal{H}_{1}^{-}$is defined as

$$
\mathcal{H}_{1}^{-}=\frac{c^{2}}{2}\left(\mathcal{H}_{1}+c^{2} \mathcal{H}_{1}^{*}\right)
$$

Noting that

$$
M^{\prime} \Gamma_{\bar{B}_{1} \cdots \bar{B}_{5}}=\Gamma_{\bar{B}_{1} \cdots \bar{B}_{5}} M
$$

$h_{7}$ is expanded as

$$
T h_{7}=T_{\mathrm{NR}} \Omega^{-2} h_{7}^{d i v}+T_{\mathrm{NR}} h_{7}^{f i n}+O\left(\Omega^{3}\right)
$$

with

$$
h_{7}^{d i v}=i \sqrt{s} \frac{1}{5 !} \mathbf{L}_{0}^{\bar{A}_{1}} \cdots \mathbf{L}_{0}^{\bar{A}_{5}} \bar{L}_{+0} \Gamma_{\bar{A}_{1} \cdots \bar{A}_{5}} L_{+0}
$$

and

$$
\begin{aligned}
& h_{7}^{f i n}=h_{2}^{(7)}-\frac{c}{2} \mathcal{H}_{1} h_{1}^{(4)}, \\
& h_{2}^{(7)}=\frac{i \sqrt{s}}{5 !}\left[\mathbf{L}_{0}^{\bar{A}_{1}} \cdots \mathbf{L}_{0}^{\bar{A}_{5}} \bar{L}_{-1} \Gamma_{\bar{A}_{1} \cdots \bar{A}_{5}} L_{-1}+2 \mathbf{L}_{0}^{\bar{A}_{1}} \cdots \mathbf{L}_{0}^{\bar{A}_{5}} \bar{L}_{+0} \Gamma_{\bar{A}_{1} \cdots \bar{A}_{5}} L_{+2}\right. \\
& +10 \mathbf{L}_{0}^{\bar{A}_{1}} \cdots \mathbf{L}_{0}^{\bar{A}_{4}} \mathbf{L}_{1}^{A_{5}} \bar{L}_{+0} \Gamma_{\bar{A}_{1} \cdots \bar{A}_{4} \underline{A}_{5}} L_{-1}+5 \mathbf{L}_{0}^{\bar{A}_{1}} \cdots \mathbf{L}_{0}^{\bar{A}_{4}} \mathbf{L}_{2}^{\bar{A}_{5}} \bar{L}_{+0} \Gamma_{\bar{A}_{1} \cdots \bar{A}_{5}} L_{+0}
\end{aligned}
$$

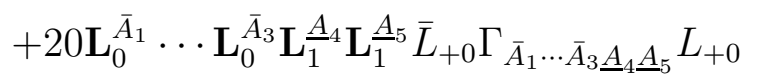

$$
\begin{aligned}
& \left.-\delta^{(1,5)} 6 \lambda \epsilon_{\bar{a}_{1}^{\prime} \cdots \bar{a}_{5}^{\prime} \underline{a}_{6}^{\prime} \underline{a}_{7}^{\prime}} \mathbf{L}_{0}^{\bar{a}_{1}^{\prime}} \cdots \mathbf{L}_{0}^{\bar{a}_{5}^{\prime}} \mathbf{L}_{1}^{\underline{a}_{6}^{\prime}} \mathbf{L}_{1}^{\underline{a}_{7}^{\prime}}\right] \text {, } \\
& h_{1}^{(4)}=c\left[\mathbf{L}_{0}^{\bar{A}} \mathbf{L}_{0}^{\bar{B}} \bar{L}_{+0} \Gamma_{\bar{A} \bar{B}} L_{-1}+\mathbf{L}_{0}^{\bar{A}} \mathbf{L}_{1}^{\underline{B}} \bar{L}_{+0} \Gamma_{\bar{A}}{ }_{\underline{B}} L_{+0}\right. \\
& \left.-\delta^{(3,3)} \frac{6 \lambda}{3 !} \epsilon_{\bar{a}_{1} \bar{a}_{2} \bar{a}_{3} \underline{a}_{4}} \mathbf{L}_{0}^{\bar{a}_{1}} \mathbf{L}_{0}^{\bar{a}_{2}} \mathbf{L}_{0}^{\bar{a}_{3}} \mathbf{L}_{1}^{\underline{a}_{4}}\right] \text {. }
\end{aligned}
$$


This implies that the bosonic 6-form $C^{(6)}$ is expanded as

$$
\begin{aligned}
T d C^{(6)} & =T_{\mathrm{NR}} \Omega^{-2} d C_{0}^{(6)}+T_{\mathrm{NR}} d C_{2}^{(6)}+O\left(\Omega^{2}\right) \\
d C_{0}^{(6)} & =0 \\
d C_{2}^{(6)} & =-\frac{6 c^{2}}{5 !} \delta^{(1,5)} \epsilon_{\bar{a}_{1}^{\prime} \cdots \bar{a}_{5}^{\prime} \underline{a}_{6}^{\prime} \underline{a}_{7}^{\prime}} e_{0}^{\bar{a}_{1}^{\prime}} \cdots e_{0}^{\bar{a}_{5}^{\prime}} e_{1}^{\underline{a}_{6}^{\prime}} e_{1}^{\underline{a}_{7}^{\prime}}+\frac{c^{2}}{2} \lambda \delta^{(3,3)} \epsilon_{\bar{a}_{1} \cdots \bar{a}_{3} \underline{a}_{4}} e_{0}^{\bar{a}_{1}} \cdots e_{0}^{\bar{a}_{3}} e_{1}^{\underline{a}_{4}} H_{1} \cdot
\end{aligned}
$$

Since

$$
d \mathcal{L}_{\mathrm{PST}}^{d i v}=d\left(\operatorname{det}\left(\mathbf{L}_{0}^{\bar{A}}\right)_{i} d^{6} \xi\right)=-h_{7}^{d i v}
$$

the fermionic contribution of $\mathcal{L}_{\mathrm{PST}}^{d i v}+\mathcal{L}_{\mathrm{WZ}}^{d i v}$ cancels out. The bosonic terms of $\mathcal{L}_{\mathrm{PST}}^{\text {div }}+\mathcal{L}_{\mathrm{WZ}}^{d i v}$, $\frac{1}{6 !} \epsilon_{\bar{A}_{0} \cdots \bar{A}_{5}} e_{0}^{\bar{A}_{0}} \cdots e_{0}^{\bar{A}_{5}}+C_{0}^{(6)}$, are deleted by choosing

$$
C_{0}^{(6)}=-\frac{1}{6 !} \epsilon_{\bar{A}_{0} \cdots \bar{A}_{5}} e_{0}^{\bar{A}_{0}} \cdots e_{0}^{\bar{A}_{5}}
$$

which satisfies $d C_{0}^{(6)}=0$. In summary, we find that the gluing matrix $M$ leads to the consistent non-relativistic limit of the M5-brane in $\mathrm{AdS}_{4 / 7} \times \mathrm{S}^{7 / 4}$.

The non-relativistic M5-brane action is composed of $\mathcal{L}_{\mathrm{PST}}^{\text {fin }}$ in (9.22) and

$$
\begin{aligned}
\mathcal{L}_{\mathrm{WZ}}^{f i n}= & c^{2} \int_{0}^{1} d t\left[\left(\frac{2}{5 !} \hat{\mathbf{L}}_{0}^{\bar{A}_{1}} \cdots \hat{\mathbf{L}}_{0}^{\bar{A}_{5}}\left(\hat{\bar{L}}_{-1} \Gamma_{\bar{A}_{1} \cdots \bar{A}_{5}} \theta_{-}+\hat{\bar{L}}_{+2} \Gamma_{\bar{A}_{1} \cdots \bar{A}_{5}} \theta_{+}\right)\right.\right. \\
& +\frac{2}{4 !} \hat{\mathbf{L}}_{0}^{\bar{A}_{1}} \cdots \hat{\mathbf{L}}_{0}^{\bar{A}_{4}} \hat{\mathbf{L}}_{1}^{\underline{A}_{5}}\left(\hat{\bar{L}}_{-1} \Gamma_{\bar{A}_{1} \cdots \bar{A}_{4} \underline{A}_{5}} \theta_{+}+\hat{\bar{L}}_{+0} \Gamma_{\bar{A}_{1} \cdots \bar{A}_{4} \underline{A}_{5}} \theta_{-}\right) \\
& +\frac{2}{3 !} \hat{\mathbf{L}}_{0}^{\bar{A}_{1}} \cdots \hat{\mathbf{L}}_{0}^{\bar{A}_{3}} \hat{\mathbf{L}}_{1}^{\underline{A}_{4}} \hat{\mathbf{L}}_{1}^{\underline{A}_{5}} \hat{\bar{L}}_{+0} \Gamma_{\bar{A}_{1} \cdots \bar{A}_{3} \underline{A}_{4} \underline{A}_{5} \theta_{+}} \\
& \left.+\frac{2}{4 !} \hat{\mathbf{L}}_{0}^{\bar{A}_{1}} \cdots \hat{\mathbf{L}}_{0}^{\bar{A}_{4}} \hat{\mathbf{L}}_{2}^{\bar{A}_{5}} \hat{\bar{L}}_{+0} \Gamma_{\bar{A}_{1} \cdots \bar{A}_{5}} \theta_{+}\right) \\
& \left.+\frac{1}{2}\left(\hat{\mathbf{L}}_{0}^{\bar{A}} \hat{\mathbf{L}}_{0}^{\bar{B}}\left(\hat{\bar{L}}_{+0} \Gamma_{\bar{A} \bar{B}} \theta_{-}+\hat{\bar{L}}_{-1} \Gamma_{\bar{A} \bar{B}} \theta_{+}\right)+2 \hat{\mathbf{L}}_{0}^{\bar{A}} \hat{\mathbf{L}}_{1}^{B} \hat{\bar{L}}_{+0} \Gamma_{\bar{A} B} \theta_{+}\right) \hat{\mathcal{H}}_{1}\right] \\
& +C_{2}^{(6)} .
\end{aligned}
$$

The bosonic contribution is

$$
\begin{aligned}
\int_{\Sigma} C_{2}^{(6)} & =6 c^{2} \lambda \delta^{(1,5)} \int_{\Sigma} \operatorname{vol}_{\Sigma_{5}^{\prime}} \epsilon_{\underline{a}^{\prime} \underline{b}^{\prime}} y^{\underline{a}^{\prime}} d y^{{\underline{b^{\prime}}}^{\prime}}+3 c^{2} \lambda \delta^{(3,3)} \int_{\Sigma} \operatorname{vol}_{\Sigma_{3}} y H_{1} \\
& =3 i \sqrt{s} \lambda \int d^{6} \xi \sqrt{s \operatorname{det} g_{0}}\left[2 \delta^{(1,5)} \epsilon_{\underline{a}^{\prime} \underline{b}^{\prime}} y^{\underline{a^{\prime}}} \partial_{\xi} y^{\underline{\underline{b}^{\prime}}}-\delta^{(3,3)} \partial_{i^{\prime}} y\left({ }^{*} B_{1}\right)^{i^{\prime}}\right]
\end{aligned}
$$

where $\xi$ and $i^{\prime}$ represent coordinates on $\Sigma_{1}$ and $\Sigma_{3}^{\prime}$ respectively, and $y$ is the transverse direction in $\mathrm{AdS}_{4}$ or $\mathrm{S}^{4}$. * means the Hodge dual in $\Sigma_{3}^{\prime}$. 
Let us fix the $\kappa$-symmetry by $\theta_{+}=0$. The $\theta$-dependent term in $\mathcal{H}$ disappears and so we have $\mathcal{H}_{1}=H_{1}$ in this gauge. The $t$-integration is easily done, and the action is drastically simplified as

$$
\begin{aligned}
\mathcal{L}_{\mathrm{PST}}^{f i n}= & d^{6} \xi \sqrt{s \operatorname{det} g_{0}}\left[-\bar{\theta}_{-} \gamma^{i} \mathrm{D}_{i} \theta_{-}+\frac{1}{2} g_{0}^{i j} \partial_{i} y^{\underline{A}} \partial_{j} y^{\underline{B}} \eta_{\underline{A B}}\right. \\
& \left.+\frac{1}{2}\left(H_{1}^{-}\right)_{i j}\left(H_{1}^{*}\right)^{i j}+\frac{\epsilon^{2} \lambda^{2}}{2}\left(4 m y^{2}-n y^{\prime 2}\right)\right], \\
\mathcal{L}_{\mathrm{WZ}}^{f i n}= & \int_{0}^{1} d t c^{2} \frac{2}{5 !} e_{0}^{\bar{A}_{1}} \cdots e_{0}^{\bar{A}_{5}} \mathrm{D}\left(t \bar{\theta}_{-}\right) \Gamma_{\bar{A}_{1} \cdots \bar{A}_{5}} \theta_{-}+C_{2}^{(6)} \\
= & d^{6} \xi \sqrt{s \operatorname{det} g_{0}}\left[-\bar{\theta}_{-} \gamma^{i} \mathrm{D}_{i} \theta_{-}\right]+C_{2}^{(6)} .
\end{aligned}
$$

Combining these results, we obtain the non-relativistic M5-brane action

$$
\begin{aligned}
S_{\mathrm{NR}}= & T_{\mathrm{NR}} \int d^{6} \xi \sqrt{s \operatorname{det} g_{0}}\left[\frac{1}{2} g_{0}^{i j} \partial_{i} y \underline{\underline{A}} \partial_{j} y^{\underline{B}} \eta_{\underline{A B}}+\frac{\epsilon^{2} \lambda^{2}}{2}\left(4 m y^{2}-n y^{\prime 2}\right)\right. \\
& \left.+\frac{1}{2}\left(H_{1}^{-}\right)_{i j}\left(H_{1}^{*}\right)^{i j}-2 \bar{\theta}_{-} \gamma^{i} \mathrm{D}_{i} \theta_{-}\right]+T_{\mathrm{NR}} \int_{\Sigma} C_{2}^{(6)} .
\end{aligned}
$$

In the flat limit $\lambda \rightarrow 0$, it is reduced to the linearized M5-brane action considered in [52].

\section{Summary and Discussions}

We have derived the NH superalgebra for AdS branes as IW contractions of the super$\mathrm{AdS} \times \mathrm{S}$ algebras in ten- and eleven-dimensions. Requiring that the isometry on the AdS brane worldvolume and the Lorentz symmetry in the transverse space extend to the superisometry, we classified possible branes. The NH superalgebra contains the super-isometry as a super-subalgebra: $\operatorname{su}(2 \mid 2) \times \operatorname{su}(2 \mid 2), \operatorname{osp}(4 \mid 4), \operatorname{osp}(6 \mid 2) \times \operatorname{psu}(2 \mid 1)$ and variants of them for non-relativistic AdS branes in $\operatorname{AdS}_{5} \times \mathrm{S}^{5}$, and $\operatorname{osp}(4 \mid 2) \times \operatorname{osp}(4 \mid 2), \operatorname{osp}(6 \mid 2) \times \operatorname{so}(2 \mid 2)$, $\operatorname{sp}(4 \mid 2) \times \operatorname{osp}(4 \mid 2), \operatorname{osp}(8 \mid 2) \times \operatorname{su}(2)$ and variants of them for non-relativistic AdS M-branes in $\mathrm{AdS}_{4 / 7} \times \mathrm{S}^{7 / 4}$. The possible branes are summarized in Table 1 and 4 . These contain $1 / 2$ BPS branes obtained by examining an open superstring in $\operatorname{AdS}_{5} \times \mathrm{S}^{5}$ and an open supermembrane in $\mathrm{AdS}_{4 / 7} \times \mathrm{S}^{7 / 4}$. We applied the similar analyses to branes in IIB ppwave and $\mathrm{M}$ pp-wave. The possible branes are summarized in Table 2, 3, 5 and 6 and we derived the NH superalgebras of these pp-wave branes.

The WZ terms of AdS branes in ten- and eleven-dimensions are examined by using the $\mathrm{CE}$ cohomology on the super-AdS $\times \mathrm{S}$ algebras. We find that WZ terms of the AdS branes in $\mathrm{AdS}_{5} \times \mathrm{S}^{5}$ and $\mathrm{AdS}_{4 / 7} \times \mathrm{S}^{7 / 4}$ are non-trivial elements of the CE cohomology except for those of strings in $\mathrm{AdS}_{5} \times \mathrm{S}^{5}$. 
By taking the non-relativistic limit of the relativistic brane actions obtained above, we derived non-relativistic $\mathrm{D} p$-brane actions in $\mathrm{AdS}_{5} \times \mathrm{S}^{5}$ and non-relativistic M-brane actions in $\mathrm{AdS}_{4 / 7} \times \mathrm{S}^{7 / 4}$. We have seen that there exists the consistent non-relativistic limit for $\mathrm{D} p$ (even,even) for $p=1 \bmod 4$ and $\mathrm{D} p$ (odd,odd) for $p=3 \bmod 4$ in $\mathrm{AdS}_{5} \times \mathrm{S}^{5}$, and M2(0,3), M2(2,1), M5(1,5) and M5(3,3) in $\mathrm{AdS}_{4} \times \mathrm{S}^{7}$ and $\mathrm{S}^{4} \times \mathrm{AdS}_{7}$. We derived the non-relativistic actions for these branes.

In the flat limit, the non-relativistic AdS D $p$ - and M2-brane actions are reduced to non-relativistic flat brane actions $[11,12]$. The non-relativistic AdS M5-brane action is reduced to the linearized M5-brane action [52]. It is interesting to examine these nonrelativistic AdS brane actions further, but is left for future investigations.

It is also interesting to examine the non-relativistic limit of branes in the pp-wave. It is known that the pp-wave superalgebra is an IW contraction of the AdS superalgebra. So, the brane actions in the pp-wave can be derived from those in the AdS background by expanding supercurrents with respect to the contraction parameter $\Lambda$ as was presented in Appendix C. Once having derived the brane action in the pp-wave one can easily extract the non-relativistic brane actions. These actions can be also derived from the non-relativistic actions derived in the present paper by expanding supercurrents with respect to the contraction parameter $\Omega$. We hope to report these points elsewhere in near future.

\section{Acknowledgments}

The authors thank Machiko Hatsuda for useful discussions and Kiyoshi Kamimura for useful comments. This work is supported in part by the Grant-in-Aid for Scientific Research (No. 17540262 and No. 17540091) from the Ministry of Education, Science and Culture, Japan. The work of K. Y. is supported in part by JSPS Research Fellowships for Young Scientists. 


\section{Appendix}

\section{A LI Cartan one forms}

\section{A.1 $\quad \mathbf{A d S}_{5} \times \mathbf{S}^{5}$}

Supervielbeins on the $\mathrm{AdS}_{5} \times S^{5}$ can be obtained via the coset construction with the coset supermanifold:

$$
\mathrm{AdS}_{5} \times S^{5} \sim \frac{P S U(2,2 \mid 4)}{S O(1,4) \times S O(4)} .
$$

We parametrize the group manifold as

$$
g=g_{x} g_{\theta}, \quad g_{\theta}=\mathrm{e}^{Q \theta}, \quad Q=\left(Q_{1}, Q_{2}\right), \quad \theta=\left(\begin{array}{c}
\theta_{1} \\
\theta_{2}
\end{array}\right) .
$$

where $g_{x}$ is concretely specified later. The supervielbeins $\mathbf{L}^{A}$ and $L^{\alpha}$, and super spin connection $\mathbf{L}^{A B}$ are the LI Cartan one forms defined by

$$
\begin{aligned}
g^{-1} d g & =\mathbf{L}^{A} P_{A}+\frac{1}{2} \mathbf{L}^{A B} J_{A B}+Q_{\alpha} L^{\alpha}, \\
g_{x}^{-1} d g_{x} & =e^{A} P_{A}+\frac{1}{2} \omega^{A B} J_{A B}
\end{aligned}
$$

where $e^{A}$ and $\omega^{A B}$ are the vielbein and the spin connection of the $\operatorname{AdS}_{5} \times \mathrm{S}^{5}$. After some algebra, we obtain 6

$$
\begin{aligned}
\mathbf{L}^{A} & =e^{A}+2 i \sum_{n=1}^{\infty} \bar{\theta} \Gamma^{A} \frac{\mathcal{M}^{2 n-2}}{(2 n) !} D \theta=e^{A}+2 i \bar{\theta} \Gamma^{A}\left(\frac{\cosh \mathcal{M}-1}{\mathcal{M}^{2}}\right) D \theta \\
L^{\alpha} & =\sum_{n=0}^{\infty} \frac{\mathcal{M}^{2 n}}{(2 n+1) !} D \theta=\frac{\sinh \mathcal{M}}{\mathcal{M}} D \theta \\
\mathbf{L}^{A B} & =\omega^{A B}-2 i \lambda \bar{\theta} \widehat{\Gamma}^{A B} i \sigma_{2} \sum_{n=1}^{\infty} \frac{\mathcal{M}^{2 n-2}}{(2 n) !} D \theta=\omega^{A B}-2 i \lambda \widehat{\theta} \widehat{\Gamma}^{A B} i \sigma_{2} \frac{\cosh \mathcal{M}-1}{\mathcal{M}^{2}} D \theta
\end{aligned}
$$

with

$$
\begin{aligned}
\mathcal{M}^{2} & =i \lambda\left(\widehat{\Gamma}_{A} i \sigma_{2} \theta \bar{\theta} \Gamma^{A}-\frac{1}{2} \Gamma_{A B} \theta \bar{\theta} \widehat{\Gamma}^{A B} i \sigma_{2}\right), \\
D \theta & =d \theta+\frac{\lambda}{2} e^{A} \widehat{\Gamma}_{A} i \sigma_{2} \theta+\frac{1}{4} \omega^{A B} \Gamma_{A B} \theta, \\
\widehat{\Gamma}_{A} & =\left(-\Gamma_{a} \mathcal{I}, \Gamma_{a^{\prime}} \mathcal{J}\right), \quad \widehat{\Gamma}_{A B}=\left(-\Gamma_{a b} \mathcal{I}, \Gamma_{a^{\prime} b^{\prime}} \mathcal{J}\right) .
\end{aligned}
$$

\footnotetext{
${ }^{6}$ The differential $d$ acts as $d(F \wedge G)=d F \wedge G+(-1)^{f} F \wedge d G$ (where $f$ is the degree of $F$ ), and commutes with $\theta$.
} 
The bosonic subalgebra is a direct product of $\mathrm{so}(2,4)$ and so(6), and so we may consider these parts separately. For an $(m, n)$-brane, it is convenient to parametrize the group manifold on the so $(2,4)$ algebra as

$$
g_{A d S}=g_{N} \mathrm{e}^{y^{\underline{\underline{a}}} P_{\underline{a}}}, \quad g_{N}=\mathrm{e}^{x^{\bar{a} m} P_{\bar{a}_{m}}} \ldots \mathrm{e}^{x^{\bar{a}_{1}} P_{\bar{a}_{1}}} .
$$

For this parametrization, we obtain

$$
\begin{aligned}
e^{\bar{a}_{\ell}} & =e_{N}^{\bar{a}_{\ell}} \cosh r_{y}, \quad \ell=1,2, \ldots, m, \\
e^{\underline{a}} & =\left(\frac{\sinh Y}{Y} d y\right)^{\underline{a}}, \\
\omega^{\bar{a}_{k} \bar{a}_{\ell}} & =\omega_{N}^{\bar{a}_{k} \bar{a}_{\ell}}, \\
\omega^{\underline{a} \bar{a}_{\ell}} & =-\lambda^{2} y^{\underline{a}} \frac{\sinh r_{y}}{r_{y}} e_{N}^{\bar{a}_{\ell}}, \\
\omega^{\underline{a b}} & =-2 \lambda^{2} y^{\underline{\underline{a}}}\left(\frac{\cosh Y-1}{Y^{2}} d y\right)^{\underline{b}]},
\end{aligned}
$$

where $r_{y}^{2}=\lambda^{2} y^{\underline{a}} y^{\underline{b}} \eta_{\underline{a} \underline{b}}=\lambda^{2} y^{2}$ and $\left(Y^{2}\right)^{\underline{a}} \underline{\underline{b}}=\lambda^{2}\left(y^{2} \delta_{\underline{b}}^{\underline{a}}-y^{\underline{a}} y_{\underline{b}}\right) . e_{N}$ and $\omega_{N}$ defined by

$$
g_{N}^{-1} d g_{N}=e_{N}^{\bar{a}} P_{\bar{a}}+\frac{1}{2} \omega_{N}^{\bar{a} \bar{b}} J_{\bar{a} \bar{b}}
$$

are obtained as

$$
\begin{aligned}
e_{N}^{\bar{a}_{\ell}} & =\cosh r_{1} \cdots \cosh r_{\ell-1} d x^{\bar{a}_{\ell}}, \\
\omega_{N}^{\bar{a}_{k} \bar{a}_{\ell}} & =-\lambda^{2} x^{\bar{a}_{k}} \frac{\sinh r_{k}}{r_{k}} \cosh r_{k+1} \cdots \cosh r_{\ell-1} d x^{\bar{a}_{\ell}}, \quad k<\ell
\end{aligned}
$$

where $r_{\ell}^{2}=\lambda^{2} x^{\bar{a}_{\ell}} x^{\bar{a}_{\ell}} \eta_{\bar{a}_{\ell} \bar{a}_{\ell}}$.

The vielbein $e^{a^{\prime}}$ and the spin connection $\omega^{a^{\prime} b^{\prime}}$ of $S^{5}$ are obtained as those of $\mathrm{AdS}_{5}$ with the replacement

$$
\lambda^{2} \rightarrow-\lambda^{2}, \quad \bar{a} \rightarrow \bar{a}^{\prime}, \quad \underline{a} \rightarrow \underline{a}^{\prime}, \quad m \rightarrow n .
$$

Under the scaling with $\Omega$ defined in (5.1), the above vielbeins and spin connections are expanded as

$$
\begin{aligned}
e^{\bar{a}_{\ell}} & =e_{0}^{\bar{a}_{\ell}}+\Omega^{2} e_{2}^{\bar{a}_{\ell}}+O\left(\Omega^{4}\right), \quad e_{0}^{\bar{a}_{\ell}}=e_{N}^{\bar{a}_{\ell}}, \quad e_{2}^{\bar{a}_{\ell}}=e_{N}^{\bar{a}_{\ell}} \frac{1}{2} r_{y}^{2}, \\
e^{\underline{a}} & =\Omega e_{1}^{\underline{a}}+O\left(\Omega^{3}\right), \quad e_{1}^{\underline{a}}=d y^{\underline{a}} \\
\omega^{\bar{a}_{k} \bar{a}_{\ell}} & =\omega_{0}^{\bar{a}_{k} \bar{a}_{\ell}}, \quad \omega_{0}^{\bar{a}_{k} \bar{a}_{\ell}}=\omega_{N}^{\bar{a}_{k} \bar{a}_{\ell}}, \\
\omega^{\underline{a} \bar{a}_{\ell}} & =-\Omega \lambda^{2} y^{\underline{\underline{a}}} e_{N}^{\bar{a}_{\ell}}+O\left(\Omega^{3}\right), \\
\omega^{\underline{a}} \underline{\underline{a}} & =-2 \Omega^{2} \lambda^{2} y^{\underline{\underline{a}}} d y^{\underline{b}]}+O\left(\Omega^{4}\right) .
\end{aligned}
$$




\section{A.2 $\quad \mathrm{AdS}_{4 / 7} \times \mathrm{S}^{7 / 4}$}

Supervielbeins on the $\mathrm{AdS}_{4 / 7} \times S^{7 / 4}$ can be obtained via the coset construction with the coset supermanifolds:

$$
\mathrm{AdS}_{4} \times S^{7} \sim \frac{O S p(8 \mid 4)}{S O(3,1) \times S O(7)}, \quad \mathrm{AdS}_{7} \times S^{4} \sim \frac{O S p\left(8^{*} \mid 4\right)}{S O(6,1) \times S O(4)} .
$$

Parametrizing the manifolds as $g(X, \theta)=g_{x} \mathrm{e}^{\theta Q}$, we obtain the expression of supervielbeins:

$$
\begin{aligned}
\mathbf{L}^{A} & =e^{A}-2 \bar{\theta} \Gamma^{A} \sum_{n=1} \frac{\mathcal{M}^{2 n-2}}{(2 n) !} D \theta, \\
\mathbf{L}^{A B} & =\omega^{A B}+2 \lambda \widehat{\Theta}^{A B} \sum_{n=1} \frac{\mathcal{M}^{2 n-2}}{(2 n) !} D \theta, \\
L & =\sum_{n=0} \frac{\mathcal{M}^{2 n}}{(2 n+1) !} D \theta,
\end{aligned}
$$

where we have introduced the following quantities:

$$
\begin{aligned}
(D \theta)^{\bar{\alpha}} & \equiv d \theta+-\frac{\lambda}{2} e^{A} \widehat{\Gamma}_{A} \theta+\frac{1}{4} \omega^{A B} \Gamma_{A B} \theta \\
\mathcal{M}^{2} & =\lambda\left(\widehat{\Gamma}^{A} \theta \bar{\theta} \Gamma_{A}+\frac{1}{2} \Gamma^{A B} \theta \widehat{\theta} \widehat{\Gamma}_{A B}\right) \\
\widehat{\Gamma}_{A} & =\left(2 \mathcal{I} \Gamma_{a}, \mathcal{I} \Gamma_{a^{\prime}}\right), \quad \widehat{\Gamma}_{A B}=\left(2 \mathcal{I} \Gamma_{a b}, \mathcal{I} \Gamma_{a^{\prime} b^{\prime}}\right) .
\end{aligned}
$$

Here $e_{M}^{A}$ and $\omega_{M}^{A B}$ are the vielbein and the spin connection, respectively.

Since the bosonic subalgebra is the direct product of $\mathrm{so}(3,2)(\mathrm{so}(5))$ and $\mathrm{so}(8)(\mathrm{so}(6,2))$, we may consider these parts separately as in the case of $\operatorname{AdS}_{5} \times \mathrm{S}^{5}$. For the former group manifold, a group element is represented by

$$
g_{4}=g_{N} e^{y^{\underline{\underline{a}}} P_{\underline{a}}}, \quad g_{N}=\mathrm{e}^{x^{\bar{a}} m P_{\bar{a}_{m}}} \cdots \mathrm{e}^{x^{\bar{a}_{1}} P_{\bar{a}_{1}}} .
$$

It is straightforward to derive

$$
\begin{aligned}
e^{\bar{a}_{\ell}} & =e_{N}^{\bar{a}_{\ell}} \cosh r_{y}, \quad \ell=1, \cdots, m \\
e^{\underline{a}} & =\left(\frac{\sinh Y}{Y} d y\right)^{\underline{a}}, \\
\omega^{\bar{a}_{k} \bar{a}_{\ell}} & =\omega_{N}^{\bar{a}_{k} \bar{a}_{\ell}}, \\
\omega^{\underline{a b}} & =-8 \epsilon^{2} \lambda^{2} y^{\underline{\underline{a}}}\left(\frac{\cosh Y-1}{Y} d y\right)^{\underline{b}}, \\
\omega^{\bar{a}_{\ell} \underline{b}} & =4 \epsilon^{2} \lambda^{2} e_{N}^{\bar{a}_{\ell}} y^{\underline{\underline{b}}} \frac{\sinh r_{y}}{r_{y}}
\end{aligned}
$$


with

$$
\begin{aligned}
e_{N}^{\bar{a}_{\ell}} & =d x^{\bar{a}_{\ell}} \cosh r_{1} \cdots \cosh r_{\ell-1}, \\
\omega_{N}^{\bar{a}_{k} \bar{a}_{\ell}} & =-4 \epsilon^{2} \lambda^{2} x^{\bar{a}_{k}} d x^{\bar{a}_{\ell}} \frac{\sinh r_{k}}{r_{k}} \cosh r_{k+1} \cdots \cosh r_{\ell-1}, \quad k<\ell,
\end{aligned}
$$

where $r_{k}^{2}=4 \epsilon^{2} \lambda^{2} x^{\bar{a}_{k}} x^{\bar{a}_{k}} \eta_{\bar{a}_{k} \bar{a}_{k}}, r_{y}^{2}=4 \epsilon^{2} \lambda^{2} y^{\underline{a}} y^{\underline{b}} \eta_{\underline{a b}}=4 \epsilon^{2} \lambda^{2} y^{2}$ and $Y^{2}=4 \epsilon^{2} \lambda^{2}\left(y^{2} \delta_{\underline{b}}^{\underline{a}}-y^{\underline{a}} y_{\underline{b}}\right)$. For the latter group manifold, the vielbein and the spin connection can be obtained from those for the former case with the replacement

$$
\epsilon^{2} \rightarrow-\frac{1}{4} \epsilon^{2}, \quad \bar{a} \rightarrow \bar{a}^{\prime}, \quad \underline{a} \rightarrow \underline{a}^{\prime}, \quad m \rightarrow n .
$$

Under the $\Omega$-scaling (9.1), these scale as

$$
\begin{aligned}
e^{\bar{a}} & =e_{0}^{\bar{a}}+\Omega^{2} e_{2}^{\bar{a}}+O\left(\Omega^{4}\right), \quad e_{0}^{\bar{a}}=e_{N}^{\bar{a}}, \quad e_{2}^{\bar{a}}=e_{N}^{\bar{a}} \frac{r_{y}^{2}}{2} \\
e^{\underline{a}} & =\Omega e_{1}^{\underline{a}}+O\left(\Omega^{3}\right), \quad e_{1}^{\underline{a}}=d y^{\underline{a}}, \\
\omega^{\bar{a} \bar{b}} & =\omega_{0}^{\bar{a} \bar{b}}, \quad \omega_{0}^{\bar{a} \bar{b}}=\omega_{N}^{\bar{a} \bar{b}}, \\
\omega^{\underline{a} b} & =O\left(\Omega^{2}\right), \\
\omega^{\bar{a} \underline{b}} & =\Omega 4 \epsilon^{2} \lambda^{2} e_{N}^{\bar{a}} y^{\underline{b}}+O\left(\Omega^{3}\right) .
\end{aligned}
$$

\section{B $\kappa$-symmetry}

\section{B.1 D-branes in $\mathrm{AdS}_{5} \times \mathrm{S}^{5}$}

Here we recall the $\kappa$-variation of the action (4.1) by following $[36,40,41]$. Here we consider both Lorentzian branes and Euclidean branes.

Following (2.6), one can derive a variation of the supercurrents by using the homotopy formula as follows:

$$
\begin{aligned}
\delta \mathbf{L}^{A} & =d \delta x^{A}+\eta_{B C} \mathbf{L}^{B} \delta x^{C A}+\eta_{B C} \mathbf{L}^{A B} \delta x^{C}-2 i \bar{L} \Gamma^{A} \delta \theta, \\
\delta L & =d \delta \theta-\frac{\lambda}{2} \delta x^{A} \widehat{\Gamma}_{A} i \sigma_{2} L+\frac{\lambda}{2} \mathbf{L}^{A} \widehat{\Gamma}_{A} i \sigma \delta \theta, \\
\delta \mathbf{L}^{a b} & =d \delta x^{a b}-2 \lambda^{2} \mathbf{L}^{a} \delta x^{b}+2 \eta_{c d} \mathbf{L}^{a c} \delta x^{d b}+2 i \lambda \bar{L} \widehat{\Gamma}^{a b} i \sigma_{2} \delta \theta, \\
\delta \mathbf{L}^{a^{\prime} b^{\prime}} & =d \delta x^{a^{\prime} b^{\prime}}+2 \lambda^{2} \mathbf{L}^{a^{\prime}} \delta x^{b^{\prime}}+2 \eta_{c^{\prime} d^{\prime}} \mathbf{L}^{a^{\prime} c^{\prime}} \delta x^{d^{\prime} b^{\prime}}+2 i \lambda \bar{L} \widehat{\Gamma}^{a^{\prime} b^{\prime}} i \sigma_{2} \delta \theta,
\end{aligned}
$$

where

$$
\delta x^{A}=\delta Z^{\hat{M}} \mathbf{L}_{\hat{M}}^{A}, \quad \delta x^{A B}=\delta Z^{\hat{M}} \mathbf{L}_{\hat{M}}^{A B}, \quad \delta \theta^{\alpha}=\delta Z^{\hat{M}} L_{\hat{M}}^{\alpha} .
$$


A universal feature of the $\kappa$-variation is

$$
\delta_{\kappa} x^{A}=0
$$

By using (B.1), one can find that

$$
\delta_{\kappa} g_{i j}=-4 i \mathbf{L}_{(i}^{A} \bar{L}_{j)} \Gamma_{A} \delta \theta
$$

and

$$
\delta_{\kappa} d \mathcal{F}=-2 i d\left(\mathbf{L}^{A} \bar{L} \Gamma_{A} \sigma \delta \theta\right) \quad \rightarrow \quad \delta_{\kappa} \mathcal{F}=-2 i \mathbf{L}^{A} \bar{L} \Gamma_{A} \sigma \delta \theta
$$

where the exact term is deleted by $\delta_{\kappa} A$. By using (B.1) and (B.5), we find that

$$
\begin{aligned}
\delta_{\kappa} h_{p+2} & =d\left[\mathcal{C}_{\kappa} \wedge e^{\mathcal{F}}\right]_{p+1}, \quad \mathcal{C}_{\kappa}=\bigoplus_{\ell=\text { even }} \mathcal{C}_{\kappa}^{(\ell)} \\
\mathcal{C}_{\kappa}^{(2 n)} & =\frac{2 \sqrt{s}}{(2 n-1) !} \mathbf{L}^{A_{1}} \cdots \mathbf{L}^{A_{2 n-1}} \bar{L} \Gamma_{A_{1} \cdots A_{2 n-1}}(\sigma)^{n} i \sigma_{2} \delta_{\kappa} \theta
\end{aligned}
$$

so that

$$
\delta_{\kappa} \mathcal{L}_{W Z}=\left[\mathcal{C}_{\kappa} \wedge e^{\mathcal{F}}\right]_{p+1}
$$

where $[\bullet]_{p+1}$ represents the $(p+1)$-form part of $\bullet$.

By using these expressions, one finds that the action (4.1) is invariant under

$$
\begin{aligned}
\delta_{\kappa} \theta= & (1+\Gamma) \kappa, \\
\Gamma= & \frac{s \sqrt{-s}}{\sqrt{s \operatorname{det}(g+\mathcal{F})}} \sum_{n=0} \frac{1}{2^{n} n !} \gamma^{j_{1} k_{1} \cdots j_{n} k_{n}} \mathcal{F}_{j_{1} k_{1}} \cdots \mathcal{F}_{j_{n} k_{n}} \\
& \times(-1)^{n}(\sigma)^{n-\frac{p-3}{2}} i \sigma_{2} \frac{1}{(p+1) !} \epsilon^{i_{1} \cdots i_{p+1}} \gamma_{i_{1} \cdots i_{p+1}},
\end{aligned}
$$

where $\gamma_{i}=\mathbf{L}_{i}^{A} \Gamma_{A}$.

Under the $\Omega$-scaling, $\Gamma$ is expanded as

$$
\begin{aligned}
\Gamma & =\Gamma_{0}+O(\Omega) \\
\Gamma_{0} & =\frac{s \sqrt{-s}}{\sqrt{s \operatorname{det} g_{0}}} \frac{1}{(p+1) !} \epsilon^{i_{1} \cdots i_{p+1}}\left(\mathbf{L}_{0}^{\bar{A}_{0}}\right)_{i_{1}} \cdots\left(\mathbf{L}_{0}^{\bar{A}_{p}}\right)_{i_{p+1}} \Gamma_{\bar{A}_{0} \cdots \bar{A}_{p}}(\sigma)^{-\frac{p-3}{2}} i \sigma_{2} \\
& =M .
\end{aligned}
$$

Expanding $\kappa$ as

$$
\kappa=\kappa_{+}+\Omega \kappa_{-}, \quad \kappa_{ \pm}=\mathcal{P}_{ \pm} \kappa_{ \pm}
$$


leads to

$$
\delta_{\kappa} \theta_{+}=\left(1+\Gamma_{0}\right) \kappa_{+}=2 \kappa_{+} .
$$

This implies that the $\kappa$-symmetry can be gauge fixed by choosing $\theta_{+}=0$ since $\left.\delta_{\kappa} \theta_{+}\right|_{\theta_{+}=0}=$ $2 \kappa_{+}$.

For an F-string, we obtain the similar expression with $\sigma=-\sigma_{1}$ and $\mathcal{F}=0$. Hence the action is $\kappa$-invariant, and the $\kappa$-gauge symmetry is fixed by $\theta_{+}=0$.

\section{B.2 M-brane in $\mathrm{AdS}_{4 / 7} \times \mathbf{S}^{7 / 4}$}

Following $[48,49,51]$, we recall the $\kappa$-symmetry of the M-brane actions. Here we shall consider Euclidean branes as well as Lorentzian branes.

A variation of the supercurrents is derived from (6.5) as

$$
\begin{aligned}
\delta \mathbf{L}^{A} & =d \delta x^{A}-\eta_{B C} \delta x^{A B} \mathbf{L}^{C}+\eta_{B C} \mathbf{L}^{A B} \delta x^{C}+2 \bar{L} \Gamma^{A} \delta \theta \\
\delta \mathbf{L}^{a b} & =d \delta x^{a b}+8 \epsilon^{2} \lambda^{2} \mathbf{L}^{a} \delta x^{b}+2 \eta_{c d} \mathbf{L}^{c a} \delta x^{b d}-2 \lambda \bar{L} \widehat{\Gamma}^{a b} \delta \theta \\
\delta \mathbf{L}^{a^{\prime} b^{\prime}} & =d \delta x^{a^{\prime} b^{\prime}}-2 \epsilon^{2} \lambda^{2} \mathbf{L}^{a^{\prime}} \delta x^{b^{\prime}}+2 \eta_{c^{\prime} d^{\prime}} \mathbf{L}^{c^{\prime} a^{\prime}} \delta x^{b^{\prime} d^{\prime}}-2 \lambda \bar{L} \widehat{\Gamma}^{a^{\prime} b^{\prime}} \delta \theta, \\
\delta L & =d \delta \theta+\frac{1}{2} \delta x^{A} \widehat{\Gamma}_{A} L-\frac{\lambda}{2} \mathbf{L}^{A} \widehat{\Gamma}_{A} \delta \theta-\frac{1}{4} \delta x^{A B} \Gamma_{A B} L+\frac{1}{4} \mathbf{L}^{A B} \Gamma_{A B} \delta \theta,
\end{aligned}
$$

where $\delta x^{A}, \delta x^{A B}$ and $\delta \theta$ have been defined in (B.2). For the $\kappa$-variation, we require that $\delta_{\kappa} x^{A}=0$.

\section{B.2.1 M2-brane}

Let us first consider the case of an M2-brane. From (B.14), one can obtain

$$
\delta_{\kappa} g_{i j}=4 \mathbf{L}_{(i}^{A} \bar{L}_{j)} \Gamma_{A} \delta_{\kappa} \theta
$$

and

$$
\delta_{\kappa} h_{4}=d\left(-c \mathcal{L}^{A} \mathbf{L}^{B} \bar{L} \Gamma_{A B} \delta_{\kappa} \theta\right) \quad \rightarrow \quad \delta_{\kappa} \mathcal{L}_{\mathrm{WZ}}^{M 2}=-c \mathcal{L}^{A} \mathbf{L}^{B} \bar{L} \Gamma_{A B} \delta_{\kappa} \theta
$$

By using them one can see that the action (8.1) is invariant under

$$
\delta_{\kappa} \theta=(1+\Gamma) \kappa, \quad \Gamma=\frac{i \sqrt{s}}{\sqrt{s \operatorname{det} g}} \frac{1}{3 !} \epsilon^{i j k} \gamma_{i j k} .
$$

Under the $\Omega$-scaling, $\Gamma$ is expanded as

$$
\Gamma=\Gamma_{0}+O\left(\Omega^{2}\right), \quad \Gamma_{0}=i \sqrt{s} \Gamma_{\bar{A}_{0} \bar{A}_{1} \bar{A}_{2}}=M
$$


By expanding $\kappa$ as (B.12), we derive

$$
\delta_{\kappa} \theta_{+}=\left(1+\Gamma_{0}\right) \kappa_{+}=2 \kappa_{+}
$$

which implies that the $\kappa$-gauge symmetry is fixed by $\theta_{+}=0$.

\section{B.2.2 M5-brane}

Next, we consider the case of an M5-brane. A variation of $\mathcal{H}$ is taken with (B.14 $)$ as follows:

$$
-c \delta_{\kappa} d \mathcal{H}=\delta_{\kappa} h_{4}=d\left(-c \mathbf{L}^{A} \mathbf{L}^{B} \bar{L} \Gamma_{A B} \delta_{\kappa} \theta\right) \quad \rightarrow \quad \delta_{\kappa} \mathcal{H}=\mathbf{L}^{A} \mathbf{L}^{B} \bar{L} \Gamma_{A B} \delta_{\kappa} \theta
$$

where the exact term is deleted by $\delta_{\kappa} B$. Noting that

$$
\delta_{\kappa} h_{7}=c^{2} d\left[\frac{2}{5 !} \mathbf{L}^{A_{1}} \cdots \mathbf{L}^{A_{5}} \bar{L} \Gamma_{A_{1} \cdots A_{5}} \delta_{\kappa} \theta+\frac{1}{2} \mathbf{L}^{A} \mathbf{L}^{B} \bar{L} \Gamma_{A B} \delta_{\kappa} \theta \mathcal{H}\right]
$$

we see that

$$
\delta_{\kappa} \mathcal{L}_{\mathrm{WZ}}=c^{2}\left[\frac{2}{5 !} \mathbf{L}^{A_{1}} \cdots \mathbf{L}^{A_{5}} \bar{L} \Gamma_{A_{1} \cdots A_{5}} \delta_{\kappa} \theta+\frac{1}{2} \mathbf{L}^{A} \mathbf{L}^{B} \bar{L} \Gamma_{A B} \delta_{\kappa} \theta \mathcal{H}\right],
$$

where $c^{2}=i \sqrt{s}$. The $\kappa$-invariance of the action is shown by following [51] (see also [52]). By using the expressions derived above and the following useful relations

$$
\begin{aligned}
& \bar{\gamma}=\frac{s \sqrt{-s}}{6 ! \sqrt{s \operatorname{det} g}} \epsilon^{i_{1} \cdots i_{6}} \gamma_{i_{1} \cdots i_{6}}, \quad \bar{\gamma}^{2}=1, \\
& \epsilon^{i_{1} \cdots i_{6-n} j_{1} \cdots j_{n}} \gamma_{j_{1} \cdots j_{n}}=(-1)^{\left[\frac{6-n}{2}\right]} n ! \frac{s \sqrt{-s}}{\sqrt{s \operatorname{det} g}} \gamma^{i_{1} \cdots i_{6-n}} \bar{\gamma}, \\
& \epsilon^{i_{1} \cdots i_{6-n} k_{1} \cdots k_{n}} \epsilon_{j_{1} \cdots j_{6-n} k_{1} \cdots k_{n}}=s n !(6-n) ! \delta_{\left[j_{1}\right.}^{i_{1}} \cdots \delta_{\left.j_{6-n}\right]}^{i_{6-n}}, \\
& \mathcal{H}_{i j k}=3 \mathcal{H}_{[i j} v_{k]}-\frac{s}{2} \sqrt{s \operatorname{det} g} \epsilon_{i j k l m n} \mathcal{H}^{* l m} v^{n},
\end{aligned}
$$

it is shown that the M5-brane action is invariant under the $\kappa$-variation

$$
\begin{aligned}
\delta_{\kappa} \theta & =(1+\Gamma) \kappa, \quad \delta_{\kappa} a=0, \\
\Gamma & =\frac{\sqrt{s \operatorname{det} g}}{\sqrt{s \operatorname{det}\left(g-i c^{2} \mathcal{H}^{*}\right)}}\left(\bar{\gamma}-\frac{c^{2}}{2} \mathcal{H}_{i j}^{*} v_{k} \gamma^{i j k}-\frac{s c^{2}}{16 \sqrt{s \operatorname{det} g}} \epsilon^{i_{1} \cdots i_{6}} \mathcal{H}_{i_{1} i_{2}}^{*} \mathcal{H}_{i_{3} i_{4}}^{*} \gamma_{i_{5} i_{6}}\right) .
\end{aligned}
$$

Under the $\Omega$-scaling, $\Gamma$ is expanded as

$$
\begin{aligned}
\Gamma & =\Gamma_{0}+O(\Omega), \\
\Gamma_{0} & =\frac{s \sqrt{-s}}{\sqrt{s \operatorname{det} g_{0}}} \frac{1}{6 !} \epsilon^{i_{1} \cdots i_{6}}\left(\mathbf{L}_{0}^{\bar{A}_{1}}\right)_{i_{1}} \cdots\left(\mathbf{L}_{0}^{\bar{A}_{6}}\right)_{i_{6}} \Gamma_{\bar{A}_{1} \cdots \bar{A}_{6}}=s \sqrt{-s} \Gamma_{\bar{A}_{0} \cdots \bar{A}_{5}}=M,
\end{aligned}
$$

which implies that

$$
\delta_{\kappa} \theta_{+}=\left(1+\Gamma_{0}\right) \kappa_{+}=2 \kappa_{+} .
$$

Thus the $\kappa$-symmetry is gauge fixed by $\theta_{+}=0$. 


\section{Penrose limit of Brane Actions}

Here we will construct the action of D-branes and M-branes on pp-wave backgrounds via the Penrose limit, instead of non-relativistic limit. This is a natural application of our procedure. The Penrose limit of an alternative action of an AdS superstring has been discussed in [25]. The result includes Metsaev's results for F-string [53] and D3-brane [54] on the maximally supersymmetric pp-wave.

\section{C.1 Branes in IIB pp-wave}

We derive the $\mathrm{D} p$-brane action in the IIB pp-wave from the $\mathrm{D} p$-brane action in $\mathrm{AdS}_{5} \times \mathrm{S}^{5}$

$$
\begin{aligned}
S & =T \int \mathcal{L}_{D B I}+\mathcal{L}_{W Z}, \\
\mathcal{L}_{\mathrm{DBI}}= & \sqrt{s \operatorname{det}(g+\mathcal{F})} d^{p+1} \xi, \quad d \mathcal{L}_{\mathrm{WZ}}=h_{p+2}=\sum_{n=0} h^{(p+2-2 n)} \mathcal{F}^{n}, \\
h^{(2 n+1)}= & \frac{c}{(2 n-1) !}\left[\mathbf{L}^{A_{1}} \cdots \mathbf{L}^{A_{2 n-1}} \bar{L} \Gamma_{A_{1} \cdots A_{2 n-1}} \sigma^{n+1} \varrho L\right. \\
& \left.\quad+\delta_{n, 2} \frac{i}{5} \lambda\left(\epsilon_{a_{1} \cdots a_{5}} \mathbf{L}^{a_{1}} \cdots \mathbf{L}^{a_{5}}-\epsilon_{a_{1}^{\prime} \cdots a_{5}^{\prime}} \mathbf{L}^{a_{1}^{\prime}} \cdots \mathbf{L}^{a_{5}^{\prime}}\right)\right]
\end{aligned}
$$

where $\sigma=\sigma_{3}$ and $\varrho=\sigma_{1} . c=\sqrt{s}$ is required by the $\kappa$-invariance of the action.

The Penrose limit considered in section 3 is equivalent to scaling the coordinates as

$$
X^{+} \rightarrow \Lambda^{2} X^{+}, \quad X^{\hat{i}} \rightarrow \Lambda X^{\hat{i}}, \quad \theta_{+} \rightarrow \Lambda \theta_{+}
$$

and taking the limit $\Lambda \rightarrow 0$. Under the scaling, LI Cartan one-forms are expanded as

$$
\begin{aligned}
\mathbf{L}^{+} & =\sum_{n=0} \Lambda^{2 n+2} \mathbf{L}_{2 n+2}^{+}, \quad \mathbf{L}^{\hat{i}}=\sum_{n=0} \Lambda^{2 n+1} \mathbf{L}_{2 n+1}^{\hat{i}}, \quad \mathbf{L}_{*}^{\hat{i}}=\sum_{n=0} \Lambda^{2 n+1} \mathbf{L}_{* 2 n+1}^{\hat{i}}, \\
L_{+} & =\sum_{n=0} \Lambda^{2 n+1} L_{+2 n+1}
\end{aligned}
$$

where

$$
\mathbf{L}^{ \pm}=\frac{1}{\sqrt{2}}\left(\mathbf{L}^{9} \pm \mathbf{L}^{0}\right), \quad L_{ \pm}=\ell_{ \pm} L_{ \pm}, \quad \mathbf{L}_{*}^{\hat{i}}=\left(\mathbf{L}^{0 i}, \mathbf{L}^{9 i^{\prime}}\right)
$$

Under the expansion (C.3), we derive

$$
g_{i j}=\Lambda^{2} g_{i j}^{(p p)}+O\left(\Lambda^{4}\right), \quad g_{i j}^{(p p)}=2\left(\mathbf{L}_{2}^{+}\right)_{i}\left(\mathbf{L}_{0}^{-}\right)_{j}+\left(\mathbf{L}_{1}^{\hat{i}}\right)_{i}\left(\mathbf{L}_{1}^{\hat{j}}\right)_{j} \eta_{\hat{i} \hat{j}}
$$


It follows from ${ }^{7}$

$$
d \mathcal{F}=\Lambda^{2}\left[-i \mathbf{L}^{+} \bar{L}_{-} \Gamma_{+} \sigma L_{-}-i \mathbf{L}^{-} \bar{L}_{+} \Gamma_{-} \sigma L_{+}-2 i \mathbf{L}^{\hat{i}} \bar{L}_{+} \Gamma_{\hat{i}} \sigma L_{-}\right]
$$

that

$$
\mathcal{F}=\Lambda^{2} \mathcal{F}_{p p}+O\left(\Lambda^{4}\right)
$$

where we assume that $F=\Lambda^{2} F_{p p}$. These imply that

$$
\mathcal{L}_{\mathrm{DBI}}=\Lambda^{p+1} \mathcal{L}_{\mathrm{DBI}}^{p p}+O\left(\Lambda^{p+3}\right), \quad \mathcal{L}_{\mathrm{DBI}}^{p p}=\sqrt{s \operatorname{det}\left(g_{p p}+\mathcal{F}_{p p}\right)} d^{p+1} \xi
$$

The factor $\Lambda^{p+1}$ is absorbed into the definition of the tension as

$$
T=\Lambda^{-(p+1)} T_{p p}
$$

One finds that the fermionic part of $h^{(2 n+1)}$ is scaled as

$$
\begin{aligned}
\left.h^{(2 n+1)}\right|_{\text {fermionic }}= & \left.\Lambda^{2 n} h_{p p}^{(2 n+1)}\right|_{\text {fermionic }}+O\left(\Lambda^{2 n+2}\right) \\
\left.h_{p p}^{(2 n+1)}\right|_{\text {fermionic }}= & \frac{c}{(2 n-1) !} \mathbf{L}^{A_{1}} \cdots \mathbf{L}^{A_{2 n-1}} \bar{L} \Gamma_{A_{1} \cdots A_{2 n-1}} \sigma_{3}^{n+1} \sigma_{1} L \\
= & \frac{c}{(2 n-1) !} \mathbf{L}^{\hat{i}_{1}} \cdots \mathbf{L}^{\hat{i}_{2 n-1}}\left(\bar{L}_{+} \Gamma_{\hat{i}_{1} \cdots \hat{i}_{2 n-1}} \sigma_{3}^{n+1} \sigma_{1} L_{-}\right. \\
& \left.\quad+\bar{L}_{-} \Gamma_{\hat{i}_{1} \cdots \hat{i}_{2 n-1}} \sigma_{3}^{n+1} \sigma_{1} L_{+}\right) \\
& +\frac{c}{(2 n-2) !} \mathbf{L}^{+} \mathbf{L}^{\hat{i}_{1}} \cdots \mathbf{L}^{\hat{i}_{2 n-2}} \bar{L}_{-} \Gamma_{+\hat{i}_{1} \cdots \hat{i}_{2 n-2}} \sigma_{3}^{n+1} \sigma_{1} L_{-} \\
& +\frac{c}{(2 n-2) !} \mathbf{L}^{-} \mathbf{L}^{\hat{i}_{1}} \cdots \mathbf{L}^{\hat{i}_{2 n-2}} \bar{L}_{+} \Gamma_{-\hat{i}_{1} \cdots \hat{i}_{2 n-2}} \sigma_{3}^{n+1} \sigma_{1} L_{+} \\
& +\frac{c}{(2 n-3) !} \mathbf{L}^{+} \mathbf{L}^{-} \mathbf{L}^{\hat{i}_{1}} \cdots \mathbf{L}^{\hat{i}_{2 n-3}}\left(\bar{L}_{-} \Gamma_{\hat{i}_{1} \cdots \hat{i}_{2 n-3}} \sigma_{3}^{n+1} \sigma_{1} L_{+}\right. \\
& \left.\quad-\bar{L}_{+} \Gamma_{\hat{i}_{1} \cdots \hat{i}_{2 n-3}} \sigma_{3}^{n+1} \sigma_{1} L_{-}\right) .
\end{aligned}
$$

For the bosonic part, we derive

$$
\begin{aligned}
& \left.h^{(5)}\right|_{\text {bosonic }}=\left.\Lambda^{4} h_{p p}^{(5)}\right|_{\text {bosonic }}+O\left(\Lambda^{6}\right), \\
& \left.h_{p p}^{(5)}\right|_{\text {bosonic }}=-i c \frac{4 \lambda}{\sqrt{2}} \mathbf{L}^{-}\left(\mathbf{L}^{1} \mathbf{L}^{2} \mathbf{L}^{3} \mathbf{L}^{4}+\mathbf{L}^{5} \mathbf{L}^{6} \mathbf{L}^{7} \mathbf{L}^{8}\right) .
\end{aligned}
$$

The $O\left(\Lambda^{6}\right)$-term which contains $\mathbf{L}^{+}$disappears in the limit.

To summarize the pp-wave $\mathrm{D} p$-brane action is given as

$$
S_{p p}=T_{p p} \int \mathcal{L}_{\mathrm{DBI}}^{p p}+\mathcal{L}_{\mathrm{WZ}}^{p p}
$$

\footnotetext{
${ }^{7} \mathbf{L}^{+}, \mathbf{L}^{-}, \mathbf{L}^{\hat{i}}, L_{+}$and $L_{-}$are understood as $\mathbf{L}_{2}^{+}, \mathbf{L}_{0}^{-}, \mathbf{L}_{1}^{\hat{i}}, L_{+1}$ and $L_{-0}$ respectively below.
} 
with (C.8) and

$$
\begin{aligned}
h_{p+2}^{p p}= & d \mathcal{L}_{W Z}^{p p}=\sum_{n=0} h_{p p}^{(p+2-2 n)} \mathcal{F}_{p p}^{n}, \\
h_{p p}^{(2 n+1)}= & \frac{c}{(2 n-1) !} \mathbf{L}^{A_{1}} \cdots \mathbf{L}^{A_{2 n-1}} \bar{L} \Gamma_{A_{1} \cdots A_{2 n-1}} \sigma^{n+1} \varrho L \\
& -i c \delta_{n, 2} \frac{4 \lambda}{\sqrt{2}} \mathbf{L}^{-}\left(\mathbf{L}^{1} \mathbf{L}^{2} \mathbf{L}^{3} \mathbf{L}^{4}+\mathbf{L}^{5} \mathbf{L}^{6} \mathbf{L}^{7} \mathbf{L}^{8}\right) .
\end{aligned}
$$

This reproduces the pp-wave D3-brane action given in [54] as the $p=3$ case. Let $\varrho=\sigma_{3}$ and $\sigma=-\sigma_{1}$ and replace $\mathcal{L}_{\mathrm{DBI}}$ with $\mathcal{L}_{\mathrm{NG}}$ or with the Polyakov action, then it is reduced to the pp-wave F-string action constructed in [53].

The $(p+2)$-form $h_{p+2}^{p p}$ can be shown to be a non-trivial element of the CE cohomology except for $h_{3}$ by following the procedure explained in section 4.1. It is easy to obtain the $(p+1)$-dimensional form of the $\mathrm{WZ}$ term as was done in section 4.2.

\section{C.2 Branes in M pp-wave}

The Penrose limit considered in section 7 is equivalent to scaling the coordinates as (C.2) and taking the limit $\Lambda \rightarrow 0$. Under the scaling, LI Cartan one-forms are expanded as (C.3) where we define Cartan one-forms as

$$
\mathbf{L}^{ \pm}=\frac{1}{\sqrt{2}}\left(\mathbf{L}^{\natural} \pm \mathbf{L}^{0}\right), \quad \mathbf{L}_{*}^{\hat{i}}=\left\{\begin{array}{l}
\left(\mathbf{L}^{i 0}, \mathbf{L}^{i^{\prime} \natural}\right) \text { for } \mathrm{AdS}_{4} \times \mathrm{S}^{7} \\
\left(\mathbf{L}^{i \natural}, \mathbf{L}^{i^{\prime} 0}\right) \text { for } \mathrm{AdS}_{7} \times \mathrm{S}^{4}
\end{array} \quad L_{ \pm}=\ell_{ \pm} L\right.
$$

\section{C.2.1 Penrose limit of M2 brane action}

We consider the Penrose limit of the M2-brane action

$$
\begin{aligned}
S & =T \int \mathcal{L}_{\mathrm{NG}}+\mathcal{L}_{\mathrm{WZ}} \\
\mathcal{L}_{\mathrm{NG}} & =\sqrt{s \operatorname{det} g} \\
d \mathcal{L}_{\mathrm{WZ}} & =h_{4}=c\left[\frac{1}{2} \mathbf{L}^{A} \mathbf{L}^{B} \bar{L} \Gamma_{A B} L-\frac{6 \lambda}{4 !} \epsilon_{a_{1} \cdots a_{4}} \mathbf{L}^{a_{1}} \cdots \mathbf{L}^{a_{4}}\right]
\end{aligned}
$$

where $c=i \sqrt{s}$ is required by the $\kappa$-invariance of the action. Under the expansion (C.3), $\mathcal{L}_{\mathrm{NG}}$ is expanded as

$$
\mathcal{L}_{\mathrm{NG}}=\Lambda^{3} \mathcal{L}_{\mathrm{NG}}^{p p}+O\left(\Lambda^{5}\right), \quad \mathcal{L}_{\mathrm{NG}}^{p p}=\sqrt{s \operatorname{det} g_{p p}}
$$


while $d \mathcal{L}_{\mathrm{WZ}}=h_{4}$ is expanded as

$$
\begin{aligned}
h_{4} & =\Lambda^{3} h_{4}^{p p}+O\left(\Lambda^{5}\right), \\
h_{4}^{p p} & =c\left[\frac{1}{2} \mathbf{L}^{A} \mathbf{L}^{B} \bar{L} \Gamma_{A B} L+\frac{6 \lambda}{\sqrt{2}} \mathbf{L}^{-} \mathbf{L}^{1} \mathbf{L}^{2} \mathbf{L}^{3} \mathbf{L}^{4}\right] .
\end{aligned}
$$

The $\Lambda^{3}$ factor is absorbed into the definition of the tension as $T=\Lambda^{-3} T_{p p}$. The pp-wave M2-brane action is given as

$$
S=T_{p p} \int \mathcal{L}_{\mathrm{NG}}^{p p}+\mathcal{L}_{W Z}^{p p}
$$

with (C.21) and $h_{4}^{p p}=d \mathcal{L}_{W Z}^{p p}$ with (C.23).

\section{C.2.2 Penrose limit of M5-brane action}

Next we will consider the M5-brane action

$$
S=T \int \mathcal{L}_{\mathrm{PST}}+\mathcal{L}_{\mathrm{WZ}},
$$

with

$$
\begin{aligned}
\mathcal{L}_{\mathrm{PST}} & =\sqrt{s \operatorname{det}\left(g_{i j}-i c^{2} \mathcal{H}_{i j}^{*}\right)}+c^{2} \frac{\sqrt{s \operatorname{det} g}}{4} \mathcal{H}^{* i j} \mathcal{H}_{i j}, \\
\mathcal{H}_{i j} & =\mathcal{H}_{i j k} v^{k}, \quad \mathcal{H}^{* i j}=\mathcal{H}^{* i j k} v_{k}, \quad v_{i}=\frac{\partial_{i} a}{\sqrt{g^{j k} \partial_{j} a \partial_{k} a}}, \\
\mathcal{H} & =H+\mathcal{C}_{3}, \quad \mathcal{H}^{* i j k}=\frac{1}{3 ! \sqrt{s \operatorname{det} g}} \epsilon^{i j k l m n} \mathcal{H}_{l m n}, \quad H=d B
\end{aligned}
$$

and

$$
\begin{aligned}
d \mathcal{L}_{\mathrm{WZ}} & =h_{7}, \quad h_{7}=h^{(7)}-\frac{c}{2} h^{(4)} \mathcal{H}, \\
h^{(4)} & =c\left[\frac{1}{2} \mathbf{L}^{A} \mathbf{L}^{B} \bar{L} \Gamma_{A B} L-\frac{6 \lambda}{4 !} \epsilon_{a_{1} \cdots a_{4}} \mathbf{L}^{a_{1}} \cdots \mathbf{L}^{a_{4}}\right], \\
h^{(7)} & =c^{2}\left[\frac{1}{5 !} \mathbf{L}^{A_{1}} \cdots \mathbf{L}^{A_{5}} \bar{L} \Gamma_{A_{1} \cdots A_{5}} L-\frac{6 \lambda}{7 !} \epsilon_{a_{1}^{\prime} \cdots a_{7}^{\prime}} \mathbf{L}^{a_{1}^{\prime}} \cdots \mathbf{L}^{a_{7}^{\prime}}\right], \\
-c d \mathcal{H} & =h^{(4)}
\end{aligned}
$$

with $c^{2}=i \sqrt{s}$ for the $\kappa$-invariance of the action.

Observe that under the expansion (C.3),

$$
\begin{aligned}
& a=a_{p p}, \quad v_{i}=\Lambda v_{i}^{p p}+O\left(\Lambda^{3}\right), \quad \mathcal{H}_{i j k}=\Lambda^{3} \mathcal{H}_{i j k}^{p p}+O\left(\Lambda^{5}\right), \quad \mathcal{H}_{i j}=\Lambda^{2} \mathcal{H}_{i j}^{p p}+O\left(\Lambda^{4}\right), \\
& \mathcal{H}^{* i j k}=\Lambda^{-3} \mathcal{H}_{p p}^{* i j k}+O\left(\Lambda^{-1}\right), \quad \mathcal{H}_{i j}^{*}=\Lambda^{2} \mathcal{H}_{i j}^{* p p}+O\left(\Lambda^{4}\right)
\end{aligned}
$$


where we assume that $H=\Lambda^{3} H_{p p}$. These imply that

$$
\begin{aligned}
& \mathcal{L}_{\mathrm{PST}}=\Lambda^{6} \mathcal{L}_{\mathrm{PST}}^{p p}+O\left(\Lambda^{8}\right), \\
& \mathcal{L}_{\mathrm{PST}}^{p p}=\sqrt{s \operatorname{det}\left(g_{i j}^{p p}-i c^{2} \mathcal{H}_{i j}^{* p p}\right)}+c^{2} \frac{\sqrt{s \operatorname{det} g_{p p}}}{4} \mathcal{H}_{p p}^{* i j} \mathcal{H}_{i j}^{p p} .
\end{aligned}
$$

The WZ term $h_{7}$ is expanded as

$$
\begin{aligned}
h_{7} & =\Lambda^{6} h_{7}^{p p}, \quad h_{7}^{p p}=h_{p p}^{(7)}-\frac{c}{2} h_{p p}^{(4)} \mathcal{H}_{p p} \\
h_{p p}^{(7)} & =c^{2}\left[\frac{1}{5 !} \mathbf{L}^{A_{1}} \cdots \mathbf{L}^{A_{5}} \bar{L} \Gamma_{A_{1} \cdots A_{5}} L-\frac{6 \lambda}{\sqrt{2}} \mathbf{L}^{-} \mathbf{L}^{4} \cdots \mathbf{L}^{9}\right] \\
h_{p p}^{(4)} & =c\left[\frac{1}{2} \mathbf{L}^{A} \mathbf{L}^{B} \bar{L} \Gamma_{A B} L+\frac{6 \lambda}{\sqrt{2}} \mathbf{L}^{-} \mathbf{L}^{1} \mathbf{L}^{2} \mathbf{L}^{3} \mathbf{L}^{4}\right] .
\end{aligned}
$$

The $\Lambda^{6}$ factor is absorbed into the definition of the tension as $T=\Lambda^{-6} T_{p p}$. The pp-wave M5-brane action is given as

$$
S=T_{p p} \int \mathcal{L}_{\mathrm{PST}}^{p p}+\mathcal{L}_{W Z}^{p p}
$$

with (C.32) and $h_{7}^{p p}=d \mathcal{L}_{W Z}^{p p}$ with (C.33).

Following the procedure explained in section 8.1, one can show that the $(p+2)$-form

$h_{p+2}^{p p}$ is a non-trivial element of the CE cohomology. The $(p+1)$-dimensional form of the WZ term can be obtained easily as was done in section 8.2.

\section{References}

[1] J. M. Maldacena, "The large N limit of superconformal field theories and supergravity," Adv. Theor. Math. Phys. 2 (1998) 231 [Int. J. Theor. Phys. 38 (1999) 1113] arXiv:hep-th/9711200; S. S. Gubser, I. R. Klebanov and A. M. Polyakov, "Gauge theory correlators from non-critical string theory," Phys. Lett. B 428 (1998) 105 arXiv:hep-th/9802109; E. Witten, "Anti-de Sitter space and holography," Adv. Theor. Math. Phys. 2 (1998) 253 arXiv:hep-th/9802150.

[2] D. Berenstein, J. M. Maldacena and H. Nastase, "Strings in flat space and pp waves from N = 4 super Yang Mills," JHEP 0204, 013 (2002) arXiv:hep-th/0202021.

[3] R. Penrose, "Any spacetime has a plane wave as a limit," Differential geometry and relativity, Reidel, Dordrecht, 1976, pp. 271-275; 
R. Gueven, "Plane wave limits and T-duality," Phys. Lett. B 482 (2000) 255 arXiv:hep-th/0005061;

M. Blau, J. Figueroa-O'Farrill, C. Hull and G. Papadopoulos, "Penrose limits and maximal supersymmetry," Class. Quant. Grav. 19 (2002) L87 arXiv:hep-th/0201081.

[4] E. Inönü and E. P. Wigner, Proc. Natl. Acad. Sci. U.S.A. 39 (1953) 510;

E. Inönü, in "Group Theoretical Concepts and Methods in Elementary Particle Physics" ed. F. Gursey (Gordon and Breach, New York, 1964).

[5] M. Hatsuda, K. Kamimura and M. Sakaguchi, "From super- $\mathrm{AdS}_{5} \times \mathrm{S}^{5}$ algebra to super-pp-wave algebra," Nucl. Phys. B 632 (2002) 114 arXiv:hep-th/0202190.

[6] M. Hatsuda, K. Kamimura and M. Sakaguchi, "Super-PP-wave algebra from super-AdS $\times \mathrm{S}$ algebras in eleven-dimensions," Nucl. Phys. B 637 (2002) 168 arXiv:hep-th/0204002.

[7] J. Gomis and H. Ooguri, "Non-relativistic closed string theory," J. Math. Phys. 42, 3127 (2001) arXiv:hep-th/0009181.

[8] U. H. Danielsson, A. Guijosa and M. Kruczenski, "IIA/B, wound and wrapped," JHEP 0010, 020 (2000) arXiv:hep-th/0009182.

[9] J. A. Garcia, A. Guijosa and J. D. Vergara, "A membrane action for OM theory," Nucl. Phys. B 630, 178 (2002) arXiv:hep-th/0201140.

[10] J. Brugues, T. Curtright, J. Gomis and L. Mezincescu, "Non-relativistic strings and branes as non-linear realizations of Galilei groups," Phys. Lett. B 594, 227 (2004) arXiv:hep-th/0404175

[11] J. Gomis, K. Kamimura and P. K. Townsend, "Non-relativistic superbranes," JHEP 0411, 051 (2004) arXiv:hep-th/0409219.

[12] J. Gomis, F. Passerini, T. Ramirez and A. Van Proeyen, "Non relativistic Dp-branes," JHEP 0510 (2005) 007 arXiv:hep-th/0507135.

[13] K. Kamimura and T. Ramirez, "Brane dualities in non-relativistic limit," arXiv:hep-th/0512146. 
[14] J. Brugues, J. Gomis and K. Kamimura, "Newton-Hooke algebras, non-relativistic branes and generalized pp-wave metrics," arXiv:hep-th/0603023.

[15] J. Gomis, J. Gomis and K. Kamimura, "Non-relativistic superstrings: A new soluble sector of $\mathrm{AdS}_{5} \times \mathrm{S}^{5}, "$ JHEP 0512 (2005) 024 arXiv:hep-th/0507036.

[16] M. Sakaguchi and K. Yoshida, "D-branes of covariant AdS superstrings," Nucl. Phys. B 684 (2004) 100 arXiv:hep-th/0310228.

[17] M. Sakaguchi and K. Yoshida, "Notes on D-branes of type IIB string on $\mathrm{AdS}_{5} \times \mathrm{S}^{5}$," Phys. Lett. B 591 (2004) 318 arXiv:hep-th/0403243.

[18] M. Sakaguchi and K. Yoshida, "D-branes of covariant AdS superstrings: An overview," arXiv:hep-th/0408208.

[19] M. Sakaguchi and K. Yoshida, "Noncommutative D-branes from Covariant AdS Superstring," OIQP-06-03, KEK-TH-1076.

[20] J. A. De Azcarraga and P. K. Townsend, "Superspace Geometry And Classification Of Supersymmetric Extended Objects," Phys. Rev. Lett. 62 (1989) 2579.

[21] C. Chevalley and S. Eilenberg, Trans. Am. Math. Soc. 63 (1948) 85.

[22] C. Chryssomalakos, J. A. de Azcarraga, J. M. Izquierdo and J. C. Perez Bueno, "The geometry of branes and extended superspaces," Nucl. Phys. B 567 (2000) 293 arXiv:hep-th/9904137.

[23] M. Sakaguchi, "IIB-branes and new space-time superalgebras," JHEP 0004 (2000) 019 arXiv:hep-th/9909143.

[24] M. Hatsuda and M. Sakaguchi, "Wess-Zumino term for AdS superstring," Phys. Rev. D 66 (2002) 045020 arXiv:hep-th/0205092.

[25] M. Hatsuda, K. Kamimura and M. Sakaguchi, "Gauge invariant action for superstring in Ramond-Ramond plane-wave background," Nucl. Phys. B 644 (2002) 40 arXiv:hep-th/0207157.

[26] M. Sakaguchi and K. Yoshida, "Dirichlet branes of the covariant open supermembrane in $\mathrm{AdS}_{4} \times \mathrm{S}^{7}$ and $\mathrm{AdS}_{7} \times \mathrm{S}^{4}, "$ Nucl. Phys. B 681 (2004) 137 arXiv:hep-th/0310035. 
[27] M. Sakaguchi and K. Yoshida, "Open M-branes on $\mathrm{AdS}_{4 / 7} \times \mathrm{S}^{7 / 4}$ revisited," Nucl. Phys. B 714 (2005) 51 arXiv:hep-th/0405109.

[28] G. W. Gibbons and C. E. Patricot, "Newton-Hooke space-times, Hpp-waves and the cosmological constant," Class. Quant. Grav. 20 (2003) 5225 arXiv:hep-th/0308200.

[29] K. Skenderis and M. Taylor, "Branes in AdS and pp-wave spacetimes," JHEP 0206 (2002) 025 [arXive:hep-th/0204054].

[30] P. Bain, K. Peeters and M. Zamaklar, "D-branes in a plane wave from covariant open strings," Phys. Rev. D 67 (2003) 066001 arXiv:hep-th/0208038.

[31] P. Bain, P. Meessen and M. Zamaklar, "Supergravity solutions for D-branes in Hppwave backgrounds," Class. Quant. Grav. 20 (2003) 913 arXiv:hep-th/0205106.

[32] A. Dabholkar and S. Parvizi, "Dp branes in pp-wave background," Nucl. Phys. B 641 (2002) 223 arXiv:hep-th/0203231.

[33] M. Billo and I. Pesando, "Boundary states for GS superstrings in an Hpp wave background," Phys. Lett. B 536 (2002) 121 arXiv:hep-th/0203028.

[34] O. Bergman, M. R. Gaberdiel and M. B. Green, "D-brane interactions in type IIB plane-wave background," JHEP 0303 (2003) 002 arXiv:hep-th/0205183;

M. R. Gaberdiel and M. B. Green, "The D-instanton and other supersymmetric D-branes in IIB plane-wave string theory," Annals Phys. 307 (2003) 147 arXiv:hep-th/0211122.

[35] K. Skenderis and M. Taylor, "Open strings in the plane wave background. I: Quantization and symmetries," Nucl. Phys. B 665 (2003) 3 arXiv:hep-th/0211011;

K. Skenderis and M. Taylor, "Open strings in the plane wave background. II: Superalgebras and spectra," JHEP 0307 (2003) 006 arXiv:hep-th/0212184;

K. Skenderis and M. Taylor, "An overview of branes in the plane wave background," Class. Quant. Grav. 20 (2003) S567 arXiv:hep-th/0301221;

D. Z. Freedman, K. Skenderis and M. Taylor, "Worldvolume supersymmetries for branes in plane waves," arXiv:hep-th/0306046.

[36] M. Cederwall, A. von Gussich, B. E. W. Nilsson, P. Sundell and A. Westerberg, "The Dirichlet super-p-branes in ten-dimensional type IIA and IIB supergravity," 
Nucl. Phys. B 490 (1997) 179 arXiv:hep-th/9611159;

E. Bergshoeff and P. K. Townsend, "Super D-branes," Nucl. Phys. B 490 (1997) 145 arXiv:hep-th/9611173.

[37] M. Aganagic, C. Popescu and J. H. Schwarz, "D-brane actions with local kappa symmetry," Phys. Lett. B 393 (1997) 311 arXiv:hep-th/9610249;

M. Aganagic, C. Popescu and J. H. Schwarz, "Gauge-invariant and gauge-fixed Dbrane actions," Nucl. Phys. B 495 (1997) 99 arXiv:hep-th/9612080;

[38] M. Hatsuda and K. Kamimura, "Wess-Zumino terms for AdS D-branes," Nucl. Phys. B 703 (2004) 277 arXiv:hep-th/0405202.

[39] R. Roiban and W. Siegel, "Superstrings on $\operatorname{AdS}_{5} \times \mathrm{S}^{5}$ supertwistor space," JHEP 0011 (2000) 024 arXiv:hep-th/0010104.

[40] R. R. Metsaev and A. A. Tseytlin, "Type IIB superstring action in $\operatorname{AdS}_{5} \times \mathrm{S}^{5}$ background," Nucl. Phys. B 533 (1998) 109 arXiv:hep-th/9805028.

[41] R. R. Metsaev and A. A. Tseytlin, "Supersymmetric D3 brane action in $\mathrm{AdS}_{5} \times \mathrm{S}^{5}$," Phys. Lett. B 436 (1998) 281 arXiv:hep-th/9806095.

[42] N. Kim and J. T. Yee, "Supersymmetry and branes in M-theory plane-waves," Phys. Rev. D 67 (2003) 046004 arXiv:hep-th/0211029.

[43] M. Hatsuda and M. Sakaguchi, "Wess-Zumino term for the AdS superstring and generalized Inönü-Wigner contraction," Prog. Theor. Phys. 109 (2003) 853 arXiv:hep-th/0106114.

[44] J. A. de Azcarraga, J. M. Izquierdo, M. Picon and O. Varela, "Generating Lie and gauge free differential (super)algebras by expanding Maurer-Cartan forms and ChernSimons supergravity," Nucl. Phys. B 662 (2003) 185 arXiv:hep-th/0212347.

[45] J. A. de Azcarraga, J. M. Izquierdo, M. Picon and O. Varela, "Extensions, expansions, Lie algebra cohomology and enlarged superspaces," Class. Quant. Grav. 21 (2004) S1375 arXiv:hep-th/0401033.

[46] K. Sugiyama and K. Yoshida, "Supermembrane on the pp-wave background," Nucl. Phys. B 644 (2002) 113 arXiv:hep-th/0206070. 
[47] M. Sakaguchi and K. Yoshida, "Dirichlet branes of the covariant open supermembrane on a pp-wave background," Nucl. Phys. B 676 (2004) 311 arXiv:hep-th/0306213.

[48] E. Bergshoeff, E. Sezgin and P. K. Townsend, "Supermembranes And ElevenDimensional Supergravity," Phys. Lett. B 189 (1987) 75;

E. Bergshoeff, E. Sezgin and P. K. Townsend, "Properties Of The Eleven-Dimensional Super Membrane Theory," Annals Phys. 185 (1988) 330.

[49] P. Pasti, D. P. Sorokin and M. Tonin, "Covariant action for a D = 11 five-brane with the chiral field," Phys. Lett. B 398 (1997) 41 arXiv:hep-th/9701037;

I. A. Bandos, K. Lechner, A. Nurmagambetov, P. Pasti, D. P. Sorokin and M. Tonin, "Covariant action for the super-five-brane of M-theory," Phys. Rev. Lett. 78 (1997) 4332 arXiv:hep-th/9701149.

[50] B. de Wit, K. Peeters, J. Plefka and A. Sevrin, "The M-theory two-brane in $\mathrm{AdS}_{4} \times \mathrm{S}^{7}$ and $\mathrm{AdS}_{7} \times \mathrm{S}^{4}, "$ Phys. Lett. B 443 (1998) 153 arXiv:hep-th/9808052.

[51] P. Claus, "Super M-brane actions in $\mathrm{AdS}_{4} \times \mathrm{S}^{7}$ and $\mathrm{AdS}_{7} \times \mathrm{S}^{4}$, " Phys. Rev. D 59 (1999) 066003 arXiv:hep-th/9809045.

[52] P. Claus, R. Kallosh and A. Van Proeyen, "M 5-brane and superconformal $(0,2)$ tensor multiplet in 6 dimensions," Nucl. Phys. B 518 (1998) 117 arXiv:hep-th/9711161.

[53] R. R. Metsaev, "Type IIB Green-Schwarz superstring in plane wave RamondRamond background," Nucl. Phys. B 625 (2002) 70 arXiv:hep-th/0112044.

[54] R. R. Metsaev, "Supersymmetric D3 brane and N = 4 SYM actions in plane wave backgrounds," Nucl. Phys. B 655 (2003) 3 arXiv:hep-th/0211178. 